Benchmark solutions

\title{
Formulation of exactly balanced solvers for blood flow in elastic vessels and their application to collapsed states
}

\author{
J. Murillo*, A. Navas-Montilla, P. García-Navarro \\ Fluid Mechanics-LIFTEC, CSIC-Universidad de Zaragoza, Zaragoza, Spain
}

\section{A R T I C L E I N F O}

\section{Article history:}

Received 10 May 2018

Revised 6 March 2019

Accepted 15 April 2019

Available online xxx

\section{Keywords:}

ARoe

HLLS

Positivity preserving

Flow limitation

Extreme collapse

Stability

\begin{abstract}
A B S T R A C T
In this work, numerical solvers based on extensions of the Roe and HLL schemes are adapted to deal with test cases involving extreme collapsing conditions in elastic vessels. To achieve this goal, the system is transformed to provide a conservation-law form, allowing to define Rankine-Hugoniot conditions. The approximate solvers allow to describe the inner states of the solution. Therefore, source term fixes can be used to prevent unphysical values of vessel area and, at the same time, the eigenvalues of the system control stability. Numerical solvers of different order are tested using a wide variety of Riemann problems, including extreme vessel collapse and blockage. In all cases, the robustness of the approximate solvers presented here is checked using first and third order methods in time and space, using the WENO reconstruction scheme in combination with the TVDRK3 method.
\end{abstract}

(C) 2019 Elsevier Ltd. All rights reserved.

\section{Introduction}

Human venous and arterial flow is sensitive to variations in the body positioning. When analysing in detail changes due to the operation of the baroreceptor reflex, gravity-driven flow pressure variations in time-dependent postural maneuvers are of relevance [1]. On the other hand, the mechanical behavior of vessels strongly differs if comparing veins and arteries. Veins exhibit a highly nonlinear pressure-area relation due to their flexibility, and they collapse in sitting or standing humans while arteries do not. The appropriate treatment of all these phenomena requires nontrivial numerical solvers, that must ensure accuracy and stability when dealing with time-dependent postural maneuvers and timedependent pressure variations in coexistence with spatial variations of the mechanical properties of the vessel wall.

Both arterial and venous system can be modeled using one-dimensional (1D) approaches [2-4], for blood flow in large/medium arteries and veins involving mass and momentum conservation, leading to a hyperbolic system of equations with source terms. In the literature 1D formulations in terms of conserved variables [5-12] as well as in terms of primitive variables [13-16] are presented. It has been pointed out that the formulation in primitive variables may result in an inadequate estimation of the shock propagation velocity [17-19].

\footnotetext{
* Corresponding author.

E-mail addresses: Javier.Murillo@unizar.es (J. Murillo), anavas@unizar.es (A. Navas-Montilla), pigar@unizar.es (P. García-Navarro).
}

The numerical discretization of the source terms associated to spatial variation of mechanical and geometrical properties of vessels requires an special care [11,20]. The same conclusion is derived when analyzing other hyperbolic systems such as the 1D shallow water equations (SWE). Both mathematical models include geometric-type source terms that can be expressed as the product of a function of the conserved quantities and a geometric function that depends upon the position and can be discontinuous [22,23].

It is well known that, if the geometric-type source terms are not treated correctly, spurious oscillations may be generated in the numerical solution leading to failure. The correct balance between fluxes and source terms, or well balanced solutions, [21,24] is the first step to correctly reproduce steady solutions in the presence of source terms. It is pertinent to point out that, in presence of source terms, resonant problems can be defined, leading to multiple solutions in the SWE [25]. This phenomena has also been described in collapsible vessels $[17,26]$.

The numerical innovations developed for the SWE, based on well balanced solutions, can be applied to the analysis of the flow in collapsible vessels [6,11]. High-order path-conservative numerical schemes that exactly preserve steady solutions in any flow regime [41] are of interest. They can include variable geometrical properties [20] and are extensible to the resolution of vessel junctions [27].

As the selection of families of paths is not trivial task $[25,28,29]$, it may be desirable to use alternative strategies. In [30], with only a few modifications of existing shock-capturing Total Variation Diminishing (TVD) schemes, an extension of a TVD scheme was pre- 
sented in combination with a Runge-Kutta time integration. The resulting tool provided accurate solutions when compared with those presented in [20]. High order extensions can also be devised by combining a weighted essentially non-oscillatory (WENO) reconstruction of the conserved variable with Runge-Kutta time integrators [9].

Tube laws for thin walled vessels have a sigmoid shape where pressure falls exponentially when the area tends to zero but they never fully close. If using this type of law for modelling flow in veins, when veins collapse the blood flow is greatly reduced and a negative value of transmural pressure is observed. Therefore, classical waves in the conventional Riemann problem cannot be connected to the zero state with a zero value of vessel area [31]. When approaching a collapsed state it is of utmost importance to control the approximations made in the numerical scheme. For the SWE it has been shown that negative non-physical values of crosssectional area must be avoided. Numerical codes crash because the eigenvalues do not determine the time step size as a result of the not pure hyperbolic character of the system of equations in such conditions [32].

The capabilities of numerical methods preserving the well balanced-property can be enhanced to ensure the exactly well balanced property. Exactly well-balanced methods, named commonly energy balanced numerical schemes, also ensure conservation in exact solutions derived from the entropy function of the system, in our case an energy conservation law. A challenging aspect to be faced by energy-balanced schemes is the robustness of the numerical algorithm in resonant cases and in transitions from supercritical to subcritical flow in energy dissipating shocks or elastic jumps [26].

An alternative to path-conservative numerical schemes are the augmented solvers ARoe (Augmented Roe) presented in [33-36] and HLLS [37] used here. Comparisons between pathconservative based schemes, such as the HLLE and DOT solvers, and the ARoe solver are presented in [40] in the framework of the SWE. The ARoe solver was previously applied to ensure a correct treatment of blood flow in arteries with discontinuous mechanical and geometrical properties and variable external pressure in [38] expressed in fluctuation form. The ARoe solver can also be extended to schemes with arbitrary order of accuracy $[22,39]$.

When analyzing the SWE hydrostatic or gravity forces and friction forces have the same effect on the flow as the observed in the governing equations for blood flow in elastic vessels and they can be formulated exactly in the same way. Variations in atmospheric pressure for the SWE or in the external pressure in vessels are equivalent. The advantage in the SWE is that the relation between water depth and pressure is linear, which allows to express the system of equations in conservative form. Variations in bed elevation in channels or changes in vessel elevation provide hydrostatic variations in the pressure and, in consequence, have an analogous impact in the flow. In the context of the SWE, it is well known that well-balancing problems appear when hydrostatic pressure variations associated to variations in bed elevation are not considered in the approximate solution used to construct the numerical solver $[22,33,34,36]$. Therefore, in this work, changes in pressure derived from changes in vessel elevation are unified with the rest of variations of pressure and, as will be seen in the applications, this formulation preserves the exact solution in exactly balanced RP's.

Friction forces in the SWE can cause problems because of stiffness. The current tendency to avoid numerical errors (lack of balance in the solution and/or oscillations) arising from the friction terms is the use of an implicit treatment of the resistance source term. However, even though implicit discretization of the friction terms ensures stability, a detailed analysis shows that it does not provide an exact balance among fluxes and source terms in steady state cases $[42,43]$, leading to undesirable non uniform dis- charge values. Explicit discretizations of the friction can also lead to non uniform discharge values. The upwind unified treatment $[36,43,44]$ of all terms, including boundary shear stress, ensures an exact balance among fluxes and source terms in steady cases with non-zero velocity.

This work focuses on the application of the ARoe and HLLS solvers to flow in collapsible tubes with discontinuous mechanical properties including collapsed states. To the best of our knowledge, there are not previous works focused on the application and design of numerical solvers devoted to the solution of Riemann problems in collapsed vessels. Recently, the presence of collapsed states in the exact solution has been analyzed in [31], remarking the differences between veins and arteries. Another novelty of this work is the use of the HLLS solver. The system of equations can not be expressed using a conservation-law form, and the flux function used to derive the eigenvalues of the system can not be defined. This request can be avoided by defining a suitable Jacobian matrix, as in [38], but the HLLS requires the definition of a flux to define jumps across shocks. The transformation of the initial system of equations presented here provides an equivalent system written in conservation-law form, where a flux function is defined, allowing the application of the HLLS scheme.

This paper is organized as follows. In Section 2 the mathematical model is recalled. In Section 3 the system is transformed to provide a conservation-law form, allowing to define jumps across shocks or Rankine-Hugoniot (RH) conditions necessary to apply the ARoe and HLLS solvers in Section 4 and 5 respectively. For both solvers conditions of vessel collapse are analyzed. Section 6 includes a summary of the steps required to compute the fluxes used for both ARoe and HLLS solvers. The flux solvers are extended from first to third order using the weighted essentially non-oscillatory (WENO) reconstruction scheme in combination with the optimal third-order total variation diminishing Runge-Kutta (TVDRK3) in Section 7. Numerical results in a wide variety of cases including severe collapse are discussed in Section 12.

\section{1D mathematical models in arteries and veins}

Simplified 1D models can be used to represent the essential physical features of wave propagation in compliant vessels. The resulting 1D equations involve cross section average of the mass and momentum that, in conservative form, lead to the following firstorder, nonlinear hyperbolic system:

$$
\begin{aligned}
& \partial_{t} \mathbf{U}+\partial_{\chi} \mathcal{F}(\mathbf{U})=\mathbf{G}_{\chi}, \\
& \text { with } \mathbf{U}=\mathbf{U}(x, t) \text { and } \\
& \mathbf{U}=\left[\begin{array}{l}
A \\
Q
\end{array}\right], \quad \mathcal{F}(\mathbf{U})=\left[\begin{array}{c}
Q \\
\kappa \frac{Q^{2}}{A}
\end{array}\right], \\
& \mathbf{G}_{\chi}=\left[\begin{array}{c}
0 \\
-\frac{A}{\rho} \frac{\partial p}{\partial \chi}-\frac{f}{\rho}-g A \frac{\partial \eta}{\partial \chi}
\end{array}\right]=\left[\begin{array}{c}
0 \\
G_{\chi}
\end{array}\right],
\end{aligned}
$$

where $x$ is the axial coordinate along the vessel, $t$ is the time, $A$ is the cross-sectional area, $Q=A u$ is the volume flow rate with $u$ the cross sectional average axial velocity, $p(x, t)$ is the average internal pressure over the cross section, $f$ is the friction force per unit length, $\rho$ is the blood density and $\eta$ is a coordinate perpendicular to Earth surface, that accounts for the gravitational forces due to the presence of gravity acceleration $g$. The parameter $\kappa$ depends on the assumed velocity profile, and a blunt velocity profile is assumed in this work with $\kappa=1$. If Poiseuille flow is considered for collapsible tubes the friction force becomes $f=8 \pi \mu u$. This system of equations is closed with a pressure-area relation of the form:

$p(x, t)-p_{e}(x, t)=\psi, \quad \psi=\psi\left(K, A, p_{o}, A_{o}, x\right)$, 
where $p_{e}$ is the external pressure and function $\psi$ is the elastic transmural pressure that depends on the vessel stiffness $K=K(x)$. Following [45] transmural pressure is assumed of the form:

$\psi=K \sigma+p_{0}, \quad \sigma=\alpha^{m}-\alpha^{n}$,

with $\alpha=A / A_{0}$. Vessel stiffness $K$ has different formulations for arteries, and exponents in $\sigma$ are of the form $m>0$ and $n \in[-2,0]$. Finally $A_{o}$ is the vessel cross-sectional area for which the transmural pressure $\psi$ is $p_{0}$.

\subsection{Quasilinear form}

From (2) it is possible to define a Jacobian matrix A of the flux $\mathcal{F}(\mathbf{U})$ defined as:

$\mathbf{A}=\frac{\partial \mathcal{F}(\mathbf{U})}{\partial \mathbf{U}}=\left[\begin{array}{cc}0 & 1 \\ -u^{2} & 2 u\end{array}\right]$,

with $u=Q / A$, the flow velocity. Using (4), the pressure derivative can be expanded as follows:

$\partial_{x} p=\partial_{x} p_{e}+\partial_{x} K \sigma+K \sigma_{A} \partial_{x} A+K \sigma_{A_{0}} \partial_{x} A_{0}+\partial_{x} p_{o}$,

with

$$
\begin{aligned}
& \sigma_{A}=\frac{\partial \sigma}{\partial A}=m A^{m-1} A_{0}{ }^{-m}-n A^{n-1} A_{0}{ }^{-n}, \\
& \sigma_{A_{0}}=\frac{\partial \sigma}{\partial A_{0}}=-m A^{m} A_{0}{ }^{-(m+1)}+n A^{n} A_{0}{ }^{-(n+1)} .
\end{aligned}
$$

Source terms in $\mathbf{G}_{\chi}$ are expressed as

$$
\mathbf{G}_{\chi}=-\mathbf{B} \frac{\partial \mathbf{U}}{\partial x}+\mathbf{S}_{\chi}, \quad \mathbf{B}=\left[\begin{array}{cc}
0 & 0 \\
c^{2} & 0
\end{array}\right],
$$

where $\mathbf{B}$ accounts for the cross-sectional variations in space, with $c^{2}=\frac{A}{\rho} K \sigma_{A}$, and matrix $\mathbf{S}_{\chi}$ is given by

$\mathbf{S}_{\chi}=\left[\begin{array}{c}0 \\ -\frac{A}{\rho} \frac{\partial p}{\partial x}+c^{2} \frac{\partial A}{\partial x}-\frac{f}{\rho}-g A \frac{\partial \eta}{\partial x}\end{array}\right]$.

Using (5) and (8) the following matrix $\mathbf{J}$ can be built

$\mathbf{J}=\mathbf{A}+\mathbf{B}, \quad \mathbf{J}=\left[\begin{array}{cc}0 & 1 \\ c^{2}-u^{2} & 2 u\end{array}\right]$

Now, system in (1) is written in quasilinear form as follows

$$
\frac{\partial \mathbf{U}}{\partial t}+\mathbf{J}(\mathbf{U}) \frac{\partial \mathbf{U}}{\partial x}=\mathbf{S}_{\chi}
$$

and, depending on the value of parameters $m$ and $n$, system (1) can be considered of hyperbolic nature [17]. Matrix $\mathbf{J}$ has two eigenvalues, $\lambda^{1}=u-c$ and $\lambda^{2}=u+c$, and two real eigenvectors, $\mathbf{e}^{1}=$ $\left(1, \lambda^{1}\right)^{T}$ and $\mathbf{e}^{2}=\left(1, \lambda^{2}\right)^{T}$, appear.

\section{Semi-discrete approximate RP}

Between each two adjacent cells $i$ and $i+1$ of constant length $\Delta x$ a local RP posed for a time step $t \in(0, \Delta t)$ is defined for the system as follows:

$$
\frac{\partial \hat{\mathbf{U}}}{\partial t}+\frac{\partial \mathcal{F}(\hat{\mathbf{U}})}{\partial x}=\mathbf{G}_{\chi}, \quad \hat{\mathbf{U}}(x, 0)=\left\{\begin{array}{ccc}
\mathbf{U}_{i} & \text { if } & x<0 \\
\mathbf{U}_{i+1} & \text { if } & x>0
\end{array}\right.
$$

in the control volume represented in Fig. 1, where $\hat{\mathbf{U}}$ is an approximate solution of (12) and $\mathbf{U}_{i}=\mathbf{U}_{i}^{n}$ and $\mathbf{U}_{i+1}=\mathbf{U}_{i+1}^{n}$ are the initial conditions for $\hat{\mathbf{U}}$ at time $t=0$ at the left and right side of the RP respectively, with

$\mathbf{U}_{i}^{n}=\frac{1}{\Delta x} \int_{0}^{\Delta x} \mathbf{U}(x, t=0) d x$.

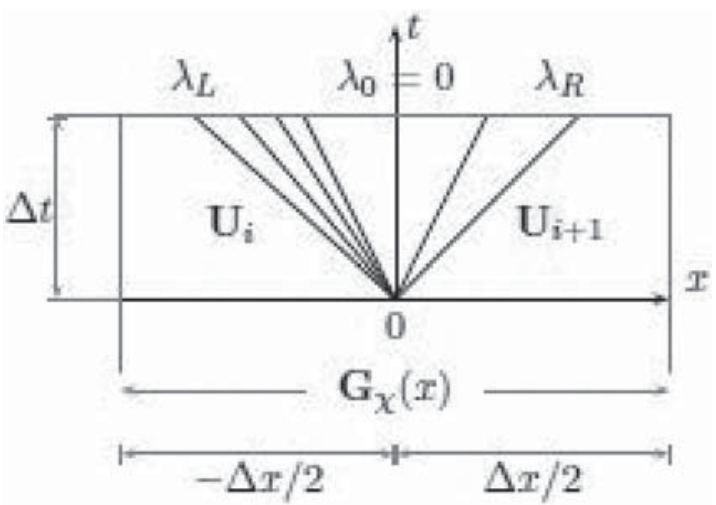

Fig. 1. Integration control volume defined by a time interval $[0, \Delta t]$ and a space interval $[-\Delta x / 2, \Delta x / 2]$.

The RP in (12) will be solved using approximate linear solutions of an initial value problem by means of an explicit conservative formula that, according to the Godunov first order method, is written as:

$\mathbf{U}_{i}^{n+1}=\mathbf{U}_{i}^{n}-\frac{\Delta t}{\Delta x}\left[\mathbf{F}_{i+\frac{1}{2}}^{-}-\mathbf{F}_{i-\frac{1}{2}}^{+}\right]$,

with $\mathbf{F}_{i-1 / 2}^{+}$the numerical fluxes yet to be defined, that will include both the physical flux $\mathcal{F}$ and the source term $\mathbf{G}$, that is, $\mathbf{F}=\mathbf{F}(\mathcal{F}, \mathbf{G})$. The necessary conditions required to define numerical flux $\mathbf{F}$ are presented in this section.

Analogously, Eq. (14) can be rewritten in terms of fluctuations, denoted here by $\delta \mathbf{M}$, leading to

$\mathbf{U}_{i}^{n+1}=\mathbf{U}_{i}^{n}-\frac{\Delta t}{\Delta x}\left[\delta \mathbf{M}_{i+\frac{1}{2}}^{-}+\delta \mathbf{M}_{i-1 / 2}^{+}\right]$,

following the quasi-steady wave-propagation algorithm [46], with,

$\mathbf{F}_{i+\frac{1}{2}}^{-}=\mathcal{F}_{i}+\delta \mathbf{M}_{i-1 / 2}^{+}, \quad \mathbf{F}_{i-\frac{1}{2}}^{+}=\mathcal{F}_{i}-\delta \mathbf{M}_{i+\frac{1}{2}}^{-}$

where instead of defining a numerical flux at each interface $\mathbf{F}$, the solution is explored using differences between the conserved variables and fluxes across the interface. In exactly balanced cases of equilibrium the desired solution will be $\delta \mathbf{M}_{i+1 / 2}^{ \pm}=0$.

It must be noticed that the differential form of the governing equations for 1D blood flow only allows to formulate a flux function for the system in the homogeneous case with constant vessel properties [17], otherwise they cannot be described using a conservation-law form. As a consequence, when managing realistic simulations involving geometrical and mechanical properties, the path-conservative numerical schemes in [20,41], the shockcapturing TVD scheme in [30] or the ARoe solver in [38], were expressed in fluctuation form. This section is devoted to the construction of an approximate RP in conservation-law form, that will allow us to locally define a flux $\mathbf{F}$ in (14).

With independence of the approximate solver used, the Consistency Condition must be satisfied [47]. The control volume is limited by the maximum and minimum wave celerities of the system, $-\Delta x / 2 \leq \lambda_{L} \Delta t$ and $\Delta x / 2 \geq \lambda_{R} \Delta t$, with $\lambda_{L} \geq \lambda_{R}$. Integrating (12) over the control volume the resulting condition is

$$
\begin{aligned}
& \frac{1}{\Delta x \Delta t} \int_{-\frac{\Delta x}{2}}^{\frac{\Delta x}{2}} \hat{\mathbf{U}}(x, \Delta t) d x=\frac{\left(\mathbf{U}_{i+1}+\mathbf{U}_{i}\right)}{2 \Delta t}-\frac{\delta \mathcal{F}_{i+\frac{1}{2}}}{\Delta x} \\
& +\frac{1}{\Delta x \Delta t} \int_{-\frac{\Delta x}{2}}^{\frac{\Delta x}{2}} \int_{0}^{\Delta t} \mathbf{G}_{\chi} d x d t,
\end{aligned}
$$

where $\delta$ symbol is used to express space difference, $\delta(\cdot)_{i+1 / 2}=$ $(\cdot)_{i+1}-(\cdot)_{i}$. The flux difference $\delta \mathcal{F}_{i+\frac{1}{2}}$ is written using an approxi- 
where matrix $\tilde{\mathbf{B}}$ includes an energy balanced approach in the wave

mate Jacobian Ã:

$\delta \mathcal{F}_{i+\frac{1}{2}}=\tilde{\mathbf{A}}_{i+\frac{1}{2}} \delta \mathbf{U}_{i+\frac{1}{2}}, \quad \widetilde{\mathbf{A}}_{i+1 / 2}=\left(\begin{array}{cc}0 & 1 \\ -\widetilde{u}^{2} & 2 \widetilde{u}\end{array}\right)_{i+1 / 2}$,

involving the Roe average value:

$\tilde{u}_{i+1 / 2}=\frac{u_{i} \sqrt{A_{i}}+u_{i+1} \sqrt{A_{i+1}}}{\sqrt{A_{i}}+\sqrt{A_{i+1}}}$.

The source term is included in the Riemann solver as a singular source at the discontinuity point $x=0$ [33]. Considering that source terms are not necessarily constant in time, the following time linearization is applied $[33,34]$

$$
\begin{aligned}
\mathbf{G}_{i+1 / 2} & =\frac{1}{\Delta t} \int_{-\Delta x / 2}^{\Delta x / 2} \int_{0}^{\Delta t} \mathbf{G}_{\chi} d x d t \\
& \approx\left[-\frac{\mathcal{A}}{\rho}(\delta p+g \rho \delta \eta)-\frac{\tilde{f}}{\rho} \Delta x\right]_{i+1 / 2},
\end{aligned}
$$

with $\mathcal{A}$ a suitable value of vessel area that will be defined depending on the flow conditions and $\tilde{f}$ the integral of the friction force in the control volume. The numerical approximation in (20), based on preserving the jump in pressure, will prove successful when seeking energy balanced solutions in Section 3.2.

The pressure variation $\delta p$ can be defined exactly as:

$\delta p_{i+\frac{1}{2}}=\delta\left(p_{e}\right)_{i+\frac{1}{2}}+\delta(K \sigma)_{i+\frac{1}{2}}+\delta\left(p_{0}\right)_{i+\frac{1}{2}}$,

or in terms of the variations of the vessel stiffness $K$ and of the function $\sigma$ using the average values

$\delta(K \sigma)_{i+1 / 2}=(\bar{K} \delta \sigma+\bar{\sigma} \delta K)_{i+1 / 2}$ edge

The variations in the dimensionless function $\sigma$ at each $i+\frac{1}{2}$

$\delta \sigma=\delta\left(A^{m} A_{o}^{-m}\right)-\delta\left(A^{n} A_{o}^{-n}\right)$

can be again expressed in terms of variations in cross sectional areas $A$ and $A_{0}$, using

$\delta\left(A^{m} A_{o}^{-m}\right)=\overline{A^{m}} \delta A_{o}^{-m}+\overline{A_{o}^{-m}} \delta A^{m}, \quad \delta\left(A^{n} A_{o}^{-n}\right)=\overline{A^{n}} \delta A_{o}^{-n}+\overline{A_{o}^{-n}} \delta A^{n}$.

Power functions of the cross sectional areas in (24) are linearized by suitable average values in the following way:

$\delta A^{m}=\widetilde{A^{m-1}} \delta A, \quad \delta A^{n}=\widetilde{A^{n-1}} \delta A, \quad \delta A_{o}^{-m}=\widetilde{A_{o}^{-(m+1)}} \delta A_{o}$,

$\delta A_{o}^{-n}=\widetilde{A_{o}^{-(n+1)}} \delta A_{0}$.

Average values $\widetilde{A^{m-1}}, \widetilde{A^{n-1}}, \widetilde{A_{o}^{-(m+1)}}$ and $\widetilde{A_{o}^{-(n+1)}}$ are functions of $A_{i}$ and $A_{i+1}$ and are defined in Appendix A. With these expansions it is possible to express the pressure variation in (21) as

$\delta p=\delta p_{e}+\bar{\sigma} \delta K+\delta p_{o}+\bar{K} \widetilde{\sigma_{A}} \delta A+\bar{K} \widetilde{\sigma_{A_{0}}} \delta A_{o}$,

with

$\widetilde{\sigma_{A}}=\left[\overline{A_{o}^{-m}} \widetilde{A^{m-1}}-\overline{A_{o}^{-n}} \widetilde{A^{n-1}}\right], \quad \widetilde{{A_{0}}_{0}}=\left[\overline{A^{m}} \widetilde{A_{o}^{-(m+1)}}-\overline{A^{n}} \widetilde{A_{o}^{-(n+1)}}\right]$.

Now the source term $\mathbf{G}_{i+1 / 2}$ can be expressed as

$\mathbf{G}_{i+1 / 2}=-\tilde{\mathbf{B}}_{i+1 / 2} \delta \mathbf{U}_{i+1 / 2}+\mathbf{S}_{i+1 / 2}$, speed estimation:

$\tilde{\mathbf{B}}_{2, i+1 / 2}=\left[\begin{array}{cc}0 & 0 \\ \widetilde{c}^{2} & 0\end{array}\right]_{i+1 / 2}, \quad \widetilde{c}^{2}=\frac{\mathcal{A}}{\rho} \bar{K} \widetilde{\sigma_{A}}$.

and source term $\mathbf{S}_{i+1 / 2}$ can be defined in terms of variations as:

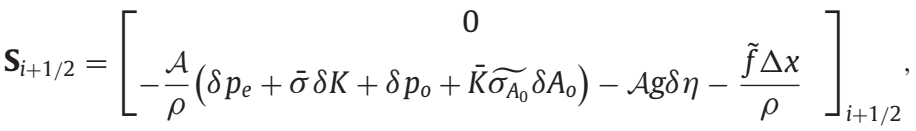

or involving the wave speed estimation $\widetilde{c}$ and the jump in driving pressure $\delta p_{d, i+1 / 2}$

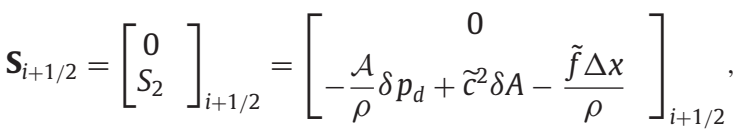

with $p_{d}=p+g \rho \eta$ an equivalent pressure generated by the combination of the pressure and the gravitational force. Now that all terms are redefined, the integral of the solution in the volume of interest is given by

$\int_{-\Delta x / 2}^{+\Delta x / 2} \mathbf{U}(x, \Delta t) d x=\frac{\Delta x}{2}\left(\mathbf{U}_{i}+\mathbf{U}_{i+1}\right)-\delta \mathbf{M}_{i+1 / 2} \Delta t$,

with

$\delta \mathbf{M}_{i+\frac{1}{2}}=(\delta \mathcal{F}-\mathbf{G})_{i+\frac{1}{2}}=(\tilde{\mathbf{A}}+\tilde{\mathbf{B}})_{i+\frac{1}{2}} \delta \mathbf{U}_{i+\frac{1}{2}}-\mathbf{S}_{i+\frac{1}{2}}$.

\subsection{The linear semi-discrete approximate $R P$}

The RP in (12) is now approximated by using the following constant coefficient linear RP [48]

$\frac{\partial \hat{\mathbf{U}}}{\partial t}+\widetilde{\mathbf{J}}_{i+\frac{1}{2}} \frac{\partial \hat{\mathbf{U}}}{\partial x}=\frac{1}{\Delta x} \mathbf{S}_{i+\frac{1}{2}}, \quad \hat{\mathbf{U}}(x, 0)=\left\{\begin{array}{ccc}\mathbf{U}_{i} & \text { if } & x<0 \\ \mathbf{U}_{i+1} & \text { if } & x>0\end{array}\right.$

where $\tilde{\mathbf{J}}_{i+\frac{1}{2}}=\widetilde{\mathbf{J}}_{i+\frac{1}{2}}\left(\mathbf{U}_{i}, \mathbf{U}_{i+1}\right)$ is a constant matrix. Integrating (34) over the same control volume, the following constraint appears

$(\tilde{\mathbf{A}}+\tilde{\mathbf{B}})_{i+\frac{1}{2}} \delta \mathbf{U}_{i+\frac{1}{2}}=\tilde{\mathbf{J}}_{i+\frac{1}{2}} \delta \mathbf{U}_{i+\frac{1}{2}}$,

that is, $\tilde{\mathbf{J}}_{i+\frac{1}{2}}=(\tilde{\mathbf{A}}+\tilde{\mathbf{B}})_{i+\frac{1}{2}}$. Equilibrium in (33) is then also expressed as:

$\delta \mathbf{U}_{i+\frac{1}{2}}=\widetilde{\mathbf{J}}_{i+\frac{1}{2}}^{-1} \mathbf{S}_{i+\frac{1}{2}}$.

The approximate Jacobian $\tilde{\mathbf{J}}$ provides a set of two real eigenvalues $\widetilde{\lambda}^{1}=\widetilde{u}-\widetilde{c}$ and $\widetilde{\lambda}^{2}=\widetilde{u}+\widetilde{c}$ with $\widetilde{\lambda}_{i+\frac{1}{2}}^{1}<\widetilde{\lambda}_{i+\frac{1}{2}}^{2}$, and two eigenvectors $\widetilde{\mathbf{e}}_{i+1 / 2}^{1,2}=\left(1, \tilde{\lambda}^{1,2}\right)_{i+1 / 2}^{T}$. With them it is possible to define two approximate matrices $\widetilde{\mathbf{P}}=\left(\widetilde{\mathbf{e}}^{1}, \widetilde{\mathbf{e}}^{2}\right)$ and $\widetilde{\mathbf{P}}^{-1}$ that diagonalize $\widetilde{\mathbf{J}}$ :

$\widetilde{\mathbf{P}}_{i+1 / 2}^{-1} \widetilde{\mathbf{J}}_{i+1 / 2} \widetilde{\mathbf{P}}_{i+1 / 2}=\tilde{\boldsymbol{\Lambda}}_{i+1 / 2}, \quad \widetilde{\boldsymbol{\Lambda}}_{i+1 / 2}=\left(\begin{array}{cc}\tilde{\lambda}^{1} & 0 \\ 0 & \tilde{\lambda}^{2}\end{array}\right)_{i+1 / 2}$.

Formulation in (34) allows us to define a new flux function of interest,

$\mathbf{F}_{i+1 / 2}\left(\mathbf{U}_{i}\right)=\mathcal{F}\left(\mathbf{U}_{i}\right)+\widetilde{\mathbf{B}}_{i+\frac{1}{2}} \mathbf{U}_{i}=\left(\begin{array}{c}A_{i} u_{i} \\ \widetilde{c}_{i+1 / 2}^{2} A_{i}+A_{i} u_{i}^{2}\end{array}\right)$,

as

$$
\begin{aligned}
\delta \mathbf{F}_{i+1 / 2} & =\mathbf{F}_{i+1 / 2}\left(\mathbf{U}_{i+1}\right)-\mathbf{F}_{i+1 / 2}\left(\mathbf{U}_{i+1}\right) \\
& =\delta \mathcal{F}_{i+1 / 2}+\widetilde{\mathbf{B}}_{i+\frac{1}{2}} \delta \mathbf{U}_{i+1 / 2}=\widetilde{\mathbf{J}}_{i+1 / 2} \delta \mathbf{U}_{i+1 / 2} .
\end{aligned}
$$


Although the new function $\mathbf{F}$ is not a real physical flux it allows to define the following approximate semi-discrete RP in conservation-law form:

$$
\frac{\partial \hat{\mathbf{U}}}{\partial t}+\frac{\delta \mathbf{F}_{i+\frac{1}{2}}(\hat{\mathbf{U}})}{\Delta x}=\frac{1}{\Delta x} \mathbf{S}_{i+\frac{1}{2}}, \quad \hat{\mathbf{U}}(x, 0)=\left\{\begin{array}{ccc}
\mathbf{U}_{i} & \text { if } & x<0 \\
\mathbf{U}_{i+1} & \text { if } & x>0
\end{array},\right.
$$

with the same initial conditions, transforming the result in (33) in

$\delta \mathbf{M}_{i+\frac{1}{2}}=\delta \mathbf{F}_{i+\frac{1}{2}}-\overline{\mathbf{S}}_{i+\frac{1}{2}}$,

involving a flux difference and a source term.

\subsection{Discrete equilibrium and steady state solutions.}

At this point, with independence of the flux solver selected, the source term linearization in (20) ensures an exact integration in steady conditions when $\delta \mathbf{M}_{i+1 / 2}=0$. In this case, (33) results in an equilibrium between a flux difference and a source term:

$\delta \mathcal{F}_{i+\frac{1}{2}}=\mathbf{G}_{i+\frac{1}{2}}$,

leading to:

$\delta(A u)_{i+\frac{1}{2}}=0, \quad \delta\left(A u^{2}\right)_{i+\frac{1}{2}}=-\left(\frac{\mathcal{A}}{\rho} \delta p_{d}+\frac{\tilde{f}}{\rho} \Delta x\right)_{i+\frac{1}{2}}$

But the steady solutions not only have to satisfy momentum equilibrium, they also must ensure convergence to a physically based solution. Therefore, exactly energy balanced solutions will be considered here. For our system of interest, the entropy function is a combination of the mass and the momentum conservation equations expressed in terms of the primitive variables:

$\frac{\partial u}{\partial t}+\frac{\partial}{\partial x}\left(\frac{u^{2}}{2}+\frac{p_{d}}{\rho}\right)=\Phi$,

where $\Phi=\frac{f}{A \rho}$ stands for the viscous dissipation. If this function is integrated in our volume of interest and steady conditions are assumed, the resulting condition is:

$\delta\left(\frac{u^{2}}{2}+\frac{p_{d}}{\rho}\right)_{i+\frac{1}{2}}=-\left(\frac{\tilde{f}}{\bar{A} \rho}\right)_{i+\frac{1}{2}} \Delta x$.

If the equivalent area $\mathcal{A}$ in (20) is approximated by $\mathcal{A}_{E}$ at each $i+\frac{1}{2}$ edge:

$\mathcal{A}_{E}=\bar{A}+\Pi_{E}\left(A_{\min }-\bar{A}\right)$,

where $(:)$ symbol denotes the arithmetic mean, $(\cdot)_{i+1 / 2}=$ $\frac{1}{2}\left[(\cdot)_{i+1}+(\cdot)_{i}\right],(\cdot)_{\min }$ symbol denotes the minimum value, $(\cdot)_{\min }=$ $\min (\cdot)_{i},(\cdot)_{i+1}$. The combination of momentum and energy conservation equations in (43) and (45) leads to the following value of $\Pi_{E}[38]$ :

$\Pi_{E}=\left(\delta\left(A u^{2}\right)-\frac{\bar{A}}{2} \delta u^{2}\right)\left(\frac{\left(\bar{A}-A_{\min }\right) \delta p_{d}}{\rho}\right)^{-1}$,

a dimensionless parameter or weight, limited in this work by $-1 \leq$ $\Pi_{E} \leq 1$.

In cases of static equilibrium, $\Pi_{E}=0$, and the solution reduces to $\delta p_{d}=0$, ensuring the well balanced property. If flow is considered, the equivalent area $\mathcal{A}_{E}$ can be expressed as

$\mathcal{A}_{E}=\frac{1}{2}\left[A_{\min }\left(1+\Pi_{E}\right)+A_{\max }\left(1-\Pi_{E}\right)\right], \quad A_{\min } \leq \mathcal{A}_{E} \leq A_{\max }$,

with $(\cdot)_{\max }=\max (\cdot)_{i},(\cdot)_{i+1}$. Therefore, energy conservation is ensured in steady flow conditions by means of the approximate area $\mathcal{A}_{E}$ that varies linearly with $\Pi_{E}$ between the maximum and minimum values of the vessel area in the discontinuity.

\subsection{Energy dissipation}

The energy balance integral accounting for pressure variations and gravitational effects makes use of the approximation area $\mathcal{A}=$ $\mathcal{A}_{E}$ in (48) limited by the left and right values of cross-sectional area. Using energy conservation arguments it is possible to generate a steady solution resembling that of a converging-diverging nozzle in a flexible vessel, generated by smooth variations of external pressure defined by a bell-shaped curve. Minimum vessel area appears consequently at the point of maximum external pressure equivalent to the nozzle throat. When dealing with nozzles if the flow becomes sonic at this point flow limitation occurs and, downstream this point, a steady shock or jump with a supersonicsubsonic transition where energy is dissipated can be produced. Once sonic blockage is produced, further difference between upstream and downstream pressures is unable to increase the flow across the nozzle throat generated in the vessel [52]. An analogous phenomenon is produced in elastic vessels under some external pressure variations. This phenomenon is observed in the respiratory tract during forced expiration, when the maximal air outflow rate from the lung is independent of the effort put forward by the patient [26].

Therefore, the numerical integration of the source terms must be able to ensure energy balanced solutions but also must be able to dissipate energy and ensure that the maximum flow is independent of the difference between upstream and downstream pressures in sonic blockage conditions.

Depending on the flow conditions and of the speed index $S I=$ $u / c$, the following numerical integration of the source term $S_{2}$ in (31) and selection of pulse wave velocity $\tilde{c}$ are proposed:

- If $u_{i} u_{i+1}>0$

1. In case that a left/right supersonic-subsonic transition appears, $S I_{i}>1, S I_{i+1}<1$ and $A_{i}<A_{i+1}$ or $S I_{i}>-1, S I_{i+1}<-1$ and $A_{i+1}<A_{i}$ respectively, set:

$S_{2}=-\frac{A_{\min }}{\rho} \delta p_{d}+\left(\frac{A_{\min }}{\bar{A}}\right) \widetilde{c}^{2} \delta A, \quad \widetilde{c}^{2}=\frac{\bar{A}}{\rho} \bar{K} \widetilde{\sigma_{A}}$.

2. Otherwise compute the dimensionless parameter $\Pi_{E}$ following (47) and limit it by $-1 \leq \Pi_{E} \leq 1$ if necessary. Then define:

$S_{2}=-\frac{\mathcal{A}_{E}}{\rho} \delta p_{d}+\widetilde{c}^{2} \delta A, \quad \widetilde{c}^{2}=\frac{\mathcal{A}_{E}}{\rho} \bar{K} \widetilde{\sigma_{A}}$.

- If $u_{i} u_{i+1} \leq 0$ impose:

$S_{2}=-\frac{\bar{A}}{\rho} \delta p_{d}+\widetilde{c}^{2} \delta A, \quad \widetilde{c}^{2}=\frac{\bar{A}}{\rho} \bar{K} \widetilde{\sigma_{A}}$.

This procedure summarizes the selection of the pulse wave velocity $\widetilde{c}$ and the source term $S_{2}$ depending on the type of flow transitions.

\section{Augmented roe solver}

According to the Godunov method it is sufficient to provide the solution for $\hat{\mathbf{U}}(x, t)$ at the intercell position $x=0$ in order to derive the updating numerical fluxes in (14). In order to recover the value of the approximate intermediate states $\mathbf{U}_{i}^{-}$and $\mathbf{U}_{i+1}^{+}$at the left and right side of the $(x, t)$ plane solution respectively,

$\mathbf{U}_{i}^{-}=\lim _{x \rightarrow 0^{-}} \hat{\mathbf{U}}_{i}(x, t>0), \quad \mathbf{U}_{i+1}^{+}=\lim _{x \rightarrow 0^{+}} \hat{\mathbf{U}}_{i+1}(x, t>0)$,

the system in (34) is transformed by using $\widetilde{\mathbf{P}}^{-1}$ matrix as follows:

$\widetilde{\mathbf{P}}_{i+\frac{1}{2}}^{-1}\left(\frac{\partial \hat{\mathbf{U}}}{\partial t}+\widetilde{\mathbf{J}}_{i+\frac{1}{2}} \frac{\partial \hat{\mathbf{U}}}{\partial x}\right)=\frac{1}{\Delta x} \widetilde{\mathbf{P}}_{i+\frac{1}{2}}^{-1} \mathbf{S}_{i+\frac{1}{2}}$ 
Expressing (34) in terms of the characteristic variables $\mathbf{V}=$ $\widetilde{\mathbf{P}}_{i+\frac{1}{2}}^{-1} \hat{\mathbf{U}}$, with $\mathbf{V}=\left(V^{1}, V^{2}\right)^{T}$. The following linear RP can be formulated:

$$
\begin{aligned}
& \frac{\partial \mathbf{V}}{\partial t}+\widetilde{\boldsymbol{\Lambda}}_{i+\frac{1}{2}} \frac{\partial \mathbf{V}}{\partial x}=\frac{1}{\Delta x} \mathbf{B}_{i+\frac{1}{2}}^{\beta}, \\
& \mathbf{V}(x, 0)=\left\{\begin{aligned}
\mathbf{V}_{i}=\widetilde{\mathbf{P}}_{i+\frac{1}{2}}^{-1} \mathbf{U}_{i} & \text { if } & x<0 \\
\mathbf{V}_{i+1}=\widetilde{\mathbf{P}}_{i+\frac{1}{2}}^{-1} \mathbf{U}_{i+1} & \text { if } & x>0
\end{aligned}\right.
\end{aligned}
$$

with $\mathbf{B}_{i+\frac{1}{2}}^{\beta}=\left(\widetilde{\mathbf{P}}^{-1} \mathbf{S}\right)_{i+\frac{1}{2}}=\left(\beta^{1}, \beta^{2}\right)_{i+\frac{1}{2}}^{T}$ the set of source strengths defined as:

$\bar{\beta}^{1}=-\frac{S_{2}}{2 \widetilde{c}}, \quad \beta^{2}=-\beta^{1}$.

System in (54) is a decoupled system and each equation

$\frac{\partial V^{r}}{\partial t}+\tilde{\lambda}_{i+\frac{1}{2}}^{r} \frac{\partial V^{r}}{\partial x}=\frac{1}{\Delta x} \beta_{i+\frac{1}{2}}^{r}, \quad r=1,2$

involves the variable $V^{r}$, the wave celery $\tilde{\lambda}^{r}$ and the source strength $\beta_{i+\frac{1}{2}}^{r}$. It is possible to generate a set of independent equations that can be solved exactly for each characteristic variable $V^{r}$. The solution is constructed from the set of wave strengths $\mathbf{A}_{i+\frac{1}{2}}^{\alpha}=$ $\left(\alpha^{1}, \alpha^{2}\right)_{i+\frac{1}{2}}^{T}=\delta \mathbf{V}_{i+\frac{1}{2}}=\widetilde{\mathbf{P}}_{i+\frac{1}{2}}^{-1} \delta \mathbf{U}_{i+\frac{1}{2}}$ with

$\alpha_{i+1 / 2}^{1}=\left(\frac{\tilde{\lambda}^{2} \delta A-\delta Q}{2 \widetilde{c}}\right)_{i+1 / 2}, \quad \alpha_{i+1 / 2}^{2}=-\left(\frac{\tilde{\lambda}^{1} \delta A-\delta Q}{2 \widetilde{c}}\right)_{i+1 / 2}$.

The $\alpha^{r}$ wave strengths allow to express simple linear relations for both conserved variables and flux vector differences as follows:

$$
\delta \mathbf{U}_{i+\frac{1}{2}}=\sum(\alpha \widetilde{\mathbf{e}})_{i+\frac{1}{2}}^{r}, \quad \delta \mathbf{F}_{i+\frac{1}{2}}=\sum(\widetilde{\lambda} \alpha \widetilde{\mathbf{e}})_{i+\frac{1}{2}}^{r} .
$$

The value of the characteristic variables at the left and right side of the intercell position in matrix form, $\mathbf{V}_{i}^{-}$and $\mathbf{V}_{i+1}^{+}$, is:

$\mathbf{V}_{i}^{-}=\mathbf{V}_{i}+\left(\tilde{\boldsymbol{\Lambda}}^{-1} \tilde{\boldsymbol{\Lambda}}^{-} \boldsymbol{\Theta} \delta \mathbf{V}\right)_{i+\frac{1}{2}} \quad \mathbf{V}_{i+1}^{+}=\mathbf{V}_{i+1}-\left(\tilde{\boldsymbol{\Lambda}}^{-1} \tilde{\boldsymbol{\Lambda}}^{+} \boldsymbol{\Theta} \delta \mathbf{V}\right)_{i+\frac{1}{2}}$,

where $\boldsymbol{\Theta}$ is a diagonal matrix

$\boldsymbol{\Theta}_{i+\frac{1}{2}}=\left(\begin{array}{cc}\theta^{1} & 0 \\ 0 & \theta^{2}\end{array}\right)_{i+\frac{1}{2}}, \quad \theta_{i+\frac{1}{2}}^{r}=1-\left(\frac{\beta^{r}}{\lambda^{r} \alpha^{r}}\right)_{i+\frac{1}{2}}$.

Now the intermediate states $\mathbf{U}_{i}^{-}$and $\mathbf{U}_{i+1}^{+}$can be directly obtained by using the $\widetilde{\mathbf{P}}$ matrix. Vector solutions $\mathbf{U}_{i}^{-}=\widetilde{\mathbf{P}} \mathbf{V}_{i}^{-}$and $\mathbf{U}_{i+1}^{+}=\tilde{\mathbf{P}} \mathbf{V}_{i+1}^{+}$are recovered from (59) as follows:

$$
\begin{gathered}
\mathbf{U}_{i}^{-}=\mathbf{U}_{i}+\left(\widetilde{\mathbf{P}} \tilde{\boldsymbol{\Lambda}}^{-1} \tilde{\boldsymbol{\Lambda}}^{-} \Theta \widetilde{\mathbf{P}}^{-1}\right)_{i+\frac{1}{2}} \delta \mathbf{U}_{i+\frac{1}{2}}=\mathbf{U}_{i}+\sum_{\tilde{\lambda}^{r}<0}(\alpha \theta \widetilde{\mathbf{e}})_{i+\frac{1}{2}}^{r}, \\
\mathbf{U}_{i+1}^{+}=\mathbf{U}_{i+1}-\left(\widetilde{\mathbf{P}} \tilde{\boldsymbol{\Lambda}}^{-1} \tilde{\boldsymbol{\Lambda}}^{+} \boldsymbol{\Theta} \widetilde{\mathbf{P}}^{-1}\right)_{i+\frac{1}{2}} \delta \mathbf{U}_{i+\frac{1}{2}}=\mathbf{U}_{i+1}-\sum_{\tilde{\lambda}^{r}>0}(\alpha \theta \widetilde{\mathbf{e}})_{i+\frac{1}{2}}^{r},
\end{gathered}
$$

with the following property:

$$
\mathbf{U}_{i+1}^{+}-\mathbf{U}_{i}^{-}=\left(\widetilde{\mathbf{P}} \tilde{\boldsymbol{\Lambda}}^{-1} \mathbf{B}^{\beta}\right)_{i+\frac{1}{2}}=\widetilde{\mathbf{P}} \widetilde{\boldsymbol{\Lambda}}^{-1} \widetilde{\mathbf{P}}^{-1} \mathbf{S}_{i+\frac{1}{2}}=\widetilde{\mathbf{J}}_{i+\frac{1}{2}}^{-1} \mathbf{S}_{i+\frac{1}{2}}=\mathbf{H}_{i+\frac{1}{2}} .
$$

Being the solution defined as a sum of jumps or shocks between the different intermediate states, the solution for the approximate linear flux function at $x=0, \mathbf{F}_{i}^{-}$and $\mathbf{F}_{i+1}^{+}$, is:

$\mathbf{F}_{i}^{-}=\mathbf{F}_{i}+\sum_{r}\left(\widetilde{\lambda}^{-} \alpha \theta \widetilde{\mathbf{e}}\right)_{i+\frac{1}{2}}^{r}, \quad \mathbf{F}_{i+1}^{+}=\mathbf{F}_{i+1}-\sum_{r}\left(\widetilde{\lambda}^{+} \alpha \theta \widetilde{\mathbf{e}}\right)_{i+\frac{1}{2}}^{r}$. with $\tilde{\lambda}^{ \pm}=\frac{1}{2}(\tilde{\lambda} \pm|\tilde{\lambda}|)$. Also, the relation between the intercell approximate fluxes can be analyzed using the RH (Rankine-Hugoniot) relation at $x=0$ that includes a steady contact wave between approximate solutions $\mathbf{U}_{i}^{-}$and $\mathbf{U}_{i+1}^{+}$

$\mathbf{F}_{i+1}^{+}-\mathbf{F}_{i}^{-}-\overline{\mathbf{S}}_{i+\frac{1}{2}}=\lambda^{0}\left(\mathbf{U}_{i+1}^{+}-\mathbf{U}_{i}^{-}\right)=0$.

It also provides the following relation among fluxes and conserved variables:

$\mathbf{F}_{i+1}^{+}-\mathbf{F}_{i}^{-}=\widetilde{\mathbf{J}}_{i+\frac{1}{2}}\left(\mathbf{U}_{i+1}^{+}-\mathbf{U}_{i}^{-}\right)=\mathbf{S}_{i+\frac{1}{2}}$,

that recovers the relation in (62).

In order to account for the variations in material and geometrical properties of the vessels, the intercell flux in (63) is modified to provide the approximate first order flux in (14), given by:

$$
\begin{aligned}
& \mathbf{F}_{i+\frac{1}{2}}^{\text {ARoo- }}=\left(\mathbf{F}_{i}^{-}-\mathbf{F}_{i}\right)+\mathcal{F}_{i}=\mathcal{F}_{i}+\sum_{r}\left(\widetilde{\lambda}^{-} \alpha \theta \widetilde{\mathbf{e}}\right)_{i+\frac{1}{2}}^{r}, \\
& \mathbf{F}_{i-\frac{1}{2}}^{\text {ARoo }+}=\left(\mathbf{F}_{i}^{+}-\mathbf{F}_{i}\right)+\mathcal{F}_{i}=\mathcal{F}_{i}-\sum_{r}(\widetilde{\lambda}+\alpha \theta \widetilde{\mathbf{e}})_{i-\frac{1}{2}}^{r} .
\end{aligned}
$$

On the other hand, the equivalent fluctuation form of numerical scheme in (13) can be derived by simply using the intercell flux definitions in (66)

$$
\mathbf{U}_{i}^{n+1}=\mathbf{U}_{i}^{n}-\left(\left[\mathcal{F}_{i}+\sum_{r}\left(\widetilde{\lambda}^{-} \alpha \theta \widetilde{\mathbf{e}}\right)_{i+\frac{1}{2}}^{r}\right]-\left[\mathcal{F}_{i}-\sum_{r}\left(\widetilde{\lambda}^{+} \alpha \theta \widetilde{\mathbf{e}}\right)_{i-\frac{1}{2}}^{r}\right]\right) \frac{\Delta t}{\Delta x},
$$

leading to

$$
\delta \mathbf{M}_{i+\frac{1}{2}}^{\text {ARoe- }}=\sum_{r}\left(\widetilde{\lambda}^{-} \alpha \theta \widetilde{\mathbf{e}}\right)_{i+\frac{1}{2}}^{r}, \quad \delta \mathbf{M}_{i-\frac{1}{2}}^{\text {ARoe+ }}=\sum_{r}\left(\widetilde{\lambda}^{+} \alpha \theta \widetilde{\mathbf{e}}\right)_{i-\frac{1}{2}}^{r} .
$$

with

$\delta \mathbf{M}_{i+\frac{1}{2}}^{\text {ARoe- }}+\delta \mathbf{M}_{i+\frac{1}{2}}^{\text {ARoe+ }}=\delta \mathbf{M}_{i+\frac{1}{2}}$.

If functions $\delta \mathbf{M}_{i+\frac{1}{2}}^{ \pm}$satisfy $\delta \mathbf{M}_{i \mp \frac{1}{2}}^{A R o e \pm}=0$ in steady cases, convergence to a solution with mesh refinement is guaranteed.

\subsection{Wave-Speed estimates in the ARoe solver}

Roe's linearization provides a good approximation for contact and shock waves or elastic jumps but, for rarefaction waves, where a continuous change in flow variables appears, the approximations based on shocks may lead to inaccurate results, especially in cases of transonic flow. The accuracy is certainly improved if using the Harten-Hyman entropy fix $[48,49]$ and can be extended to account for the presence of source terms [36]. If a left transonic rarefaction characterized by $\lambda_{i}^{1}<0<\lambda_{i+1}^{1}$ is present in the solution, $\tilde{\lambda}^{1} \rightarrow 0$, to prevent unphysical solutions, the $\tilde{\lambda}^{1}$ celerity and the wave strength $\beta^{1}$ are split in two components, $\widetilde{\lambda}_{a}^{1}, \widetilde{\lambda}_{b}^{1}$ and $\widetilde{\beta}_{a}^{1}$, $\widetilde{\beta}_{b}^{1}$ respectively:

$$
\begin{aligned}
& \tilde{\lambda}_{a, i+\frac{1}{2}}^{1}=\lambda_{i}^{1}\left(\lambda_{i+1}^{1}-\tilde{\lambda}_{i+\frac{1}{2}}^{1}\right)\left(\delta \lambda^{1}\right)_{i+\frac{1}{2}}^{-1}, \\
& \tilde{\lambda}_{b, i+\frac{1}{2}}^{1}=\lambda_{i+1}^{1}\left(\tilde{\lambda}_{i+\frac{1}{2}}^{1}-\lambda_{i}^{1}\right)\left(\delta \lambda^{1}\right)_{i+\frac{1}{2}}^{-1}, \quad \widetilde{\beta}_{a}^{1}=\bar{\beta}^{1}, \quad \widetilde{\beta}_{b}^{1}=0 .
\end{aligned}
$$

In case of a right transonic rarefaction, $\lambda_{i}^{2}<0<\lambda_{i+1}^{2}, \tilde{\lambda}^{2} \rightarrow 0$. The entropy fix procedure is entirely analogous to the left rarefaction case and the $\widetilde{\lambda}^{2}$ celerity and the wave strength $\beta^{2}$ are split again in two components

$$
\begin{aligned}
& \tilde{\lambda}_{a, i+\frac{1}{2}}^{2}=\lambda_{i}^{2}\left(\lambda_{i+1}^{2}-\tilde{\lambda}_{i+\frac{1}{2}}^{2}\right)\left(\delta \lambda^{2}\right)_{i+\frac{1}{2}}^{-1}, \\
& \tilde{\lambda}_{b, i+\frac{1}{2}}^{2}=\lambda_{i+1}^{2}\left(\tilde{\lambda}_{i+\frac{1}{2}}^{2}-\lambda_{i}^{2}\right)\left(\delta \lambda^{2}\right)_{i+\frac{1}{2}}^{-1}, \\
& \widetilde{\beta}_{a, i+\frac{1}{2}}^{2}=0, \quad \widetilde{\beta}_{b, i+\frac{1}{2}}^{2}=\bar{\beta}_{i+\frac{1}{2}}^{2} .
\end{aligned}
$$




\subsection{Positive solution preservation in the ARoe solver}

The numerical integration of the source terms can be performed exactly in steady state RP's but, when moving to transient variations, gross estimations of the source term may lead to unphysical results independently of the time step selected. Inner states in both ARoe and HLLS solvers are determined involving integration of source terms that may lead to negative values of vessel area. In order to avoid unphysical results, the strategy proposed here is based on enforcing positive values of the inner states given by the approximate solution. At each RP, positivity is enforced by ensuring:

$A_{i}^{-}-A_{i}^{\text {coll }} \geq 0, \quad A_{i+1}^{+}-A_{i+1}^{\text {coll }} \geq 0$,

where $A^{\text {coll }}=\alpha^{\text {coll }} A_{o}$, and $\alpha^{\text {coll }}$ is considered the limiting ratio for the collapsed vessels.

It must be noticed that the tube law used for very elastic vessels describes a stiffness that rapidly increases when the vessel is close to achieve the collapse condition. For veins, transmural pressure becomes equal to infinity for $\alpha^{\text {coll }}=0$, so a value of collapsed area equal to zero cannot be handled numerically. This issue is remedied here setting $\alpha^{\text {coll }}=1 . e^{-10}$ in the numerical experiments performed in this work. In this way the numerical scheme will treat solutions with arbitrarily small, but positive, vessel section area, allowing to define time step size based on the celerity of the waves of the system.

It is possible to enforce positivity conditions over the two inner states that shape the approximate solution given by the ARoe solver in the subsonic case, $\widetilde{\lambda}^{1} \widetilde{\lambda}^{2}>0$. The following results can be derived:

- Positive values of $A_{i}^{-}-A_{i}^{\text {coll }} \geq 0$ require the following limit over source term integral $S_{2}$

$S_{2} \leq S_{2, \text { max }}^{\text {Roe }}, \quad S_{2, \text { max }}^{\text {Roe }}=-2\left(A_{i+\frac{1}{2}}^{\star}-A_{i}^{\text {coll }}\right)\left(\widetilde{c}^{1}\right)_{i+\frac{1}{2}}>0$,

with $A_{i+\frac{1}{2}}^{\star}-A_{i}^{\text {coll }}>0$. In case that $A_{i}^{-}-A_{i}^{\text {coll }}$ becomes negative, $S_{2}$ can be replaced by $S_{2, \max }^{R o e}$ with the following consequences:

$$
A_{i}^{-}=A_{i}^{\text {coll }}, \quad A_{i+1}^{+}=A_{i+\frac{1}{2}}^{\star}-\left(A_{i+\frac{1}{2}}^{\star}-A_{i}^{\text {coll }}\right)\left(\frac{\tilde{\lambda}^{1}}{\tilde{\lambda}^{2}}\right)_{i+\frac{1}{2}}>0
$$

- Positive values of $A_{i+1}^{+}>A_{i+1}^{\text {coll }}$, lead to the following limit for $S_{2}$

$S_{2} \geq S_{2, \text { min }}^{\text {Roe }}, \quad S_{2, \text { min }}^{\text {Roe }}=-2\left(A_{i+\frac{1}{2}}^{\star}-A_{i}^{\text {coll }}\right)\left(\widetilde{c} \tilde{\lambda}^{2}\right)_{i+\frac{1}{2}}<0$

provided that $A_{i+\frac{1}{2}}^{\star}-A_{i}^{\text {coll }}>0$. In case that $A_{i+1}^{+}-A_{i+1}^{\text {coll }}$ becomes negative, $S_{2}$ can be replaced by $S_{2 \text {,min }}^{R o e}$ with the following consequences

$A_{i+1}^{+}=A_{i+1}^{\text {coll }}, \quad A_{i}^{-}=A_{i+\frac{1}{2}}^{\star}-\left(A_{i+\frac{1}{2}}^{\star}-A_{i+1}^{\text {coll }}\right)\left(\frac{\widetilde{\lambda}^{2}}{\tilde{\lambda}^{1}}\right)_{i+\frac{1}{2}}>A_{i+1}^{\text {coll }}$

ensuring positive values of water depth in both sides of the solution.

Therefore positives values of $A_{i}^{-}$and $A_{i+1}^{+}$can be ensured if $S_{2, \text { min }}^{\text {Roe }} \leq S_{2} \leq S_{2, \text { max }}^{\text {Roe }}$ when $A_{i+\frac{1}{2}}^{\star}>A_{i+1}^{\text {coll }}$ and $A_{i+\frac{1}{2}}^{\star}>A_{i}^{\text {coll }}$.

\section{The HLLS solver}

The integral average of the approximate RP (40) between the slowest and fastest signals at time $\Delta t$, that will be referred to as
$\overline{\mathbf{U}}$, can be derived by simply setting $x_{L}=\lambda_{L} \Delta t$ and $x_{R}=\lambda_{R} \Delta t$ in the control volume defined in Fig. 1.

$\overline{\mathbf{U}}=\frac{\int_{x_{L}}^{x_{R}} \mathbf{U}(x, \Delta t) d x}{\Delta t\left(\lambda_{R}-\lambda_{L}\right)}=\frac{\lambda_{R} \mathbf{U}_{i+1}-\lambda_{L} \mathbf{U}_{i}-\mathbf{F}_{i+1}+\mathbf{F}_{i}+\mathbf{S}_{i+\frac{1}{2}}}{\left(\lambda_{R}-\lambda_{L}\right)}$

The presence of the source term $\mathbf{S}$ introduces a variation in $\mathbf{U}$ in the solution across $x=0$, leading to two new integral averages $\mathbf{U}_{i}^{-}$and $\mathbf{U}_{i+1}^{+}$

$\mathbf{U}_{i}^{-}=\frac{\int_{x_{L}}^{0} \mathbf{U}(x, \Delta t) d x}{-\Delta t \lambda_{L}}, \quad \mathbf{U}_{i+1}^{+}=\frac{\int_{0}^{x_{R}} \mathbf{U}(x, \Delta t) d x}{\Delta t \lambda_{R}}$.

The introduction of the source term leads also to the definition of fluxes, $\mathbf{F}_{i}^{-}=\mathbf{F}\left(\mathbf{U}_{i}^{-}\right)$and $\mathbf{F}_{i+1}^{+}=\mathbf{F}\left(\mathbf{U}_{i+1}^{+}\right)$, with the following $\mathrm{RH}$ relations across the left and right waves respectively

$\mathbf{F}_{i}^{-}-\mathbf{F}_{i}=\lambda_{L}\left(\mathbf{U}_{i}^{-}-\mathbf{U}_{i}\right), \quad \mathbf{F}_{i+1}-\mathbf{F}_{i+1}^{+}=\lambda_{R}\left(\mathbf{U}_{i+1}-\mathbf{U}_{i+1}^{+}\right)$,

and the RH relation for the steady contact wave, of speed $\lambda_{0}=0$, at $x=0$ in (65). Using this RH relations the intermediate states are given by [37]

$\mathbf{U}_{i+1}^{+}=\mathbf{U}_{i+1 / 2}^{*}+\frac{\mathbf{S}_{i+\frac{1}{2}}-\lambda_{L} \mathbf{H}_{i+\frac{1}{2}}}{\lambda_{R}-\lambda_{L}}, \quad \mathbf{U}_{i}^{-}=\mathbf{U}_{i+1 / 2}^{*}+\frac{\mathbf{S}_{i+\frac{1}{2}}-\lambda_{R} \mathbf{H}_{i+\frac{1}{2}}}{\lambda_{R}-\lambda_{L}}$

The intermediate fluxes are:

$$
\begin{gathered}
\mathbf{F}_{i+1}^{+}=\mathbf{F}_{i+1 / 2}^{*}+\frac{\lambda_{R}\left(\mathbf{S}_{i+\frac{1}{2}}-\lambda_{L} \mathbf{H}_{i+\frac{1}{2}}\right)}{\lambda_{R}-\lambda_{L}}, \\
\mathbf{F}_{i}^{-}=\mathbf{F}_{i+1 / 2}^{*}+\frac{\lambda_{L}\left(\mathbf{S}_{i+\frac{1}{2}}-\lambda_{R} \mathbf{H}_{i+\frac{1}{2}}\right)}{\lambda_{R}-\lambda_{L}},
\end{gathered}
$$

with

$$
\begin{aligned}
\mathbf{U}_{i+1 / 2}^{*} & =\frac{\lambda_{R} \mathbf{U}_{i+1}-\lambda_{L} \mathbf{U}_{i}+\delta \mathbf{F}_{i+\frac{1}{2}}}{\lambda_{R}-\lambda_{L}}, \\
\mathbf{F}_{i+1 / 2}^{*}= & \frac{\lambda_{R} \mathbf{F}_{i}-\lambda_{L} \mathbf{F}_{i+1}+\lambda_{L} \lambda_{R} \delta \mathbf{U}_{i+\frac{1}{2}}}{\lambda_{R}-\lambda_{L}},
\end{aligned}
$$

the solutions for the particular case $\overline{\mathbf{S}}_{i+1 / 2}=\mathbf{0}$.

The corresponding inter cell flux for the approximate Godunov method in (14) is given by two functions adapted here to include the variation in geometrical and mechanical properties of the vessel

$\mathbf{F}_{i+1 / 2}^{H L L S-}=\left\{\begin{array}{ccc}\mathcal{F}_{i} & \text { if } & 0 \leq \lambda_{L} \\ \left(\mathbf{F}_{i+1 / 2}^{*}-\mathbf{F}_{i}\right)+\frac{\lambda_{L}\left(\mathbf{S}_{i+1 / 2}-\lambda_{R} \mathbf{H}_{i+1 / 2}\right)}{\lambda_{R}-\lambda_{L}}+\mathcal{F}_{i} & \text { if } & \lambda_{L} \leq 0 \leq \lambda_{R}, \\ \delta \mathbf{F}_{i+\frac{1}{2}}-\mathbf{S}_{i+1 / 2}+\mathcal{F}_{i} & \text { if } & 0 \geq \lambda_{R}\end{array}\right.$

$\mathbf{F}_{i+1 / 2}^{H L L S+}=\left\{\begin{array}{ccc}-\left(\delta \mathbf{F}_{i+\frac{1}{2}}-\mathbf{S}_{i+1 / 2}+\mathcal{F}_{i+1}\right) & \text { if } & 0 \leq \lambda_{L} \\ \left(\mathbf{F}_{i+1 / 2}^{*}-\mathbf{F}_{i+1}\right)+\frac{\lambda_{R}\left(\mathbf{S}_{i+1 / 2}-\lambda_{L} \mathbf{H}_{i+1 / 2}\right)}{\lambda_{R}-\lambda_{L}}+\mathcal{F}_{i+1} & \text { if } & \lambda_{L} \leq 0 \leq \lambda_{R} . \\ \mathcal{F}_{i+1} & \text { if } & 0 \geq \lambda_{R}\end{array}\right.$

If expressed in fluctuation form, the approximate Godunov method results in:

$\delta \mathbf{M}^{H L L S-}=\left\{\begin{array}{ccc}0 & \text { if } & 0 \leq \lambda_{L} \\ \left(\mathbf{F}_{i+1 / 2}^{*}-\mathbf{F}_{i}\right)+\frac{\lambda_{L}\left(\mathbf{S}_{i+1 / 2}-\lambda_{R} \mathbf{H}_{i+1 / 2}\right)}{\lambda_{R}-\lambda_{L}} & \text { if } & \lambda_{L} \leq 0 \leq \lambda_{R}, \\ \delta \mathbf{F}_{i+\frac{1}{2}}-\mathbf{S}_{i+1 / 2} & \text { if } & 0 \geq \lambda_{R}\end{array}\right.$ 


$$
\delta \mathbf{M}^{H L L S+}=\left\{\begin{array}{ccc}
-\left(\delta \mathbf{F}_{i+\frac{1}{2}}-\mathbf{S}_{i+1 / 2}\right) & \text { if } & 0 \leq \lambda_{L} \\
\left(\mathbf{F}_{i+1 / 2}^{*}-\mathbf{F}_{i+1}\right)+\frac{\lambda_{R}\left(\mathbf{S}_{i+1 / 2}-\lambda_{L} \mathbf{H}_{i+1 / 2}\right)}{\lambda_{R}-\lambda_{L}} & \text { if } & \lambda_{L} \leq 0 \leq \lambda_{R} . \\
0 & \text { if } & 0 \geq \lambda_{R}
\end{array}\right.
$$

In order to generate the intercell fluxes it is necessary to compute the speeds $\lambda_{R}$ and $\lambda_{L}$. Suitable approximations will be discussed next.

\subsection{Wave-Speed estimates in the HLLS solver}

Appropriate estimates for the wave speeds $\lambda_{L}$ and $\lambda_{R}$ are required when computing the numerical fluxes in the HLLS solver. Direct wave speed estimates, $\lambda_{L}=(u-c)_{L}$ and $\lambda_{R}=(u-c)_{R}$, are the simplest methods providing minimum and maximum signal velocities [50] and can be combined with the Roe averages [51]. Also, estimates $\lambda_{L}^{\min }$ and $\lambda_{R}^{\max }$ can also be defined [48]

$$
\begin{aligned}
& \lambda_{L}^{\min }=\min \left(u_{i}, u_{i+1}\right)-\max \left(c_{i}, c_{i+1}\right) \\
& \lambda_{R}^{\max }=\max \left(u_{i}, u_{i+1}\right)+\max \left(c_{i}, c_{i+1}\right) .
\end{aligned}
$$

It is worth noting that even if always selecting the direct estimations to estimate $\lambda_{L}$ and $\lambda_{R}$, the Roe average celerities $\tilde{\lambda}^{m}$ actively participate in the approximate solution through the vector

$\mathbf{H}=\mathbf{U}_{i+1}^{+}-\mathbf{U}_{i}^{-}=\left(\begin{array}{c}H_{1} \\ H_{2}\end{array}\right)=\left(\begin{array}{c}H_{1} \\ 0\end{array}\right), \quad H_{1}=-\frac{S_{2}}{\tilde{\lambda}^{1} \tilde{\lambda}^{2}}$,

where the jump in vessel area produced by the presence of the source terms is defined by $H_{1}$. In $H_{1}$ both wave celerities $\tilde{\lambda}^{1}$ and $\tilde{\lambda}^{2}$ appear, and may produce numerical oscillations or unphysical values in cases of transonic flow, as happens for the Roe solver. Under this circumstance the computation of the jump across the inner solutions in (88) may become unstable, as $H_{1}$ can become excessively large. It is possible to remedy this situation by using an entropy-source fix where wave speed estimates are selected as follows:

- In left transcritical problems when $\lambda_{i}^{1}<0<\lambda_{i+1}^{1}$ set:

$$
\lambda_{L}=\min \left(\lambda_{i}^{1}, \lambda_{L}^{\min }\right), \quad \lambda_{R}=\max \left(\tilde{\lambda}^{2}, \lambda_{i+1}^{2}\right), \quad \tilde{\lambda}^{1}=\lambda_{L}
$$

- In right transcritical problems when $\lambda_{i}^{2}<0<\lambda_{i+1}^{2}$ set:

$$
\lambda_{L}=\min \left(\tilde{\lambda}^{1}, \lambda_{i}^{1}\right), \quad \lambda_{R}=\max \left(\lambda_{R}^{\max }, \lambda_{i+1}^{2}\right), \quad \tilde{\lambda}^{2}=\lambda_{R}
$$

- Otherwise wave celerities are selected setting

$$
\lambda_{L}=\min \left(\tilde{\lambda}^{1}, \lambda_{i}^{1}\right), \quad \lambda_{R}=\max \left(\tilde{\lambda}^{2}, \lambda_{i+1}^{2}\right)
$$

With this selection (88) become stable in transonic problems.

\subsection{Positive solution preservation for the HLLS}

Again, it is possible to enforce positivity conditions over the two inner states that shape the approximate solution given by the HLLS solver. In the subsonic case, $\lambda_{L} \lambda_{R}<0$ the following results can be derived:

- Positive values of $A_{i}^{-}-A_{i}^{\text {coll }} \geq 0$, require the following limit over the source term integral $S_{2}$

$S_{2} \leq S_{2, \max }^{H L L}, \quad S_{2, \max }^{H L L}=-\left(A_{i+\frac{1}{2}}^{\star}-A_{i}^{\text {coll }}\right)\left(\widetilde{\lambda}^{1} \tilde{\lambda}^{2}\right)_{i+\frac{1}{2}}\left(1-\frac{\lambda_{L}}{\lambda_{R}}\right)>0$,

with $A_{i+\frac{1}{2}}^{\star}>A_{i}^{\text {coll }}$, being $A_{i+\frac{1}{2}}^{\star}$ the solution for the homogeneous case without source terms. In case that $A_{i}^{-}$becomes negative,
$S_{2}$ can be replaced by $S_{2, \max }^{H L L}$, leading to the following left and right inner states

$$
A_{i}^{-}=A_{i}^{\text {coll }}, \quad A_{i+1}^{+}=A_{i+\frac{1}{2}}^{\star}+\left(A_{i+\frac{1}{2}}^{\star}-A_{i}^{\text {coll }}\right)\left(-\frac{\lambda_{L}}{\lambda_{R}}\right)_{i+\frac{1}{2}}>0,
$$

ensuring positive values of vessel area on the right side of the plane solution.

- Positive values of $A_{i+1}^{+}-A_{i+1}^{\text {coll }} \geq 0$ lead to the following limit for $\mathrm{S}_{2}$

$S_{2} \geq S_{2, \text { min }}^{H L L}, \quad S_{2, \text { min }}^{H L L}=\left(A_{i+\frac{1}{2}}^{\star}-A_{i+1}^{\text {coll }}\right)\left(\tilde{\lambda}^{1} \tilde{\lambda}^{2}\right)_{i+\frac{1}{2}}\left(1-\frac{\lambda_{R}}{\lambda_{L}}\right)<0$,

provided that $A_{i+\frac{1}{2}}^{\star}>A_{i+1}^{\text {coll }}$. In case that $A_{i+1}^{+}$becomes negative, $S_{2}$ can be replaced by $S_{2 \text {,min }}^{H L L}$ with the following consequences

$$
A_{i+1}^{+}=A_{i+1}^{\text {coll }}, \quad A_{i}^{-}=A_{i+\frac{1}{2}}^{\star}+\left(A_{i+\frac{1}{2}}^{\star}-A_{i+1}^{\text {coll }}\right)\left(-\frac{\lambda_{R}}{\lambda_{L}}\right)_{i+\frac{1}{2}}>0,
$$

ensuring positive positive values of vessel area in both sides of the solution.

Therefore positive values of $A_{i}^{-}$and $A_{i+1}^{+}$can be ensured if $S_{2, \text { min }}^{H L L} \leq S_{2} \leq S_{2, \text { max }}^{H L L}$ when $A_{i+\frac{1}{2}}^{\star}>A_{i+1}^{\text {coll }}$ and $A_{i+\frac{1}{2}}^{\star}>A_{i}^{\text {coll }}$.

\section{Summary of the ARoe and HLLS solvers}

In this section, the necessary steps required to compute the intercell fluxes in (66) or fluctuation waves in (68) at each $i+\frac{1}{2}$ cell edge are listed. In this procedure all quantities are calculated explicitly. For the ARoe the procedure is defined as follows:

1. Compute the fluxes $\mathcal{F}_{i}=\mathcal{F}\left(\mathbf{U}_{i}\right)$, with $\mathcal{F}$ defined as in (2).

2. Compute the dimensionless variable $\Pi_{E}$ using (47) and the approximate vessel area $\mathcal{A}$ using (48).

3. Compute the pulse wave velocity average value $\tilde{c}$ and the source term $S_{2}$ depending on the flow conditions defined in 49-(51).

4. Compute eigenvalues $\tilde{\lambda}^{1,2}$ and eigenvectors $\widetilde{\mathbf{e}}^{1,2}$.

5. Limit the value of the source term $S_{2}, S_{2, \min }^{\text {Roe }} \leq S_{2} \leq S_{2, \text { max }}^{\text {Roe }}$, to ensure positive values of vessel area.

6. Compute wave strengths $\alpha^{1,2}$, source strengths $\bar{\beta}^{1,2}$ and coefficients $\theta^{1,2}$ in (57), (55) and (60) respectively.

7. Split the eigenvalues $\lambda^{1,2}$ using the Harten-Hyman entropy fix as defined in (70) and (71) in cases of transonic flow.

8. Use summations in (66) or (68) to define the update the solution using numerical fluxes in (14) or using fluctuations in (15) respectively.

On the other hand, for the HLLS the procedure is defined as follows:

1. Compute the fluxes $\mathcal{F}_{i}=\mathcal{F}\left(\mathbf{U}_{i}\right)$, with $\mathcal{F}$ defined as in (2).

2. Compute the dimensionless variable $\Pi_{E}$ using (47) and the approximate vessel area $\mathcal{A}$ using (48).

3. Compute the pulse wave velocity average value $\tilde{c}$ and the source term $S_{2}$ depending on the flow conditions defined in 49-(51).

4. Compute new flux functions $\mathbf{F}_{i}$ and $\mathbf{F}_{i+1}$ using definition in (38).

5. Compute wave speeds $\tilde{\lambda}^{1,2}, \lambda_{L}$ and $\lambda_{R}$ depending on the flow conditions in 89-(91).

6. Limit the value of the source term $S_{2}, S_{2, \min }^{H L L S} \leq S_{2} \leq S_{2 \text {,max }}^{H L L S}$, to ensure positive values of vessel area. 


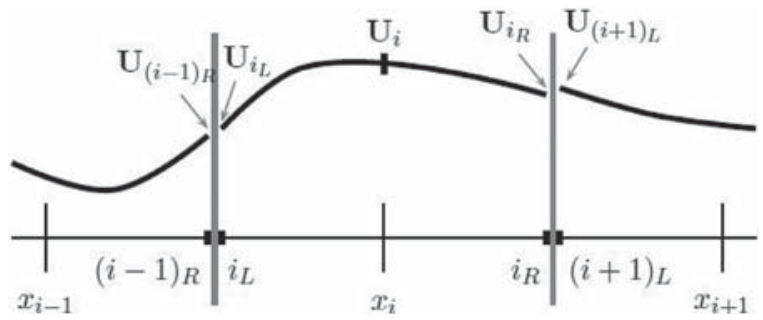

Fig. 2. Mesh discretization.

7. Compute $\mathbf{U}_{i+1 / 2}^{*}$ and $\mathbf{F}_{i+1 / 2}^{*}$ in (82) and vector $\mathbf{H}_{i+1 / 2}$ in (88).

8. Use summations in (83) or (85) to define the updated solution using numerical fluxes in (14) or using fluctuations in (15) respectively.

As in both solvers the integration of source term is numerically controlled, only wave celerities define the maximum allowable time step. Considering that the grid size $\Delta x$ is fixed, the time step $\Delta t$ is computed considering the speed of the fastest wave, leading to the following limit:

$$
\Delta t \leq C F L \frac{\Delta x}{\max \left(c_{i}+|u|_{i}\right)}, \quad C F L \leq 1,
$$

where a CFL value equal to $1 / 2$ ensures no interaction of waves from neighboring Riemann problems.

\section{Extension to high order}

Updating formulae in (14) or (13) can be expressed in the following form

$\mathbf{U}_{i}^{n+1}=\mathbf{U}_{i}^{n}+\Delta t \hbar\left(\mathbf{U}^{n}, t^{n}\right)$,

with $\hbar$ an operator of the set of approximate solutions at time $t^{n}$, $\mathbf{U}^{n}$,

$\hbar\left(\mathbf{U}^{n}, t^{n}\right)=-\frac{1}{\Delta x}\left[\mathbf{F}_{i+\frac{1}{2}}^{-}-\mathbf{F}_{i-\frac{1}{2}}^{+}\right]^{n}=-\frac{1}{\Delta x}\left[\delta \mathbf{M}_{i+\frac{1}{2}}^{-}+\delta \mathbf{M}_{i-1 / 2}^{+}\right]^{n}$

Following the approach proposed in $[22,39]$ a suitable arbitraryorder extension of the previous method can be constructed redefining the $\hbar$ operator as

$$
\begin{aligned}
\hbar(\mathbf{U}) & =-\frac{1}{\Delta x}\left[\mathbf{F}_{i+1 / 2}^{-}-\mathbf{F}_{i-1 / 2}^{+}\right]+\frac{1}{\Delta x}\left[\mathbf{S}_{i_{R}, i_{L}}\right] \\
& =-\frac{1}{\Delta x}\left[\delta \mathbf{M}_{i+1 / 2}^{-}+\delta \mathbf{M}_{i_{R}, i_{L}}+\delta \mathbf{M}_{i-1 / 2}^{+}\right],
\end{aligned}
$$

where now numerical fluxes and wave fluctuations used to evaluate $\hbar(\mathbf{U})$ will be computed using the WENO reconstruction as initial data for the RPs,

$\mathbf{F}_{i+\frac{1}{2}}=\mathbf{F}_{i+\frac{1}{2}}\left(\mathbf{U}_{i_{R}}, \mathbf{U}_{(i+1)_{L}}\right), \quad \delta \mathbf{M}_{i+\frac{1}{2}}=\delta \mathbf{M}_{i+\frac{1}{2}}\left(\mathbf{U}_{i_{R}}, \mathbf{U}_{(i+1)_{L}}\right)$,

with $\mathbf{U}_{i_{R}}$ and $\mathbf{U}_{(i+1)_{L}}$ the reconstructed value of the right and left states of $\mathbf{U}$ at the cells $i$ and $i+1$ respectively, as depicted in Fig. 2. Note that in (99) new extra terms that account for centered contributions appear. Such terms are a suitable approximation of the integral of the source term inside the cell, $\mathbf{S}_{i_{R}, i_{L}}$, and a centered wave fluctuation, $\delta \mathbf{M}_{i_{R}, i_{L}}$, given by $[22,39]$

$\mathbf{S}_{i_{R}, i_{L}}=\mathbf{S}_{i_{R}, i}+\mathbf{S}_{i, i_{L}}, \quad \delta \mathbf{M}_{i_{R}, i_{L}}=\mathbf{F}_{i_{R}}-\mathbf{F}_{i_{L}}-\mathbf{S}_{i_{R}, i_{L}}$.

The approach in (99) allows to construct a numerical scheme of high order of accuracy in space. In order to provide a high order accuracy also in time, an optimal third-order TVDRK3 method [54] is used. In contrast to the widespread one-step ADER approach, the TVDRK3 requires 3 integration steps but avoids the use of the Cauchy-Kovalewski procedure, which may become inaccurate in presence of stiff source terms [56]. The TVDRK3 is constructed as follows [57] :

$\mathbf{U}_{i}^{(1)}=\mathbf{U}_{i}^{n}+\Delta t \hbar\left(\mathbf{U}^{n}\right)$,

$\mathbf{U}_{i}^{(2)}=\frac{3}{4} \mathbf{U}_{i}^{n}+\frac{1}{4} \mathbf{U}_{i}^{(1)}+\frac{1}{4} \Delta t \hbar\left(\mathbf{U}^{(1)}\right)$,

$\mathbf{U}_{i}^{n+1}=\frac{1}{3} \mathbf{U}_{i}^{n}+\frac{2}{3} \mathbf{U}_{i}^{(2)}+\frac{2}{3} \Delta t \hbar\left(\mathbf{U}^{(2)}\right)$,

where the operator $\hbar(\mathbf{U}, t)$ is evaluated using the WENO reconstructions after each updating stage.

In order to preserve equilibrium in exactly balanced cases involving movement, WENO reconstructions are performed over the flow $Q$ over the prescribed external pressure and gravity effects combined in $p_{d}=p_{e}+g \rho \eta$, over the vessel stiffness $K$ and over the geometrical properties $A_{0}$ and $p_{0}$. Vessel area is not reconstructed directly by means of the WENO method. Instead, total energy $P_{t}=p+\rho \frac{u^{2}}{2}+p_{d}$ is reconstructed departing from $P_{t}$ cell averaged values in each computational cell. The reconstructed value of $P_{t}$ can be next expressed in terms of the previously reconstructed variables, and the single remaining unknown variable is the vessel area $A$. For more details we refer the reader to the work in [60], where the validity of this method is proved.

The third-order WENO reconstruction over an arbitrary $q$ function can be written in the following form [55]

$q_{i_{R}}=w_{0}\left(-\frac{1}{2} q_{i-1}+\frac{3}{2} q_{i}\right)+w_{1}\left(\frac{1}{2} q_{i}+\frac{1}{2} q_{i+1}\right)$,

$q_{i_{L}}=w_{0}\left(\frac{1}{2} q_{i}+\frac{1}{2} q_{i-1}\right)+w_{1}\left(-\frac{1}{2} q_{i+1}+\frac{3}{2} q_{i}\right)$,

where nonlinear weights $w_{k}$ are defined as

$w_{k}=\alpha_{k} /\left(\alpha_{0}+\alpha_{1}\right), \quad \alpha_{k}=d_{k} /\left(\beta_{k}+\epsilon\right)^{2}, \quad k=0,1$

with the smoothness indicators $\beta_{k}$ given by $\beta_{0}=\left(q_{i}-q_{i-1}\right)^{2}$ and $\beta_{1}=\left(q_{i+1}-q_{i}\right)^{2}$. The optimal linear weighting coefficients are $d_{0}=1 / 3$ and $d_{1}=2 / 3$ in $q_{i+1 / 2}^{L}$, and $d_{0}=1 / 3$ and $d_{1}=2 / 3$ in $q_{i-1 / 2}^{R}$, with $\epsilon=10^{-6}$ for eliminating zero denominators.

\section{Results}

\subsection{Empirical convergence rate}

In order to verify that the expected order of accuracy is reached when using the third order WENO reconstruction in combination with the TVDRK3 method, we perform a numerical convergence rate study. The following initial conditions and parameters are imposed in the vessel:

$A(x, t=0)=4.0+0.04 \sin (2.0 \pi x / L)$,

$A_{o}(x, t=0)=4.0+0.04 \sin (2.0 \pi x / L)$,

$K(x, t=0)=50000.0+5.0 \sin (2.0 \pi x / L)$,

$p_{e}(x, t=0)=5.0 \sin (2.0 \pi x / L)$,

with $A$ and $A_{o}(x)$ in $\mathrm{cm}^{2}, K$ and $p_{e}$ in Pa and $L=1.0 \mathrm{~m}$. The vessel is connected at both ends, thus forming a closed loop with cyclic boundary conditions. Numerical solutions are computed setting $C F L=0.45$ and compared at $t=1 \mathrm{~s}$. The order of the truncation error of the solutions can be estimated from the results for different mesh sizes using the double-mesh principle [58,59], provided that they have a set of mesh points in common. Here mesh refinement is done with a constant cell ratio $r_{\Delta x}=2$. Numerical results for vessel area factor $\alpha$ are shown in Table 1, where $N$ is the number of cells. Both solvers provide identical values in this case. This result was pointed out in [22] (tables 9 and 10). The expected convergence rate is reached for norms $L_{1}$ and $L_{2}$ for both solvers. 
Table 1

Convergence results for the HLLS and ARoe schemes using the third order WENO reconstruction in combination with the TVDRK3 method.

\begin{tabular}{lllllllll}
\hline $\mathrm{N}$ & $L_{1}^{\text {HLLS }}$ & $L_{2}^{\text {HLLS }}$ & $\mathcal{O}\left(L_{1}\right)^{\text {HLLS }}$ & $\mathcal{O}\left(L_{2}\right)^{\text {HLLS }}$ & $L_{1}^{\text {ARoe }}$ & $L_{2}^{\text {ARoe }}$ & $\mathcal{O}\left(L_{1}\right)^{\text {ARoe }}$ & $\mathcal{O}\left(L_{2}\right)^{\text {ARoe }}$ \\
\hline 50 & $2.18 \mathrm{e}-03$ & $2.50 \mathrm{e}-04$ & & & $2.18 \mathrm{e}-03$ & $2.50 \mathrm{e}-04$ & & \\
100 & $3.08 \mathrm{e}-04$ & $3.65 \mathrm{e}-05$ & 2.82 & 2.78 & $3.08 \mathrm{e}-04$ & $3.65 \mathrm{e}-05$ & 2.82 & 2.78 \\
200 & $4.26 \mathrm{e}-05$ & $5.54 \mathrm{e}-06$ & 2.85 & 2.72 & $4.26 \mathrm{e}-05$ & $5.54 \mathrm{e}-06$ & 2.85 & 2.72 \\
400 & $5.53 \mathrm{e}-06$ & $7.26 \mathrm{e}-07$ & 2.95 & 2.93 & $5.53 \mathrm{e}-06$ & $7.26 \mathrm{e}-07$ & 2.95 & 2.93 \\
800 & $7.05 \mathrm{e}-07$ & $9.34 \mathrm{e}-08$ & 2.97 & 2.96 & $7.05 \mathrm{e}-07$ & $9.34 \mathrm{e}-08$ & 2.97 & 2.96 \\
1600 & $9.31 \mathrm{e}-08$ & $1.23 \mathrm{e}-08$ & 2.92 & 2.93 & $9.31 \mathrm{e}-08$ & $1.23 \mathrm{e}-08$ & 2.92 & 2.93 \\
3200 & $1.17 \mathrm{e}-08$ & $1.36 \mathrm{e}-09$ & 2.99 & 3.17 & $1.17 \mathrm{e}-08$ & $1.36 \mathrm{e}-09$ & 2.99 & 3.17 \\
\hline
\end{tabular}

Table 2

Left and right mechanical and geometrical properties and initial conditions.

\begin{tabular}{lllllll}
\hline$p_{e, L}(\mathrm{mmHg})$ & $A_{L, o}\left(\mathrm{~cm}^{2}\right)$ & $K_{L}(\mathrm{~Pa})$ & $A_{L}\left(\mathrm{~cm}^{2}\right)$ & $u_{L}(\mathrm{~m} / \mathrm{s})$ & $m$ & $n$ \\
0.0 & 5.0 & 17888.54382 & 12 & 2 & 0.50 & 0 \\
$p_{e, R}(\mathrm{mmHg})$ & $A_{R, o}\left(\mathrm{~cm}^{2}\right)$ & $K_{R}(\mathrm{~Pa})$ & $A_{R}\left(\mathrm{~cm}^{2}\right)$ & $u_{R}(\mathrm{~m} / \mathrm{s})$ & $m$ & $n$ \\
5.0 & 4.0 & 24000 & 7.63647408600032 & 3.14281168635121 & 0.50 & 0 \\
\hline
\end{tabular}
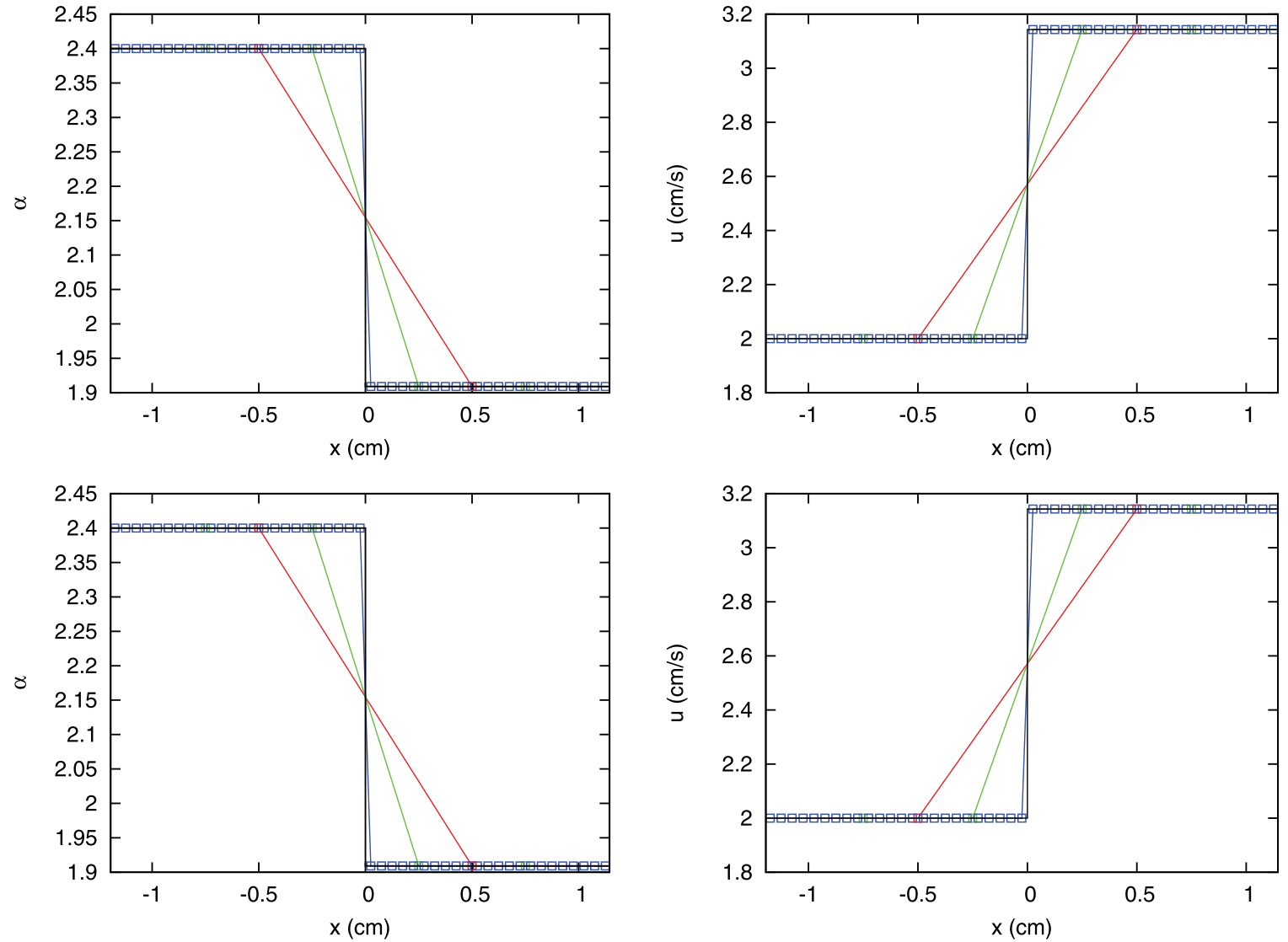

Fig. 3. Section 8.2. Exactly balanced RP. Exactly balanced Riemann problem. Comparison between exact (--) and numerical solutions at $t=0.005 \mathrm{~s}$ using the first order (upper) and TVDRK3 method (lower) using $\Delta x=1$ (red), 0.5 (green), 0.05 (blue) cm, for the HLLS solver $-\circ-$ and ARoe solver $-\square-$. (For interpretation of the references to colour in this figure legend, the reader is referred to the web version of this article.)

\subsection{An exactly balanced riemann problem}

This RP is designed to test the well-balanced properties of both the ARoe and the HLLS schemes. Initial conditions are defined in Table 2. The problem presents variations in vessel wall stiffness $K$, cross-sectional area at reference state $A_{0}$ and external pressure $p_{e}$. In this case flow, momentum and energy are exactly balanced, therefore the exact solution of this problem is identically the initial condition.

Numerical solutions for factor $\alpha$ and velocity $u$ using the HLLS and ARoe solvers are plotted in Fig. 3 at $t=0.005 \mathrm{~s}$ for the three levels of mesh refinement using the first order and the 3th or- der WENO in combination with the TVDRK3 method. Both solvers preserve the initial condition with machine precission (i.e. $L_{\infty} \approx$ $10^{-14}$ ) for the three levels of mesh refinement.

When analyzing the SWE, hydrostatic or gravity forces and friction forces have the same effect on the flow than the observed in the governing equations for blood flow in elastic vessels, and they can be formulated exactly in the same way. Then, if variations in external pressure can be defined as source terms that give rise to well-balancing problems, friction and gravity can be treated in the same fashion, and not as algebraic source terms which can cause problems because of stiffness, not well-balancing. 
Table 3

Left and right mechanical and geometrical properties and initial conditions.

\begin{tabular}{lllllll}
\hline Test & $A_{L, o}\left(\mathrm{~cm}^{2}\right)$ & $K_{L}(\mathrm{~Pa})$ & $A_{L}\left(\mathrm{~cm}^{2}\right)$ & $u_{L}(\mathrm{~m} / \mathrm{s})$ & $m$ & $n$ \\
\hline 1 & 5 & 17888.54382 & 12 & 2 & 0.50 & 0 \\
2 & 5 & 17888.54382 & 8 & 0 & 0.50 & 0 \\
3 & 5 & 17888.54382 & 8 & 40 & 0.50 & 0 \\
4 & 1 & 0.919219 & 0.01 & 2.353043016 & 0.50 & 0 \\
5 & 1 & 0.919219 & 0.01 & 2.353043016 & 0.50 & 0 \\
6 & 0.282743339 & 2000 & 0.32 & 10 & 10.00 & -1.5 \\
7 & 0.282743339 & 33.33333333 & 0.29 & 20 & 10.00 & -1.5 \\
8 & 0.282743339 & 33.33333333 & 0.342 & 50 & 10.00 & -1.5 \\
9 & 0.282743339 & 33.33333333 & 0.31 & -20 & 10.00 & -1.5 \\
Test & $A_{R, o}\left(\mathrm{~cm}^{2}\right)$ & $K_{R}(\mathrm{~Pa})$ & $A_{R}\left(\mathrm{~cm}^{2}\right)$ & $u_{R}(\mathrm{~m} / \mathrm{s})$ & $m$ & $n$ \\
1 & 4 & 24000 & 4.503506204 & 0 & 0.50 & 0 \\
2 & 4 & 24000 & 6 & 0 & 0.50 & 0 \\
3 & 4 & 24000 & 6.015212661 & 0 & 0.50 & 0 \\
4 & 1 & 0.781336 & 0.01243004 & 2.25423876 & 0.50 & 0 \\
5 & 1 & 0.781336 & 0.1 & 2.25423876 & 0.50 & 0 \\
6 & 0.311017673 & 33.33333333 & 0.32 & 20 & 10.00 & -1.5 \\
7 & 0.296880506 & 3333.333333 & 0.32 & 10 & 10.00 & -1.5 \\
8 & 0.32515484 & 1333.333333 & 0.334 & -10 & 10.00 & -1.5 \\
9 & 0.296880506 & 1000 & 0.31 & 10 & 10.00 & -1.5 \\
\hline
\end{tabular}

When considering gravity forces, hydrostatic pressure variations arise from changes in vessel elevation $\eta$, and their effect is equivalent to the application of external pressure variations along the length of the vessel. Both source terms appear in the momentum equation and can be transformed into an equivalent pressure

$\frac{A}{\rho} \frac{\partial p_{e}}{\partial x}+g A \frac{\partial \eta}{\partial x}=\frac{A}{\rho} \frac{\partial}{\partial x}\left(p_{e}+\rho g \eta\right)=\frac{A}{\rho} \frac{\partial p_{e}^{\star}}{\partial x}$

with $p_{e}^{\star}(x)=p_{e}(x)+\rho g \eta(x)$. Therefore, variations in vessel external pressure can be exactly transformed in variations in vessel elevation using

$\delta \eta=-\frac{\delta p_{e}}{\rho g}$.

This transformation allows to express pressure using dimensions of length. Then, as the gravity term has been included in the variations of the driving pressure $\delta p_{d, i+1 / 2}$, the resulting numerical scheme ensures the preservation of the initial condition in exactly balanced problems not only in presence of variations of the external pressure, but also in cases involving variations in vessel elevation. In this way, when the jump in external pressure $\left(p_{e, R}-p_{e, L}=5 \mathrm{mmHg}\right)$ imposed at the discontinuity of the RP presented in this section is replaced by a jump in the vessel elevation $\left(\eta_{e, R}-\eta_{e, L}=-6.45 \mathrm{~cm}\right)$, the numerical solution reproduces exactly to the same results, ensuring again the exactly balanced property.

\subsection{Riemann problems with exact solutions.}

In this section exact solutions are compared with numerical solutions provided by the numerical schemes in a broad variety of cases. All numerical test cases are computed setting $C F L=0.45$ and using three levels of mesh refinement, from a very coarse mesh with $\Delta x=1$, to $\Delta x=0.5$ and $\Delta x=0.05 \mathrm{~cm}$. The minimum value of the area for a vessel is computed using the limiting ratio for the collapsed vessels $\alpha^{\text {coll }}=\frac{A^{\text {coll }}}{A_{o}}$, set here as $1 . e^{-10}$ for all vessels.

The exact solutions have been derived using the work presented by Toro and Siviglia in [17]. Their solution algorithm is well-suited for assessing the performance of numerical methods intended for simulating general situations.

Different RP's are listed in Table 3. Test cases 1 to 5, represent different combinations of wave patterns between two arteries with variable geometrical and mechanical properties in subsonic and supersonic Riemann problems. Test cases 6 to 9 will be used to compare exact solutions with numerical predictions for veins with variable geometrical mechanical properties. Test cases 1,2,3 and 4 were proposed in [38] and test cases 6,7, 8 and 9 were proposed in [17].

The solutions of subsonic test case 1 , for dimensionless area factor $\alpha$, velocity and total energy $\frac{u^{2}}{2}+\frac{p_{d}}{\rho}$, are presented in Fig. 4 (upper). The solution includes a left moving rarefaction wave, a steady contact wave at the vessel junction and a strong elastic jump that develops and moves upstream. The numerical solutions obtained with the first order HLLS and ARoe solvers are plotted at $t=0.05 \mathrm{~s}$ for the three levels of mesh refinement. Fan expansion and shock position are accurately captured and numerical diffusion decreases with grid refinement. The numerical solution converges to the exact solution, preserving a correct value of total energy at the initial discontinuity. Numerical results for the ARoe and HLLS solvers are indistinguishable. When the 3th order WENO in combination with the TVDRK3 method is applied the solution is improved providing the same level of accuracy for both solvers.

The exact solution for subsonic test case 2 is also a combination of a rarefaction and an elastic jump. Now, the flow moves backwards generating a right moving rarefaction wave, a steady contact wave at the vessel junction and a left moving elastic jump. Numerical solutions in Fig. 5 show how the ARoe and the HLLS schemes provide accurate and indistinguishable results for both first and third order for the different levels of mesh refinement.

Fig. 6displays the solution for subsonic test case 3 and contains a left-moving elastic jump, a steady contact wave at the discontinuity and a right-moving elastic jump wave. Again numerical diffusion decreases with grid refinement and extension from one to third order. Numerical results for the ARoe and the HLLS solvers are indistinguishable and both methods provide very good agreement with the exact solution and show no oscillation near the discontinuity regions.

Supersonic test case 4 in Fig. 7 includes a stationary contact discontinuity followed by two rarefactions in opposite directions. Both the first order and third order numerical solutions correctly capture the propagation velocity of the rarefaction and transition between both sides of the stationary contact discontinuity. Third order numerical results for $\alpha$ and $u$ show a mild overshoot of area at the onset of the left rarefaction wave, $x \approx 14$, balanced by a mild undershoot at the middle of the left rarefaction wave, $x \approx 12$. The same behavior is noticed when observing the right rarefaction wave. 

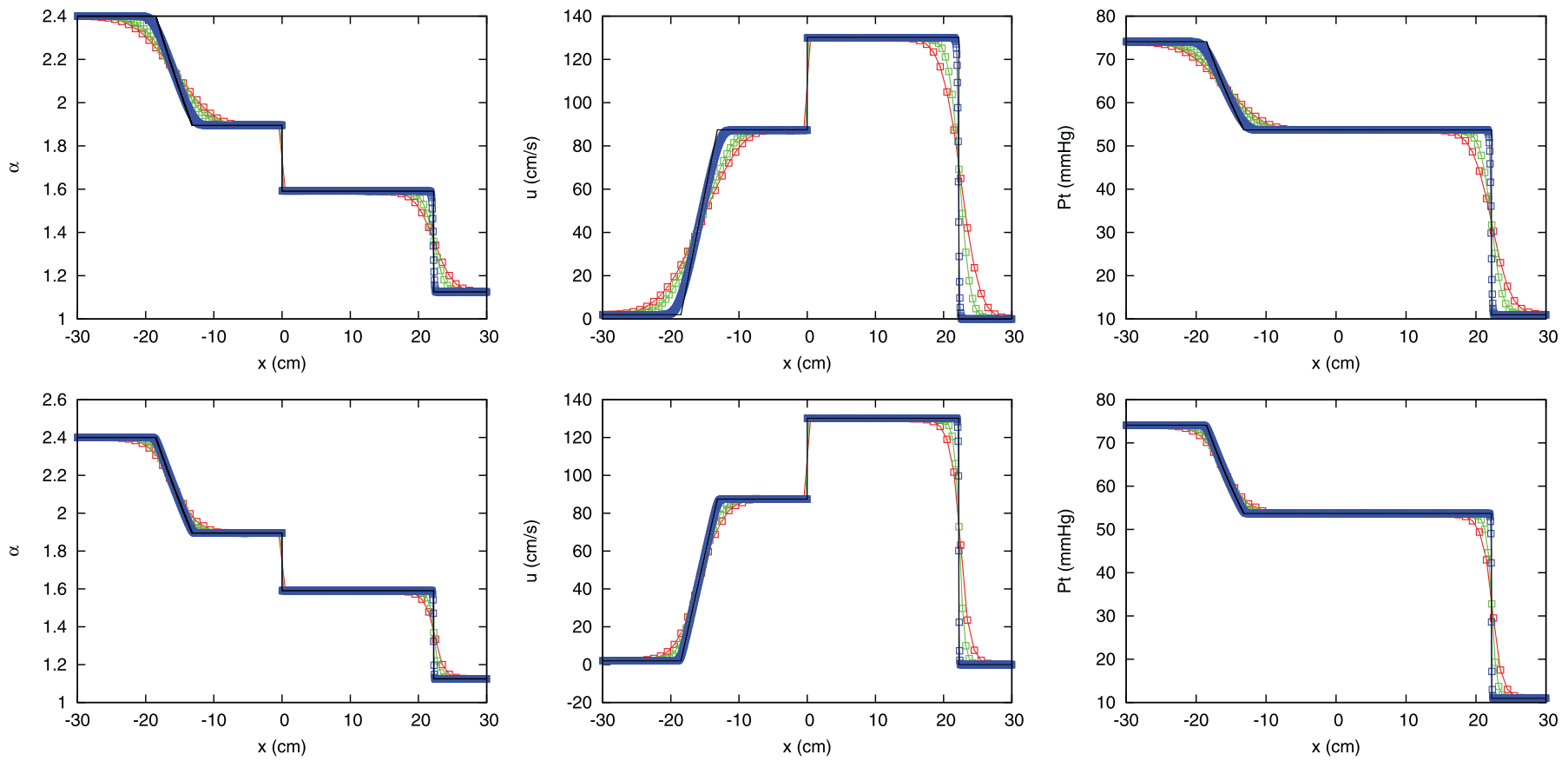

Fig. 4. Section 8.2. Test case 1. Subsonic RP in an artery. Comparison between exact (--) and numerical solutions at $t=0.05 \mathrm{~s}$ using the first order (upper) and TVDRK3 method (lower) using $\Delta x=1$ (red), 0.5 (green), 0.05 (blue) cm, for the HLLS solver $-\circ-$ and ARoe solver $-\square-$. (For interpretation of the references to colour in this figure legend, the reader is referred to the web version of this article.)
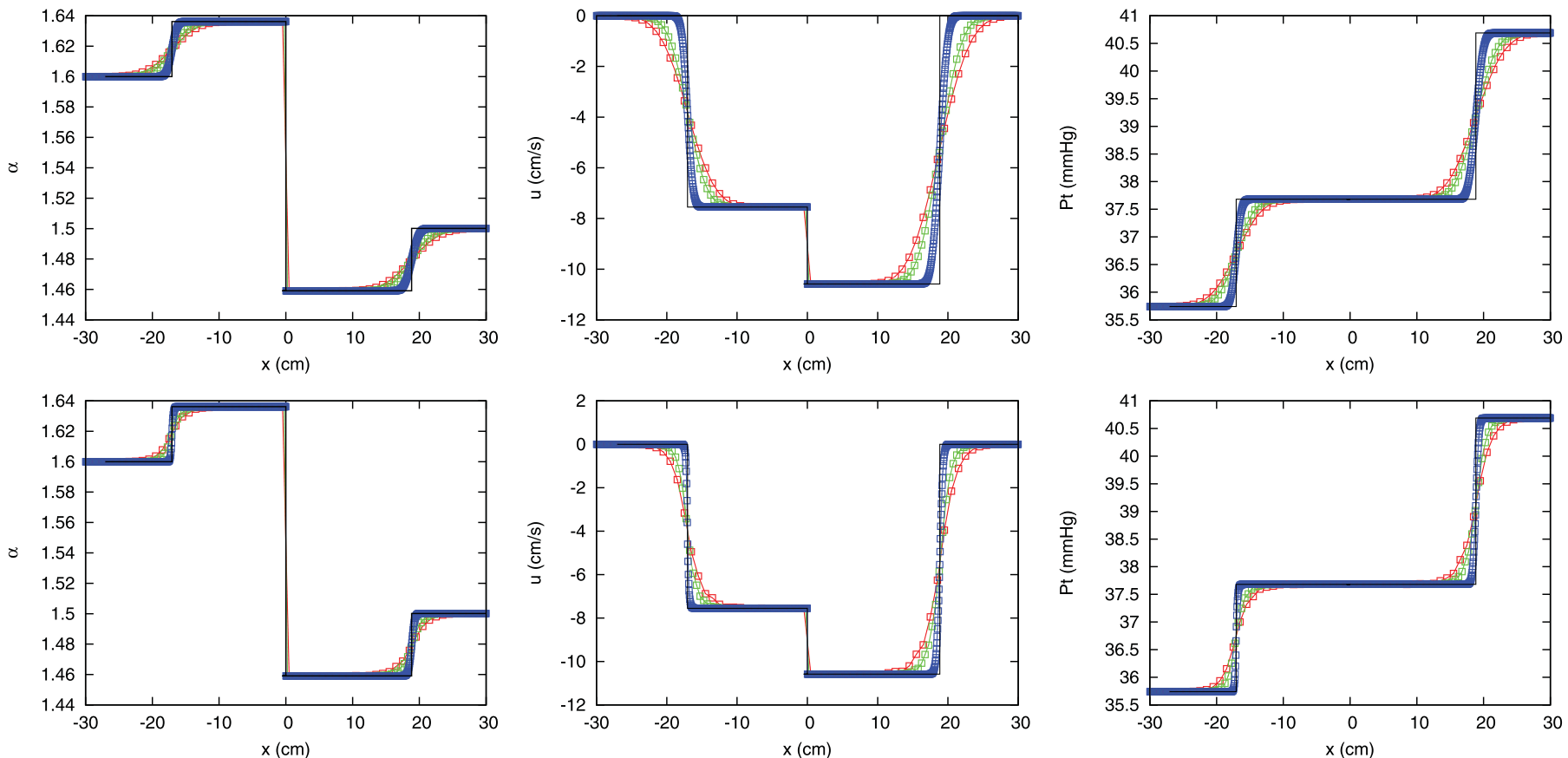

Fig. 5. Section 8.2. Test case 2. Subsonic RP in an artery. Comparison between exact (--) and numerical solutions at $t=0.05 \mathrm{~s}$ using the first order (upper) and TVDRK3 method (lower) using $\Delta x=1$ (red), 0.5 (green), 0.05 (blue) cm, for the HLLS solver - - and ARoe solver $-\square-$. (For interpretation of the references to colour in this figure legend, the reader is referred to the web version of this article.)

Supersonic test case 5 in Fig. 8 includes a stationary contact wave, a right moving elastic jump and a right moving rarefaction. When using both solvers the numerical diffusion decreases with grid refinement and with the extension from first to third order.

Test case 6 is a subsonic RP composed by left moving rarefaction, a stationary contact discontinuity and a left moving elastic jump where flow moves in the downstream direction. Even though veins have a much more non linear behavior than arteries, both numerical schemes capture correctly the solution and the high- order extension of the HLLS and ARoe solver preserves all the good features of their first-order versions, as shown in Fig. 9.

Test case 7 is a subsonic RP where flow moves initially in the downstream direction, but energy conditions force an upstream flow and, in consequence, a right moving elastic jump and a left moving rarefaction. Again predictions provided by the ARoe and HLLS solvers are indistinguishable for the three levels of mesh refinement (Fig. 10). Accurate results are obtained again for subsonic test case 8 in Fig. 11, composed by a left-moving elastic 

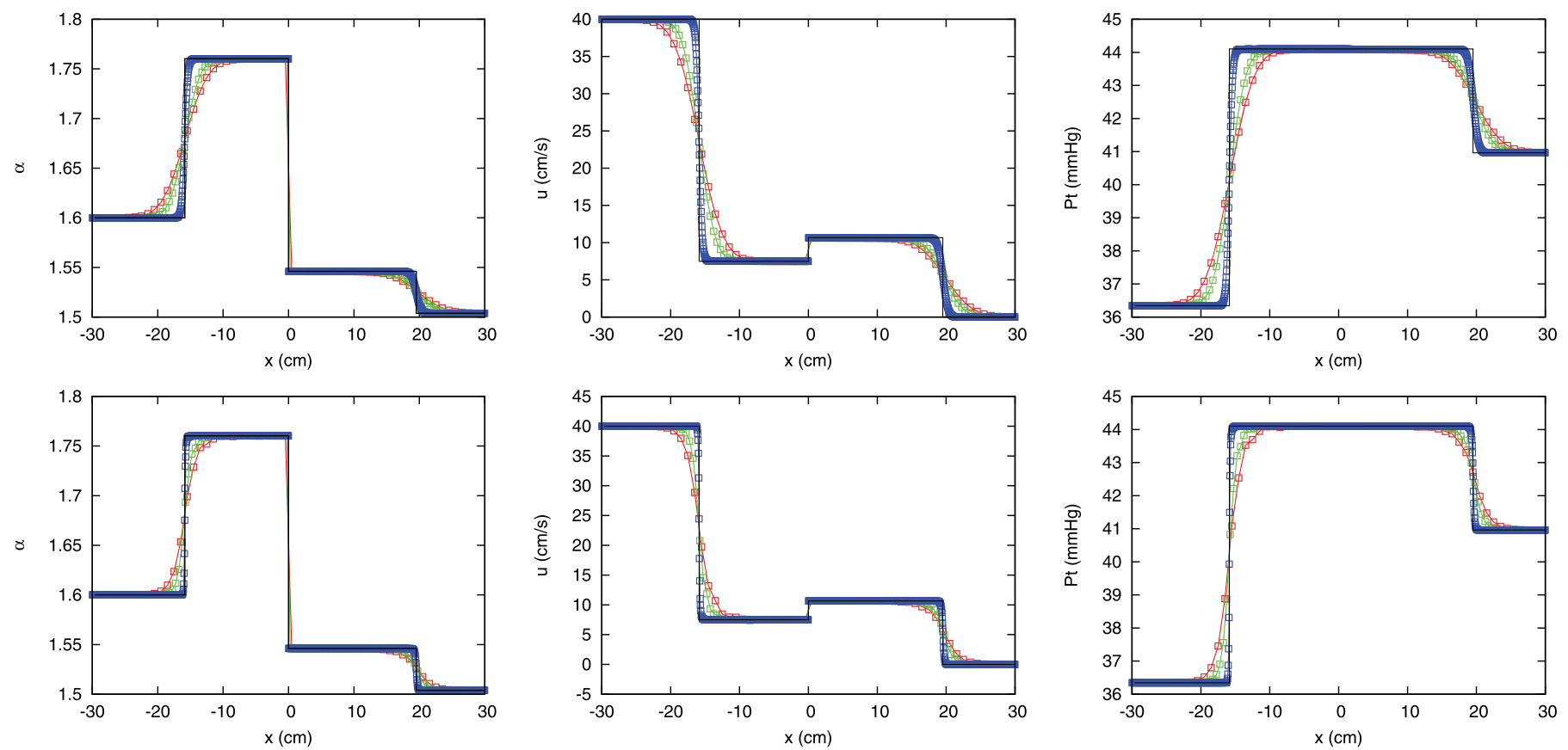

Fig. 6. Section 8.2. Test case 3. Subsonic RP in an artery. Comparison between exact (--) and numerical solutions at $t=0.05 \mathrm{~s}$ using the first order (upper) and TVDRK3 method (lower) using $\Delta x=1$ (red), 0.5 (green), 0.05 (blue) cm, for the HLLS solver - - and ARoe solver $-\square-$. (For interpretation of the references to colour in this figure legend, the reader is referred to the web version of this article.)
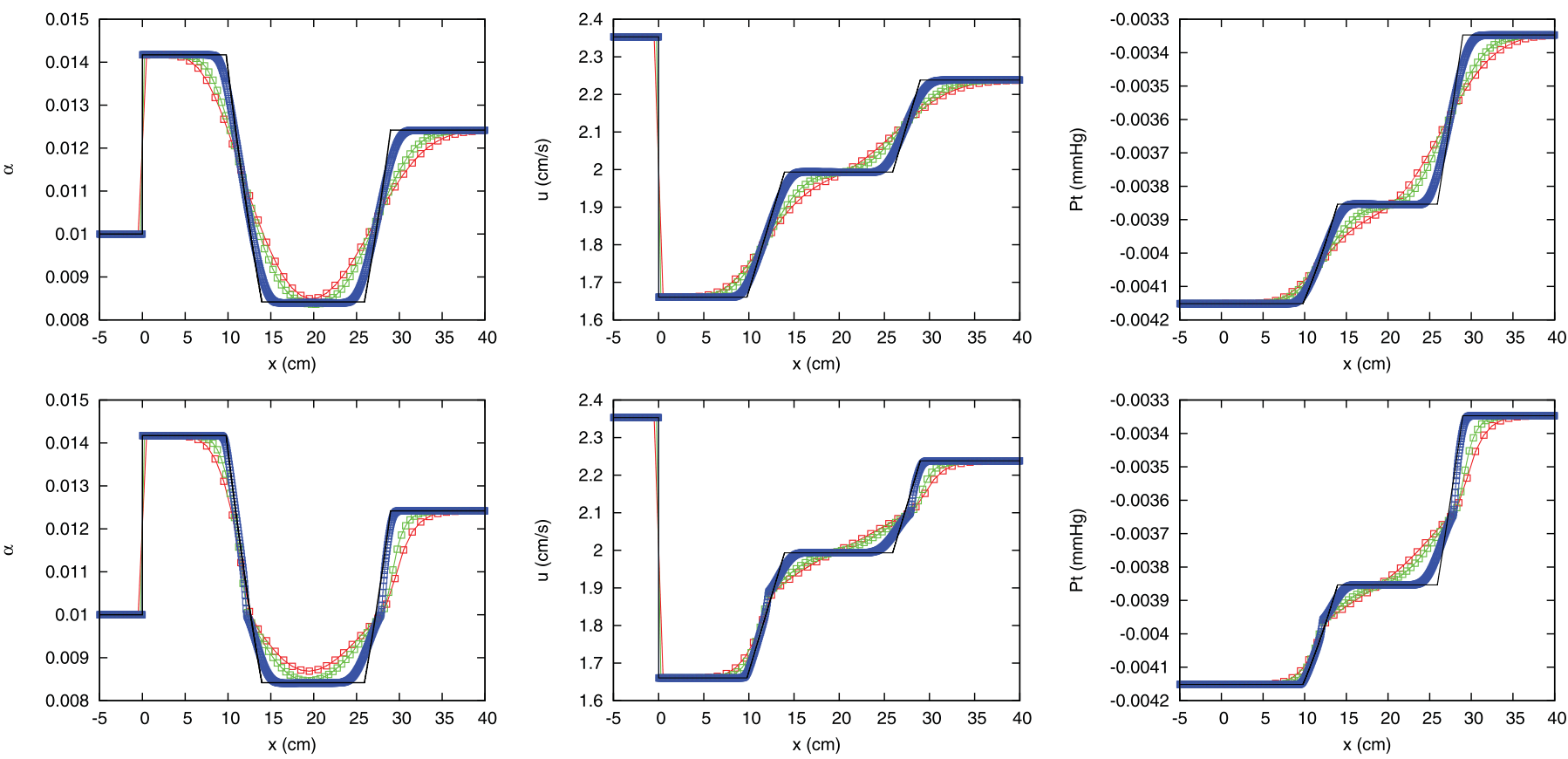

Fig. 7. Section 8.2. Test case 4. Supersonic RP in an artery. Comparison between exact (--) and numerical solutions at $t=10.0$ s using the first order (upper) and TVDRK3 method (lower) using $\Delta x=1$ (red), 0.5 (green), 0.05 (blue) cm, for the HLLS solver - - and ARoe solver $-\square-$. (For interpretation of the references to colour in this figure legend, the reader is referred to the web version of this article.)

jump, a stationary contact wave at the discontinuity and a rightmoving elastic jump wave. It is worth mentioning that a mild undershoot of area and velocity profiles at the onset of the rarefaction wave, $x \approx 32$ are observed. The behavior seems to match that observed when using other different numerical schemes, as high order path-conservative schemes [20] and TVD shock-capturing methods [30]. In our experience this discrepancies is related to the non-linear nature of the characteristic field generating the rarefaction wave. We have observed also this behavior in the SWE, when using energy balanced WENO-ADER solvers [22]. Although less pronounced, the same effect can be observed in test case 6 at $x \approx-22$.

In test case 9, Fig. 12, the solution includes a left-moving rarefaction wave, a stationary contact wave at the discontinuity and a right-moving rarefaction wave. Again, the high-order version of both schemes preserves all the good features of the first-order HLLS and ARoe schemes, such as the energy-balanced property. Also, there is an improvement of the resolution of both, contact 

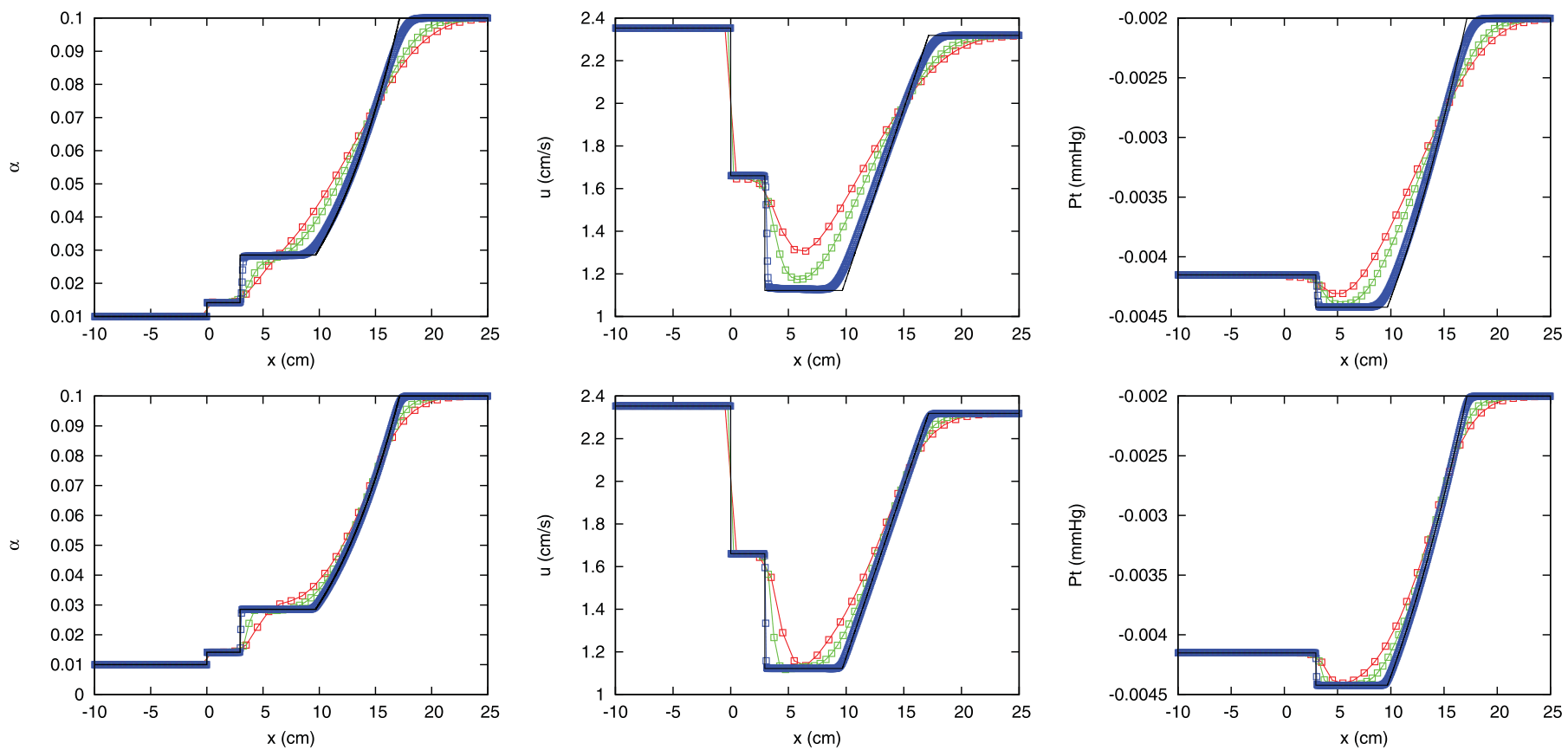

Fig. 8. Section 8.2. Test case 5. Supersonic RP in an artery. Comparison between exact (--) and numerical solutions at $t=5.0$ s using the first order (upper) and TVDRK3 method (lower) using $\Delta x=1$ (red), 0.5 (green), 0.05 (blue) cm, for the HLLS solver - - and ARoe solver $-\square-$. (For interpretation of the references to colour in this figure legend, the reader is referred to the web version of this article.)
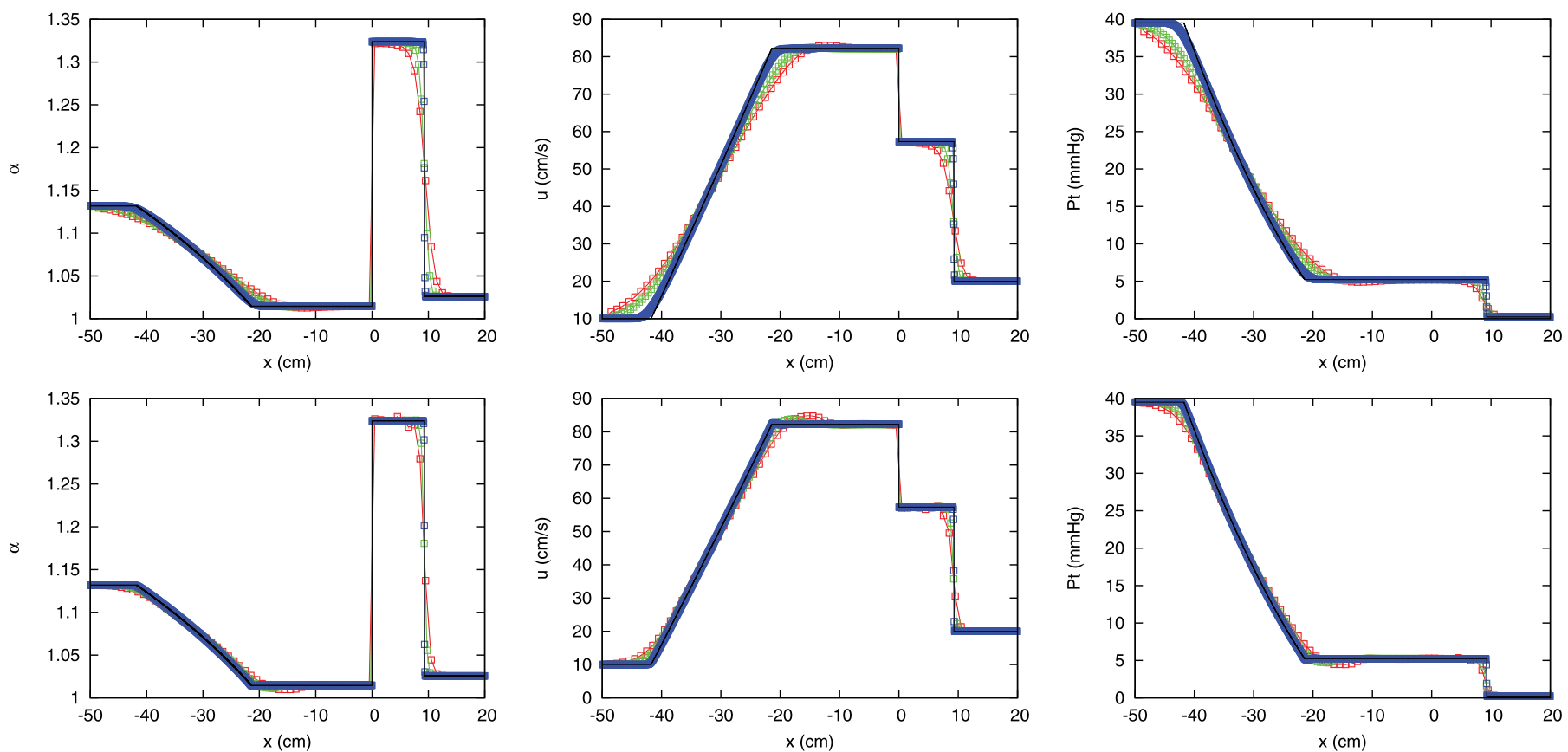

Fig. 9. Section 8.2. Test case 6. Subsonic RP in a vein. Comparison between exact (--) and numerical solutions at $t=0.05$ s using the first order (upper) and TVDRK3 method (lower) using $\Delta x=1$ (red), 0.5 (green), 0.05 (blue) cm, for the HLLS solver - - and ARoe solver $-\square-$. (For interpretation of the references to colour in this figure legend, the reader is referred to the web version of this article.)

wave and rarefactions, when comparing first-order and high order results.

Table 4 shows the time steps $\Delta t$ computed using (96) for both HLLS and ARoe scheme and for test cases 1 to 9 setting $\Delta x=1$, using first order approximation. The time step remains almost constant along the simulation of each RP and is approximately equal for both solvers. Both schemes behave in the same way when using the third order approximation.

\subsection{Collapsed vessels in riemann problems with exact solutions.}

In this section the performance of the numerical methods is checked using RP's involving vessel collapse. Initial conditions, geometrical and mechanical properties are listed in Table 5.

Test case 10 is a supersonic flow between two vessels with variable mechanical properties in an artery. The solution in Fig. 13 shows that, after the stationary contact discontinuity generated by 

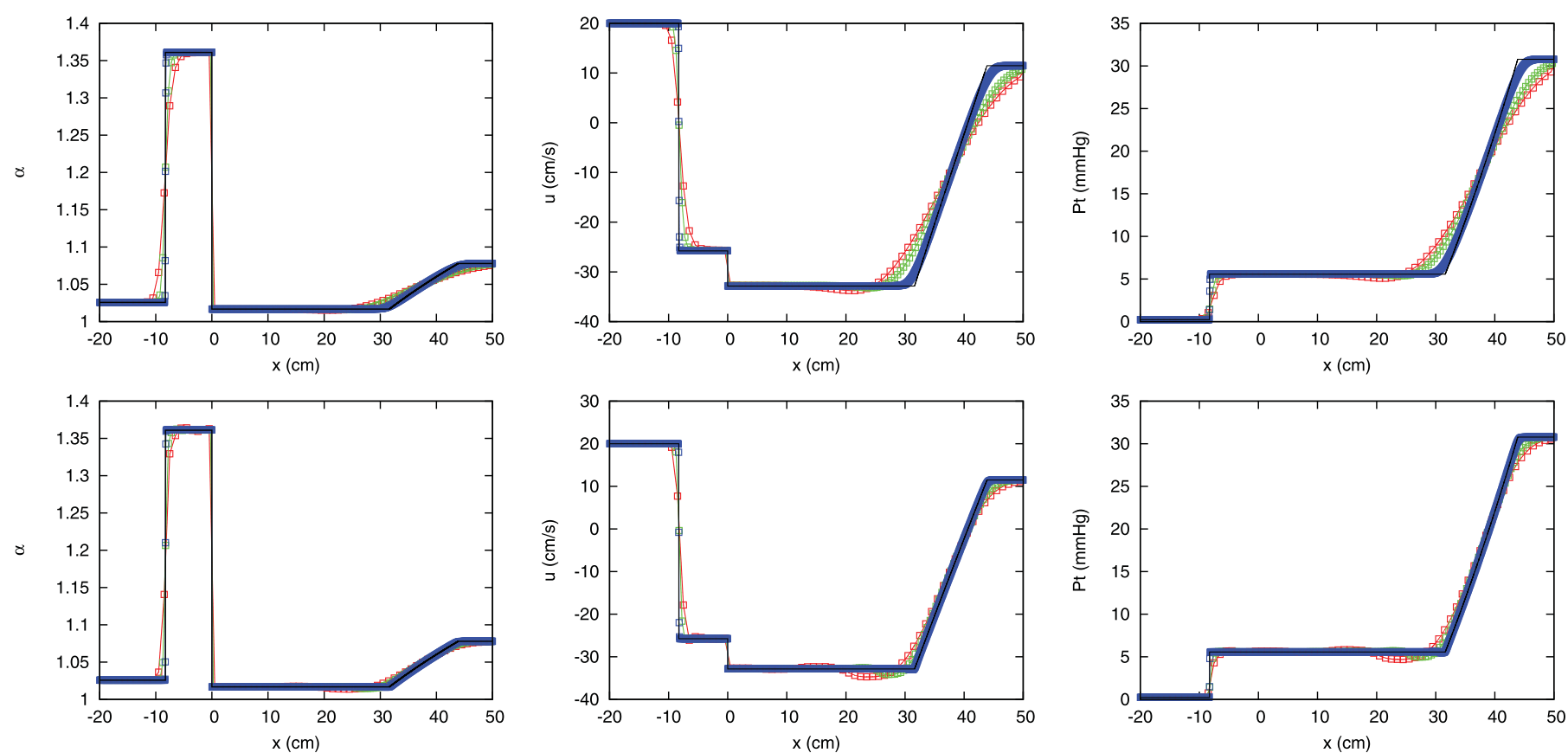

Fig. 10. Section 8.2. Test case 7. Subsonic RP in a vein. Comparison between exact (--) and numerical solutions at $t=0.05 \mathrm{~s}$ using the first order (upper) and TVDRK3 method (lower) using $\Delta x=1$ (red), 0.5 (green), 0.05 (blue) cm, for the HLLS solver - $\circ$ - and ARoe solver $-\square-$. (For interpretation of the references to colour in this figure legend, the reader is referred to the web version of this article.)
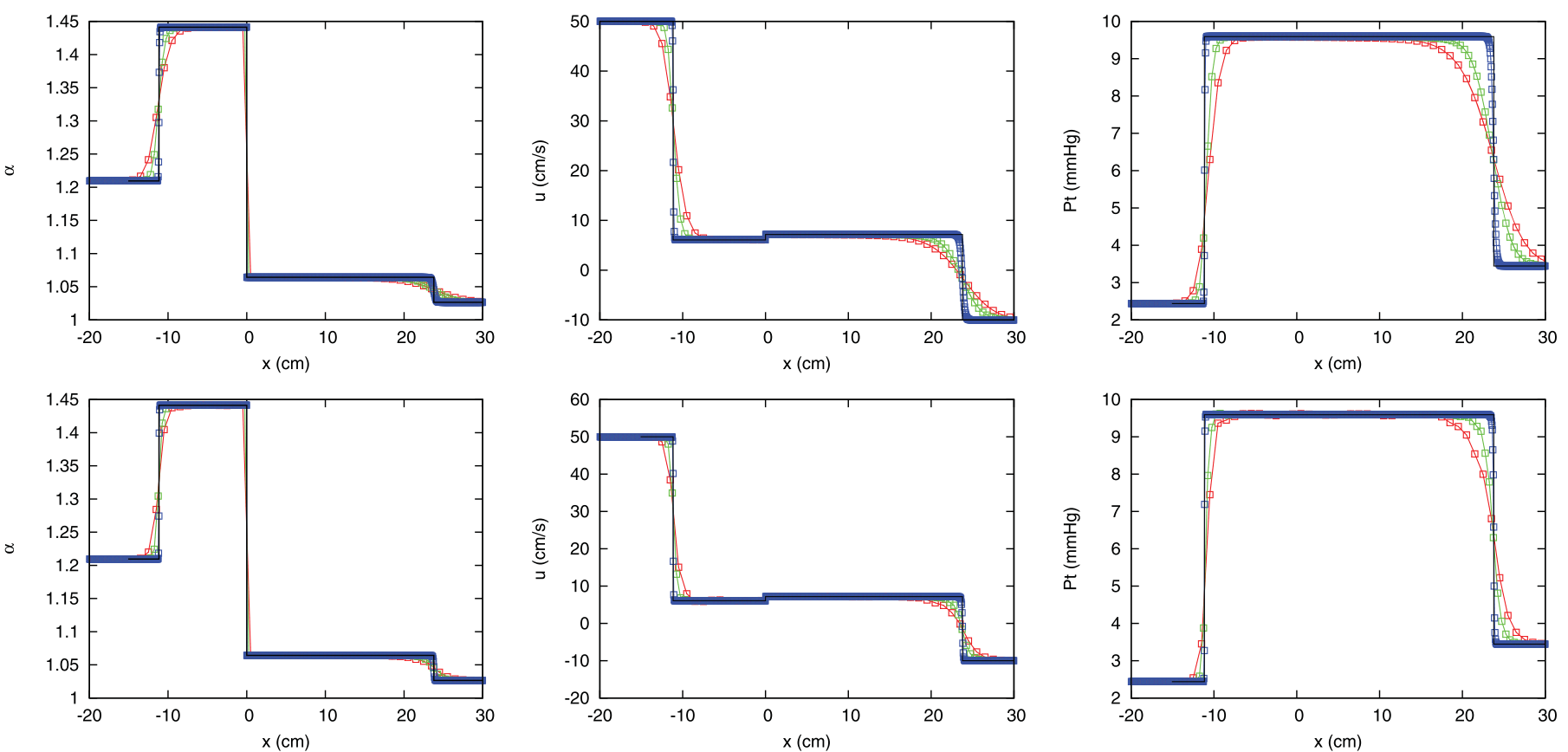

Fig. 11. Section 8.2. Test case 8. Subsonic RP in a vein. Comparison between exact (--) and numerical solutions at $t=0.05 \mathrm{~s}$ using the first order (upper) and TVDRK3 method (lower) using $\Delta x=1$ (red), 0.5 (green), 0.05 (blue) cm, for the HLLS solver - $\circ$ - and ARoe solver $-\square-$. (For interpretation of the references to colour in this figure legend, the reader is referred to the web version of this article.)

Table 4

Time step $\Delta t(\mathrm{~s})$ for each RP with $\Delta x=1$.

\begin{tabular}{llllllllll}
\hline RP & 1 & 2 & 3 & 4 & 5 & 6 & 7 & 8 & 9 \\
\hline ARoe & 0.00087 & 0.00115 & 0.00113 & 0.00053 & 0.00051 & 0.00086 & 0.00107 & 0.14827 & 0.13120 \\
HLLS & 0.00087 & 0.00115 & 0.00113 & 0.00053 & 0.00051 & 0.00086 & 0.00107 & 0.14827 & 0.13120 \\
\hline
\end{tabular}



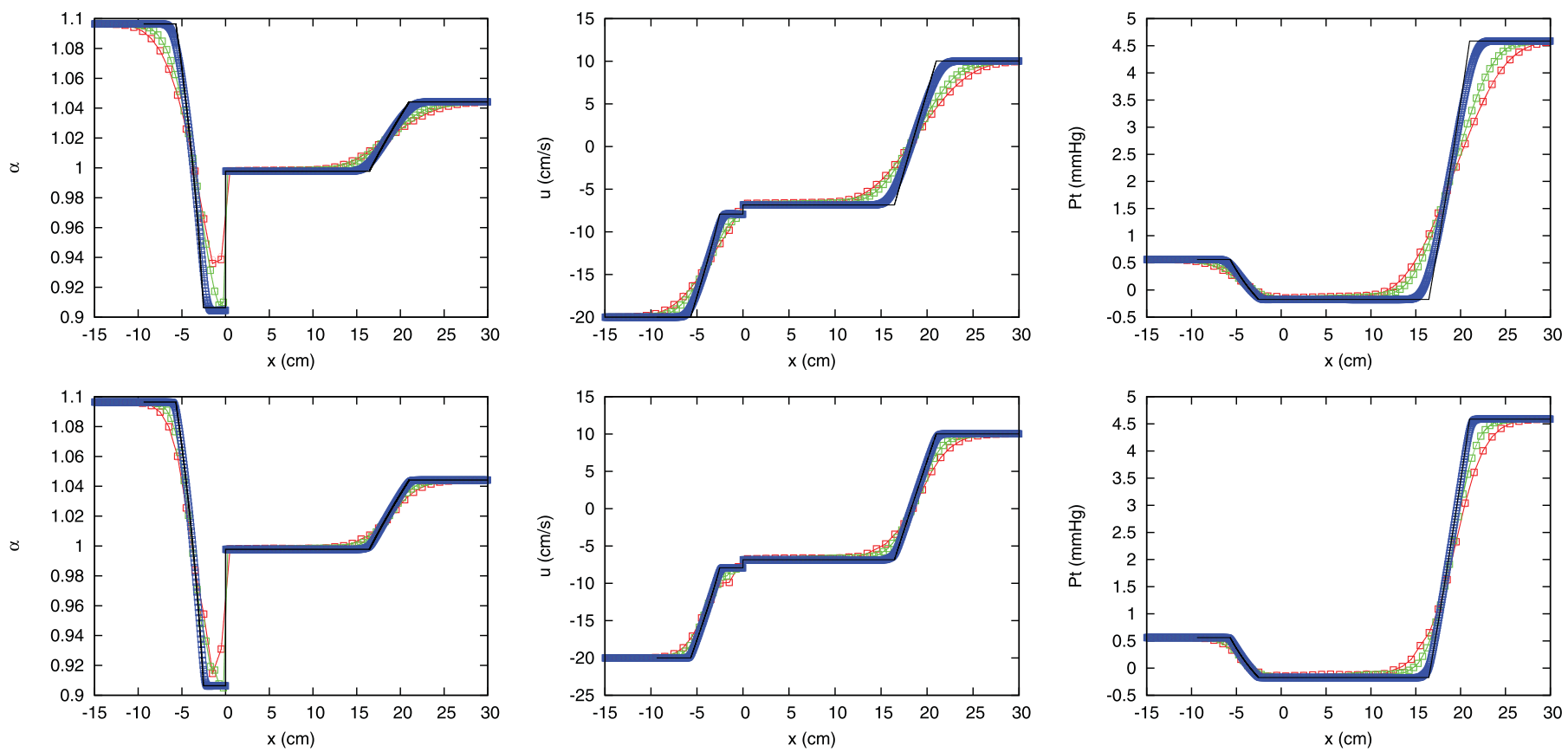

Fig. 12. Section 8.2. Test case 9. Subsonic RP in a vein. Comparison between exact (--) and numerical solutions at $t=0.05$ s using the first order (upper) and TVDRK3 method (lower) using $\Delta x=1$ (red), 0.5 (green), 0.05 (blue) cm, for the HLLS solver - $\circ$ - and ARoe solver $-\square-$. (For interpretation of the references to colour in this figure legend, the reader is referred to the web version of this article.)
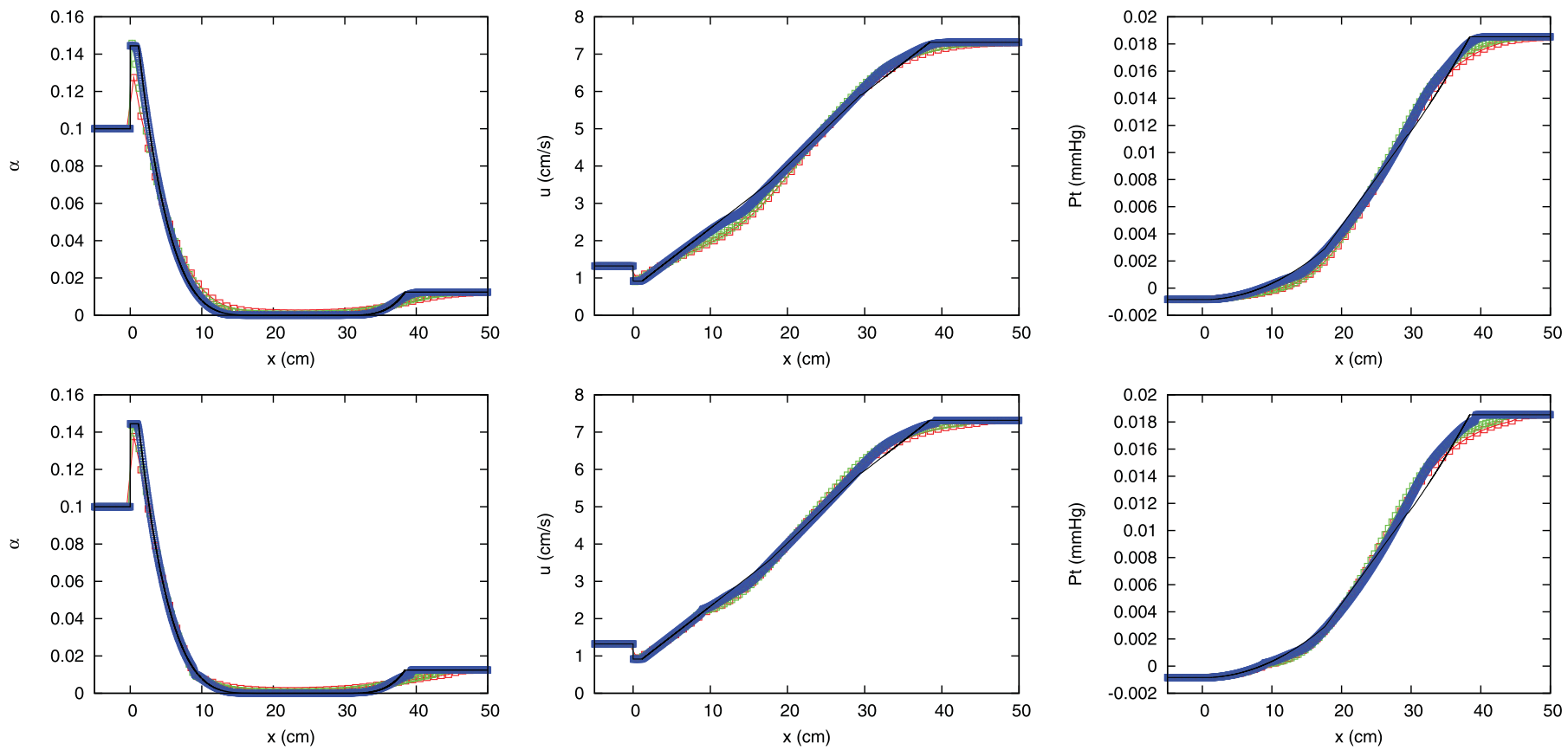

Fig. 13. Section 8.4. Test case 10. Supersonic collapsed RP in an artery. Comparison between exact (--) and numerical solutions at $t=5.0$ s using the first order (upper) and TVDRK3 method (lower) using $\Delta x=1$ (red), 0.5 (green), 0.05 (blue) cm, for the HLLS solver - - - and ARoe solver $-\square-$. (For interpretation of the references to colour in this figure legend, the reader is referred to the web version of this article.) 

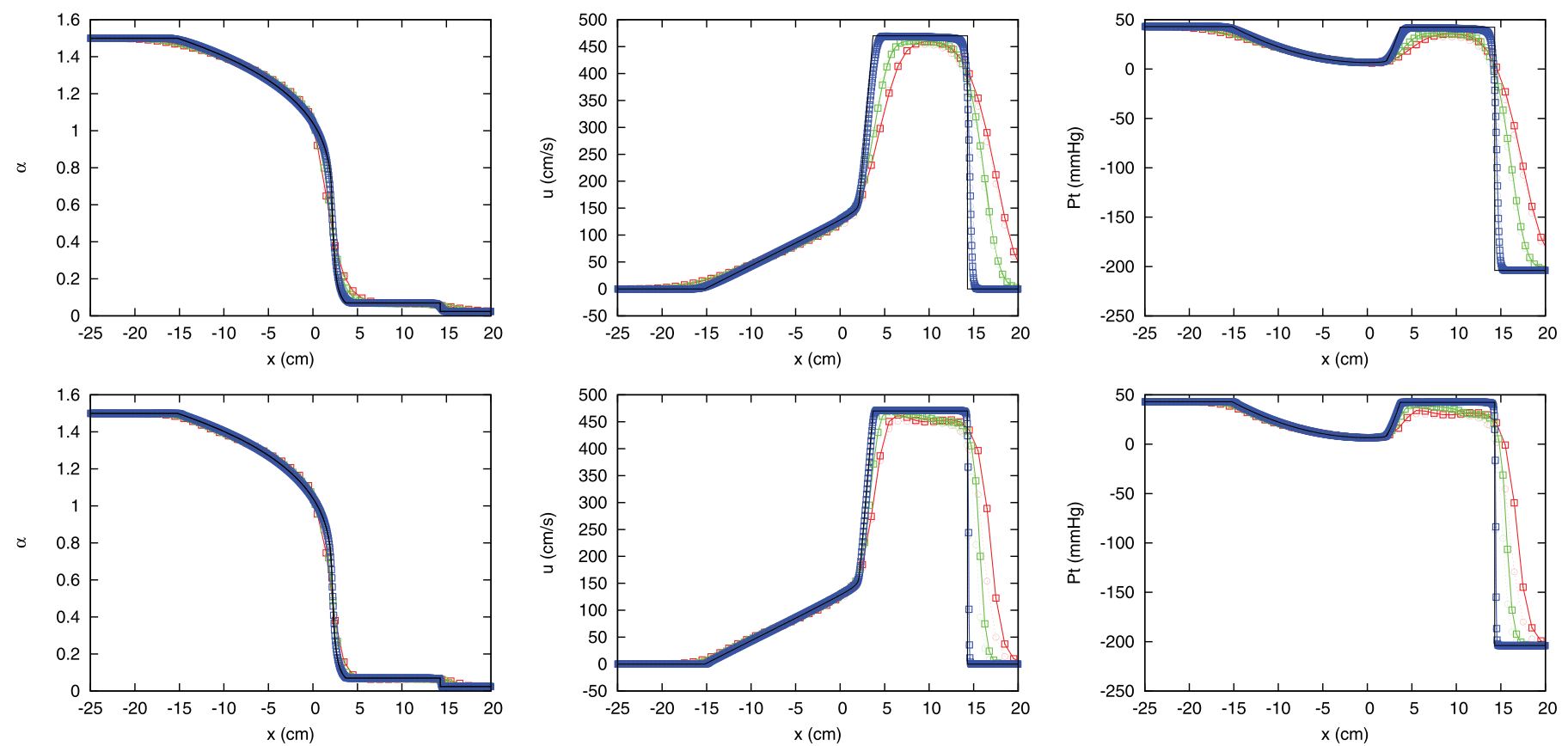

Fig. 14. Section 8.4. Test case 11. Subsonic-supersonic collapsed RP in a vein. Comparison between exact (--) and numerical solutions at $t=0.02 \mathrm{~s}$ using the first order (upper) and TVDRK3 method (lower) using $\Delta x=1$ (red), 0.5 (green), 0.05 (blue) cm, for the HLLS solver - - and ARoe solver $-\square-$. (For interpretation of the references to colour in this figure legend, the reader is referred to the web version of this article.)

Table 5

Left and right mechanical and geometrical properties and initial conditions.

\begin{tabular}{lllllll}
\hline Test & $A_{L, o}\left(\mathrm{~cm}^{2}\right)$ & $K_{L}(\mathrm{~Pa})$ & $A_{L}\left(\mathrm{~cm}^{2}\right)$ & $u_{L}(\mathrm{~m} / \mathrm{s})$ & $m$ & $n$ \\
\hline 10 & 1.0 & 0.290682 & 0.1 & 1.323213 & 0.5 & 0 \\
11 & 0.031415927 & 100.0 & 0.04712389 & 0.0 & 10 & -1.5 \\
12 & 0.3 & 0.001 & 0.3 & -10.0 & 10 & -1.5 \\
13 & 0.031415927 & 0.00111 & 0.067230083 & -30.0 & 10 & -1.5 \\
Test & $A_{R, 0}$ & $K_{R}$ & $A_{R}$ & $u_{R}$ & $m$ & $n$ \\
10 & 1.0 & 0.247 & 0.012429978 & 7.3158749 & 0.5 & 0 \\
11 & 0.031415927 & 100.0 & 0.000751391 & 0.0 & 10 & -1.5 \\
12 & 0.2 & 0.100 & 0.3 & 12.969478 & 10 & -1.5 \\
13 & 0.031415927 & 0.00111 & 0.067230083 & 30.0 & 10 & -1.5 \\
\hline
\end{tabular}

the change in elasticity, a right moving rarefaction wave develops leading to an extreme reduction of the vessel area. The velocity changes linearly while the area is recovered along the left moving rarefaction wave. The numerical solutions converge to the exact solution preserving a correct value of total energy at the initial discontinuity and at both rarefactions. ARoe and HLLS schemes provide accurate and indistinguishable results for both first and third order.

A RP involving a collapsed vein is defined in test case 11, with continuous geometrical and mechanical properties. The vein is initially collapsed in the right side of the RP, leading to a supersonic flow that generates a left moving rarefaction wave and a left moving elastic jump (Fig. 14). Numerical results provided by the ARoe and the HLLS schemes are accurate for both first and third order and converge to the exact solution, being the HLLS less diffusive.

Test case 12 is a very complex case. Collapse is forced by two opposite expansion fans communicated by an stationary contact discontinuity generated by the change in geometrical and mechanical properties of the vessel. Differences between solvers appear. Exact and numerical solutions for first order approximation are shown in Figs. 15 and 16. Numerical solutions for the dimensionless area reference $\alpha$ converge to the exact solution with mesh refinement. Noticeable differences among numerical and exact solutions are observed in velocity and total energy.
Fig. 16 shows numerical results for both solvers for speed index, pulse wave celerity $c$ and flow $Q$. The pulse wave celerity does not have a monotonous behavior and does not decrease continuously with vessel area reduction. There is a critical area value where the speed of the pulse wave is minimal and, from this point, an additional reduction of area causes the velocity of the pulse wave to grow again. As a consequence, the speed index first increases and, once this critical value is exceeded, decreases again. Even though the results for vessel area are accurate, the differences in the total energy and pulse wave celerity are noticeable in the collapsed region. The reason is that as in this region the relation between area and pressure or pulse wave celerity are highly non linear. On the other hand, satisfactory results are provided for the conserved variable $Q$. This conserved variable is less sensitive to the dramatic reduction of vessel area produced in this case. In general, the HLLS provides better results than the ARoe solver for both first and also third order, as shown in Fig. 17.

Continuous geometrical and mechanical properties are defined in the collapsing test case 13, plotted in Fig. 18, less stiff than test case 12 . The solution consists of two opposite rarefaction fans connected by an extremely collapsed area. Numerical results of the first order HLLS are plotted using first order (upper) and third order (middle and lower) approximations including the speed index, wave pulse celerity and flood discharge. Only the HLLS is able to provide a numerical solution. Again, speed index suffers strong variations in the vicinity of the collapsed area that can not be managed by the ARoe solver. The position of the minimal pulse wave area celerity and maximum speed index is well captured and accurate results are provided for conserved variable $Q$. Numerical results show very good agreement with the exact solution and improve with mesh refinement and the proposed high order extension. The failure of the ARoe scheme in this particular case may be explained by the rigidity of the solver when defining the approximate wave celerities. The HLLS solver merges the rich description of the inner states given by the ARoe solver with the flexibility in the selection of the wave celerities of the system, providing a more robust numerical scheme. 

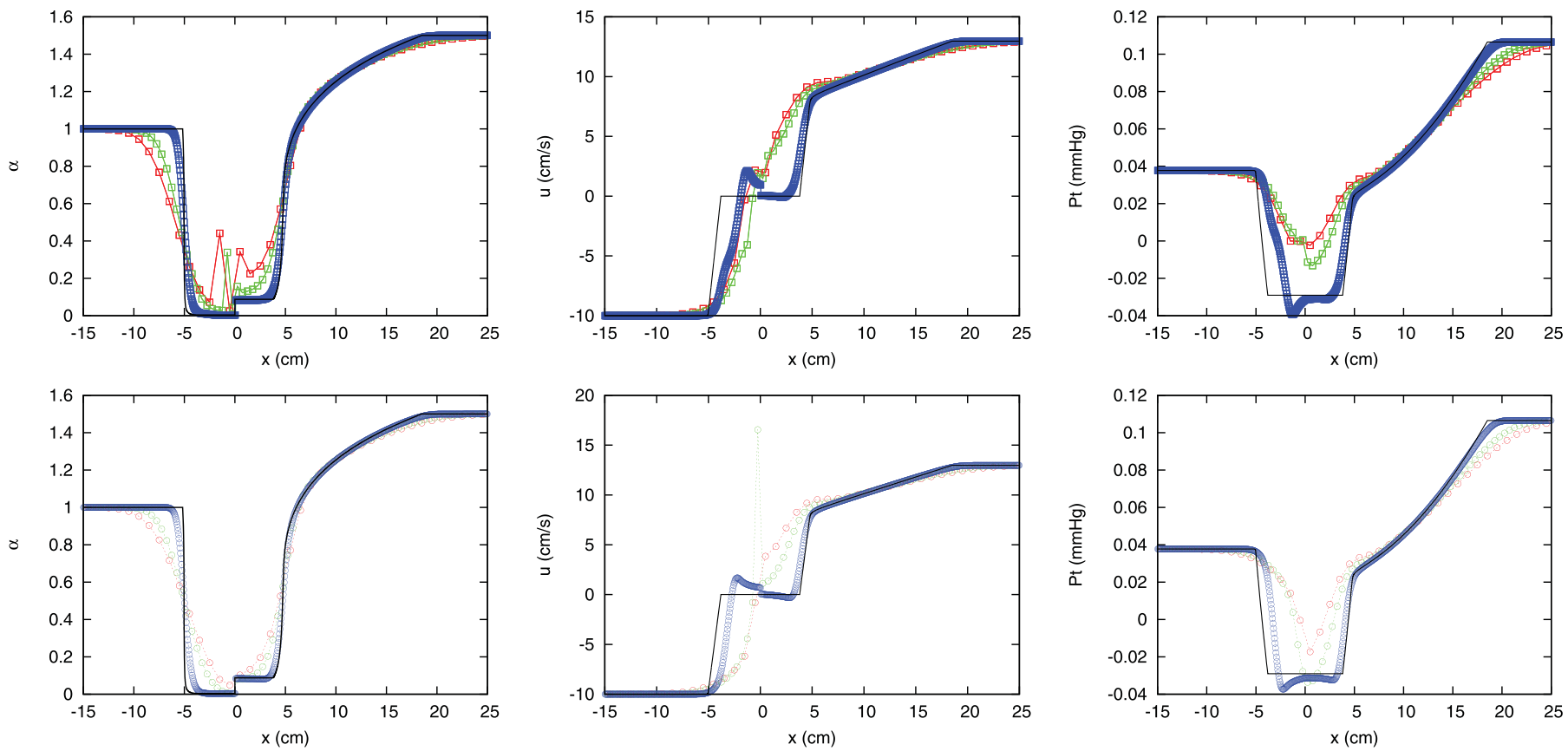

Fig. 15. Section 8.4. Test case 12. Subsonic-supersonic collapsed RP in a vein. Exact (--) and numerical solutions at $t=0.5 \mathrm{~s}$ for $\Delta x=1$ (red), 0.5 (green), 0.05 (blue) $\mathrm{cm}$, for the first order ARoe solver $-\square-$ and the first order HLLS solver - -- . (For interpretation of the references to colour in this figure legend, the reader is referred to the web version of this article.)
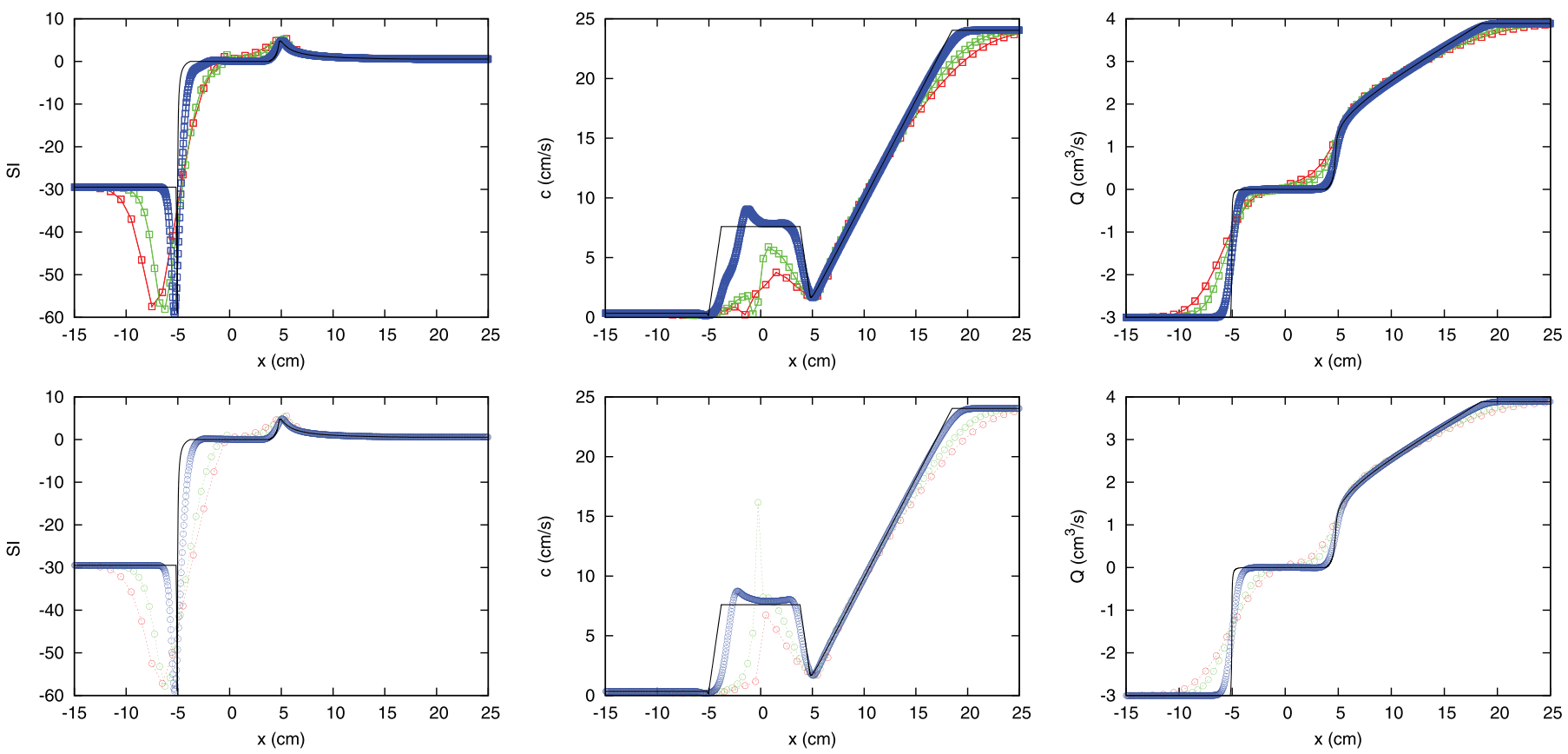

Fig. 16. Section 8.4. Test case 12. Subsonic-supersonic collapsed RP in a vein. Exact (--) and numerical solutions at $t=0.5 \mathrm{~s}$ for $\Delta x=1$ (red), 0.5 (green), 0.05 (blue) $\mathrm{cm}$, for the first order ARoe solver $-\square-$ and the first order HLLS solver - - -. (For interpretation of the references to colour in this figure legend, the reader is referred to the web version of this article.) 

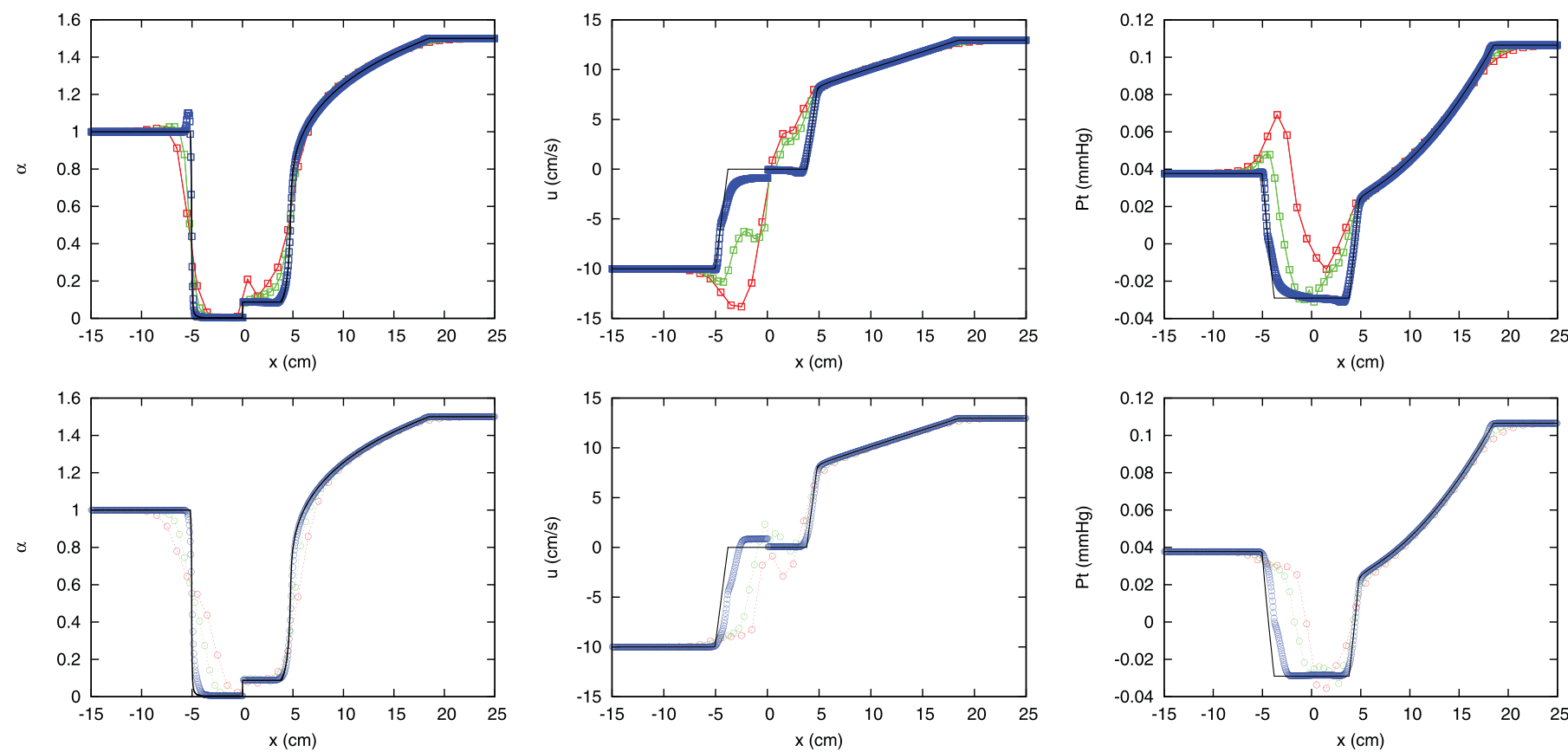

Fig. 17. Section 8.4. Test case 12. Subsonic-supersonic collapsed RP in a vein. Exact (--) and numerical solutions at $t=0.5 \mathrm{~s}$ for $\Delta x=1$ (red), 0.5 (green), 0.05 (blue) $\mathrm{cm}$, for the TVDRK3 ARoe solver $-\square-$ and the TVDRK3 HLLS solver - - -. (For interpretation of the references to colour in this figure legend, the reader is referred to the web version of this article.)

Table 6

Time step $\Delta t$ (s) for RP's 10 to 13 with $\Delta x=1$.

\begin{tabular}{lllll}
\hline RP & 10 & 11 & 12 & 13 \\
\hline ARoe & 0.05854 & 0.00010 & 0.01216 & - \\
HLLS & 0.05854 & 0.01001 & 0.01216 & 0.00058
\end{tabular}

Table 7

Left and right mechanical and geometrical properties and initial conditions in RP's 14 to 17.

\begin{tabular}{llllll}
\hline$A_{L, o}\left(\mathrm{~cm}^{2}\right)$ & $K_{L}(\mathrm{~Pa})$ & $A_{L}\left(\mathrm{~cm}^{2}\right)$ & $u_{L}(\mathrm{~m} / \mathrm{s})$ & $m$ & $n$ \\
2.0 & 100.0 & 2.0 & 0.0 & 10 & -1.5 \\
$A_{R, o}$ & $K_{R}$ & $A_{R}$ & $u_{R}$ & $m$ & $n$ \\
2.0 & 100.0 & 2.0 & 0.0 & 10 & -1.5 \\
\hline
\end{tabular}

Table 6 shows the minimum time steps $\Delta t$ computed using (96) for both HLLS and ARoe scheme and for test cases 1 to 9 setting $\Delta x=1$, using first order approximation. The time step remains almost constant along the simulation of each RP when using the HLLS scheme. For the ARoe scheme, time step is strongly reduced in RP 11, confirming the diffusive behavior of the ARoe scheme in this case. For test cases 10 and 12 the time step is nearly constant an approximately equal for both solvers.

\subsection{Riemann problems under expansion conditions.}

In this set of RPs, the transition from subsonic to sonic blockage conditions is analyzed. Left and right mechanical and geometrical properties and initial conditions in Table 7 are the same in all cases except for the variation in external pressure, atmospheric on the left side and sub-atmospheric on the right side, defined in Table 8. Pressure variation in test case 14 leads to a subsonic flow defined by a left moving rarefaction wave that leads to a progressive narrowing of the vessel area in the contact discontinuity followed by an abrupt expansion on the side with sub-atmospheric pressure. In Fig. 19, it is shown that both the HLLS and the a ARoe solver
Table 8

Sub-atmospheric conditions in RP's 14 to 17 .

\begin{tabular}{lll}
\hline Test & $p_{L, e}(\mathrm{mmHg})$ & $p_{R, e}(\mathrm{mmHg})$ \\
\hline 14 & 0.0 & -4.0 \\
15 & 0.0 & -4.9 \\
16 & 0.0 & -10.0 \\
17 & 0.0 & -20.0 \\
18 & 0.0 & -40.0 \\
\hline
\end{tabular}

provide accurate and indistinguishable results with independence of the order of the scheme. Note that in this case, energy is kept constant across the sudden change in vessel area.

In test case 15 suction on the left side of the RP is increased with respect to the previous case and sonic conditions are forced across the contact discontinuity. The flow generated by the left rarefaction leads to a critical vessel area in the narrowest section were speed index is defined by the sonic condition and therefore sonic blockage appears. The comparison of results for this test case is shown in Fig. 20. Both first and third orde schemes provide good agreement with the exact solution for the equivalent nozzle throat and the expanded area. Numerical differences between the results given by the HLLS and the ARoe solvers are indistinguishable. Fig. 21 shows comparison of results for total energy $P_{t}$ in test cases (left) 14 and (right) 15. In both test cases, the numerical solutions ensure the conservation of the discrete level of energy across the contact discontinuity.

Numerical results for $\alpha$, SI and total energy in test cases 16 to 18 are shown in Figs. 22, 23 and 24 respectively. These numerical experiments have been conducted to analyze the performance of the numerical solvers in cases involving an energy dissipation formulation while keeping the maximum flow provided by the sonic blockage condition and the minimum throat section. As sonic blockage has been already generated in test case 15 , despite the increase in suction in the right side of the RP in test 

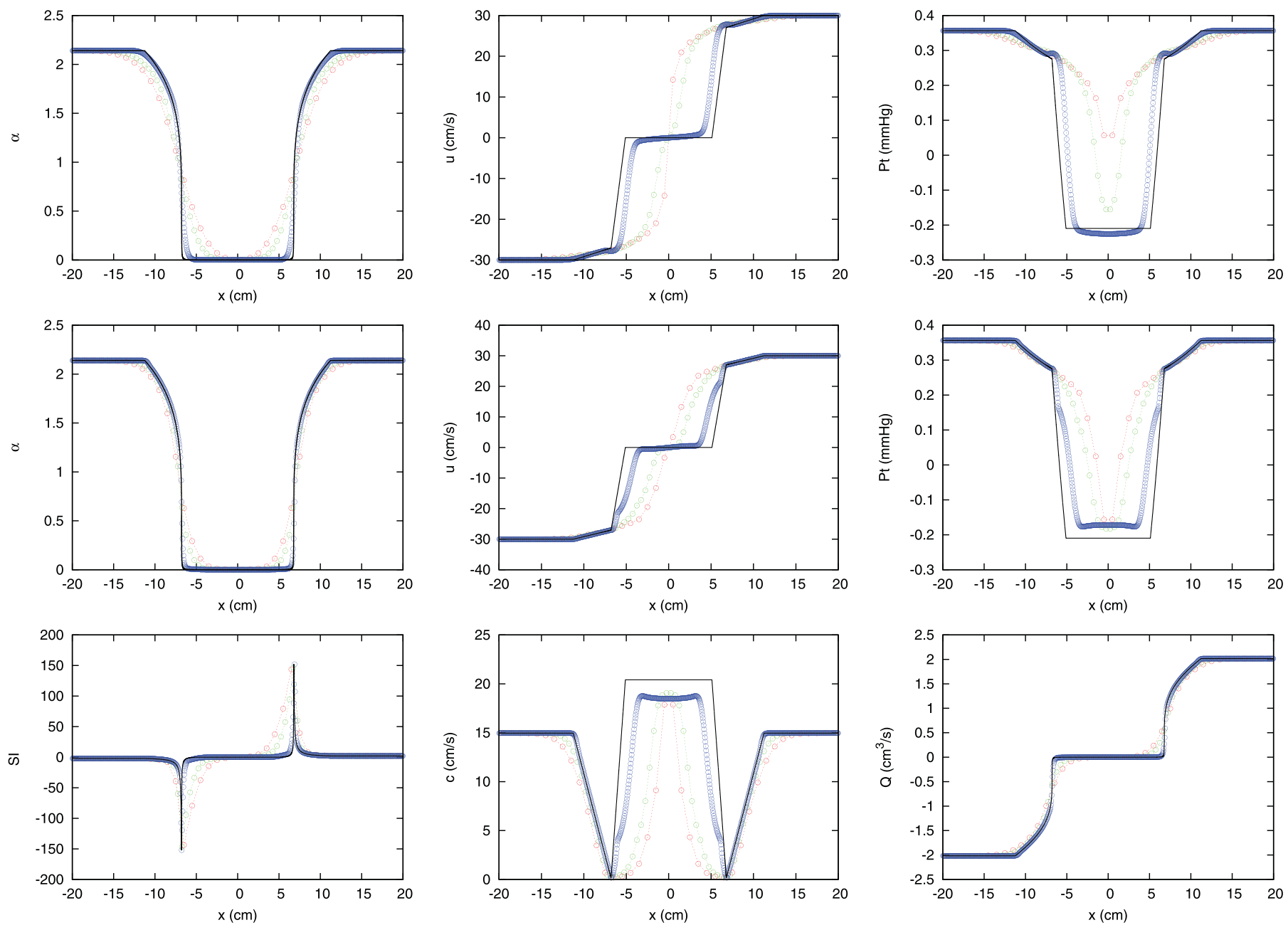

Fig. 18. Section 8.4. Test case 13. Subsonic-supersonic collapsed RP in a vein. Exact (--) and numerical solutions at $t=0.25 \mathrm{ss}$ for $\Delta x=1$ (red), 0.5 (green), 0.05 (blue) $\mathrm{cm}$, for the first order (upper) and third order (middle and lower) HLLS solver. (For interpretation of the references to colour in this figure legend, the reader is referred to the web version of this article.)

cases 16 to 18, all exact solutions must be identical. Propagation speed of the right-moving shock provided by the numerical solvers depends on the mesh spacing and converges to the exact solution with mesh refinement. We have noticed the same behavior in other non-homogenous hyperbolic problems where solvers are applied to systems expressed in conservative form [23].

Numerical solutions show how the numerical integration of the source terms does not allow further flow acceleration with an increasing values of suction. The sonic blockage condition is ensured in all cases. In a first order approximation, numerical solutions provided by the HLLS solver are more diffusive than those provided by the ARoe solver. Even though numerical differences between solvers decrease when using the third order extension of the numerical scheme, numerical oscillations around the discontinuity in total energy can be observed for the ARoe solver in Fig. 24. The third order HLLS solver is able to reproduce more accurately the intermediate state on the right hand side of the discontinuity.

When using first or third order approximations and $\Delta x=1 \mathrm{~cm}$, in RP's 14 to 18 the time step was nearly constant during each simulation. The value of $\Delta t$ was approximately equal to 0.0013 for RP 14 , and approximately equal to 0.0002 for RP 15 to 18 , for both solvers.

\section{Conclusions}

Well-balanced first and third-order numerical schemes for onedimensional flow in blood vessels with variable mechanical and geometrical properties including collapsed states are presented. HLLS and ARoe flux solvers have been described in flux and fluctuation form by transforming the initial RP formulation in an approximate RP written in conservative-law form.

The well-balanced property of the numerical schemes have been verified. Numerical flux solvers and their corresponding entropy and positivity fixes have been systematically assessed and validated in a wide variety of RP's, involving variations in mechanical and geometrical properties, subsonic and supersonic cases, energy conservation across contact discontinuities, flow limitation and extreme collapse. Pulse wave celerity has been defined depending on the speed index to take into account flow regime transitions responsible for flow blockage.

In most cases HLLS and ARoe flux solvers reproduce identical results. The expected theoretical order of accuracy is effectively achieved by both numerical schemes. First order HLLS is in some cases more diffusive than the ARoe cases, but it is able to provide accurate numerical results in all cases, also in third order. The performance of the HLLS scheme turns out to be very satisfactory. 

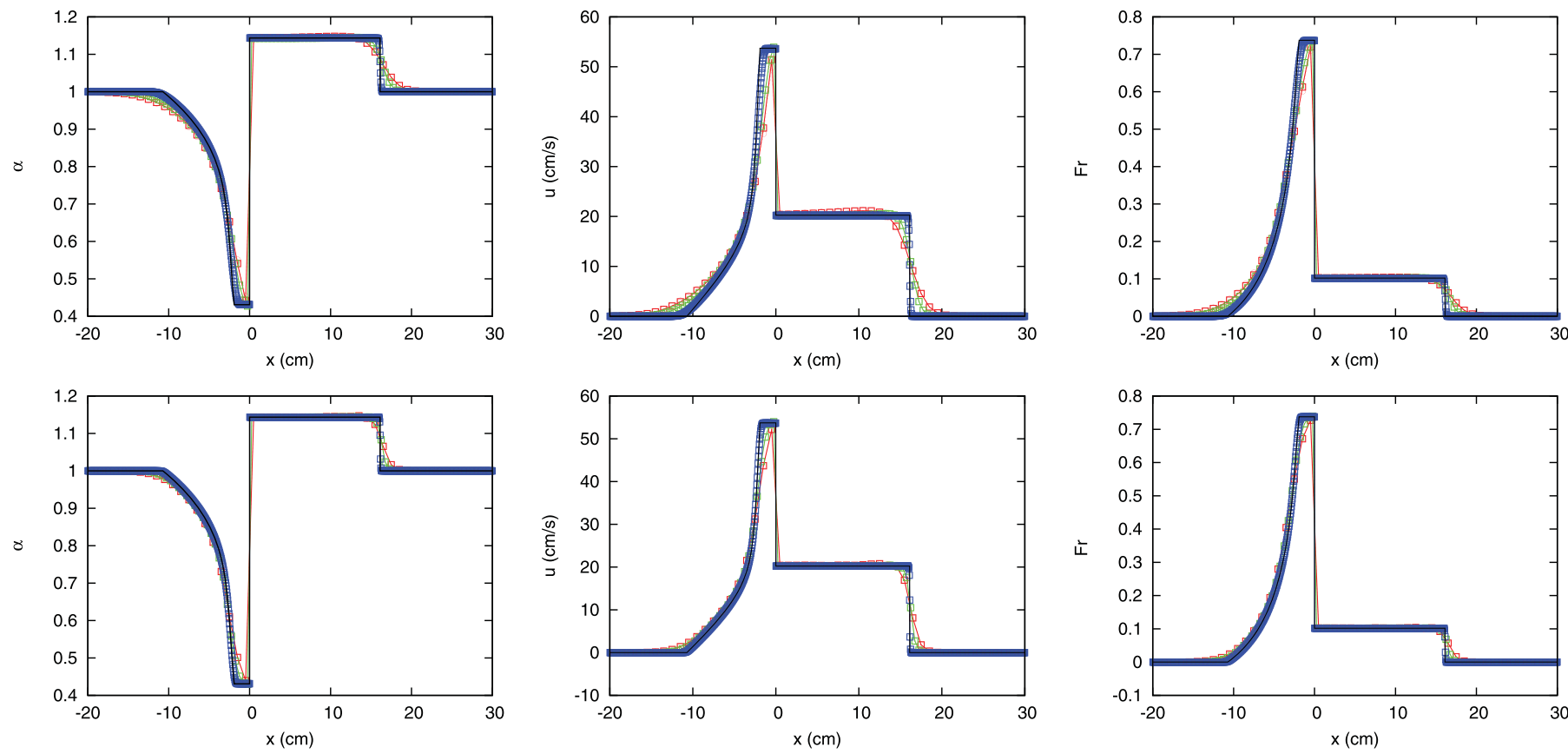

Fig. 19. Section 8.5. Test case 14. Subsonic RP in a vein. Comparison between exact (--) and numerical solutions at $t=0.1 \mathrm{~s}$ using the first order (upper) and TVDRK3 method (lower) using $\Delta x=1$ (red), 0.5 (green), 0.05 (blue) cm, for the HLLS solver - $\circ$ - and ARoe solver $-\square-$. (For interpretation of the references to colour in this figure legend, the reader is referred to the web version of this article.)
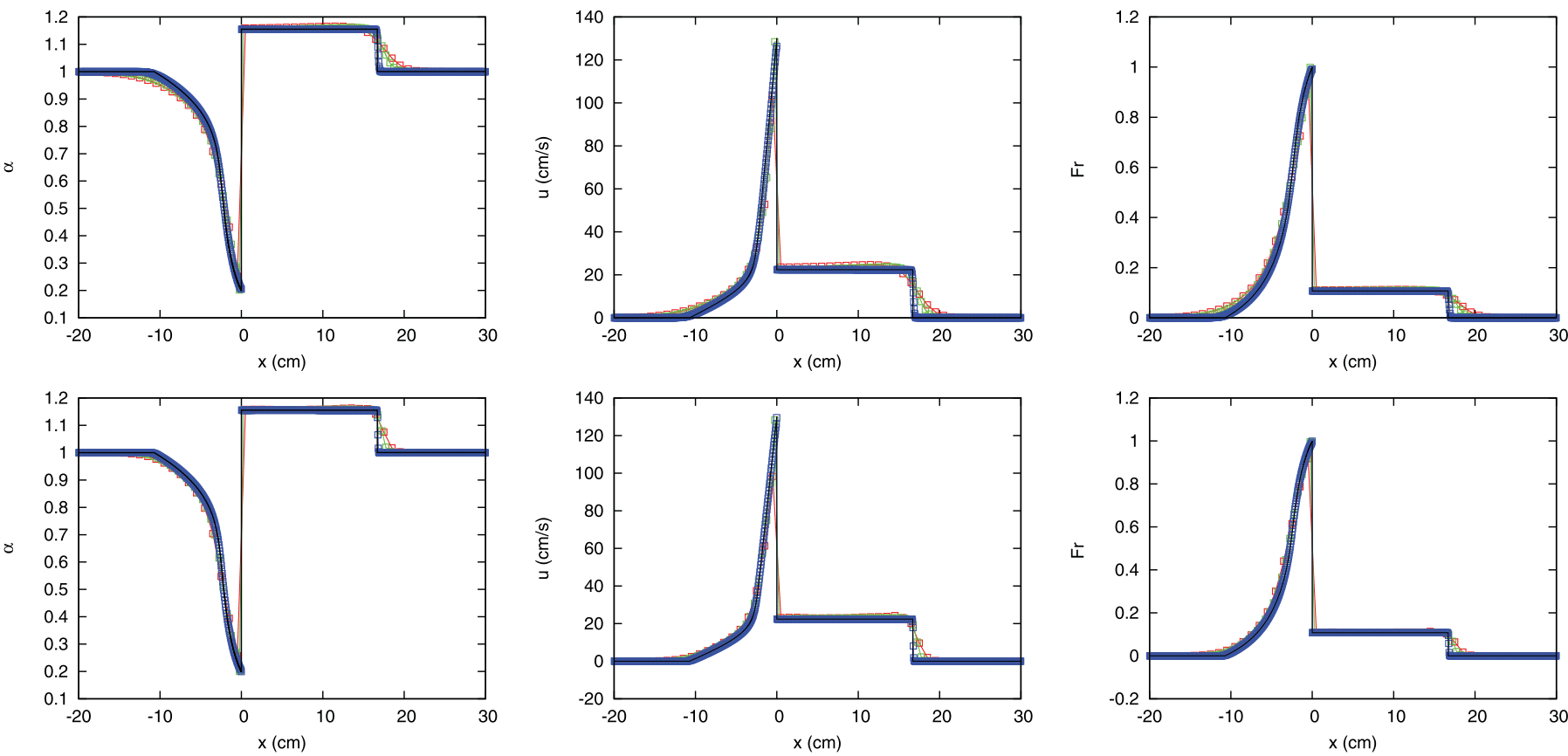

Fig. 20. Section 8.5. Test case 15. Sonic blockage in a vein. Comparison between exact (--) and numerical solutions at $t=0.1 \mathrm{~s}$ using the first order (upper) and TVDRK3 method (lower) using $\Delta x=1$ (red), 0.5 (green), 0.05 (blue) cm, for the HLLS solver - $\circ$ - and ARoe solver $-\square-$. (For interpretation of the references to colour in this figure legend, the reader is referred to the web version of this article.) 

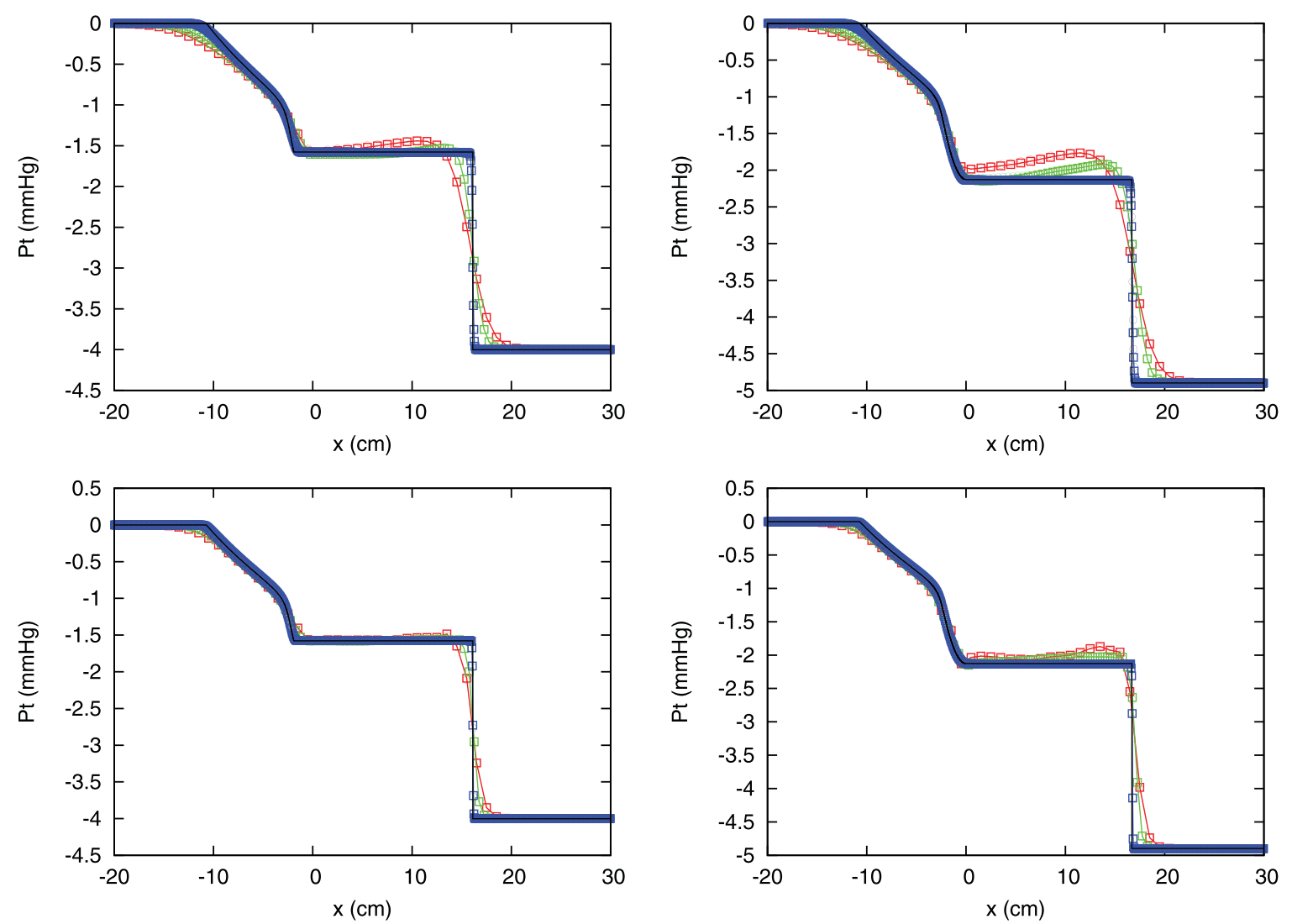

Fig. 21. Section 8.5. Comparison of results for total energy $P_{t}$ in test cases (left) 14 and (right) 15 . Comparison between exact (--) and numerical solutions at $t=0.1 \mathrm{~s}$ using the first order (upper) and TVDRK3 method (lower) using $\Delta x=1$ (red), 0.5 (green), 0.05 (blue) cm, for the HLLS solver $-\circ-$ and ARoe solver $-\square-$. (For interpretation of the references to colour in this figure legend, the reader is referred to the web version of this article.)
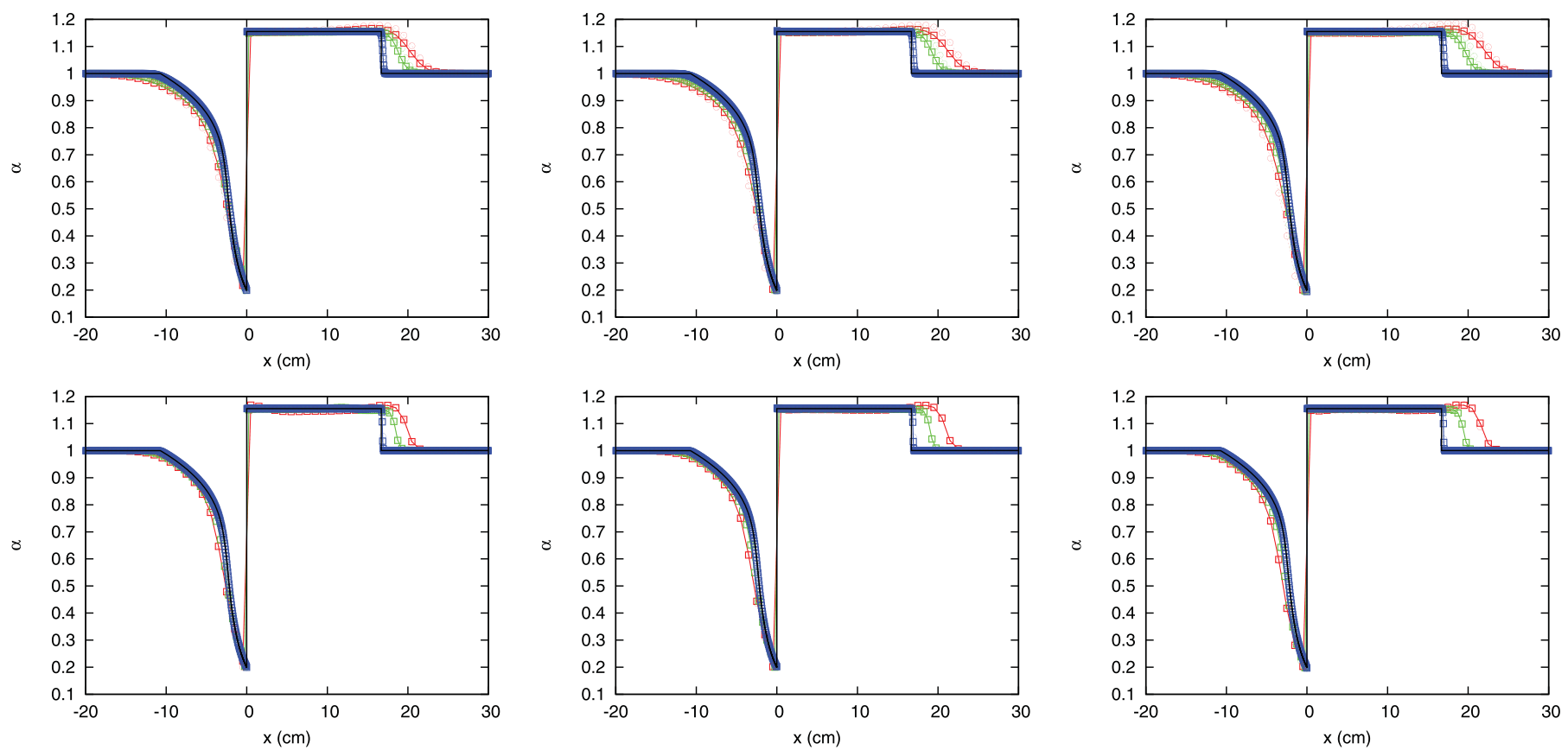

Fig. 22. Section 8.5. Test cases (left) 16 , (middle) 17 and (right) 18 . Sonic blockage in a vein. Comparison between exact (--) and numerical solutions at $t=0.1 \mathrm{~s}$ using the first order (upper) and TVDRK3 method (lower) using $\Delta x=1$ (red), 0.5 (green), 0.05 (blue) cm, for the HLLS solver $-\circ-$ and ARoe solver $-\square-$. (For interpretation of the references to colour in this figure legend, the reader is referred to the web version of this article.) 

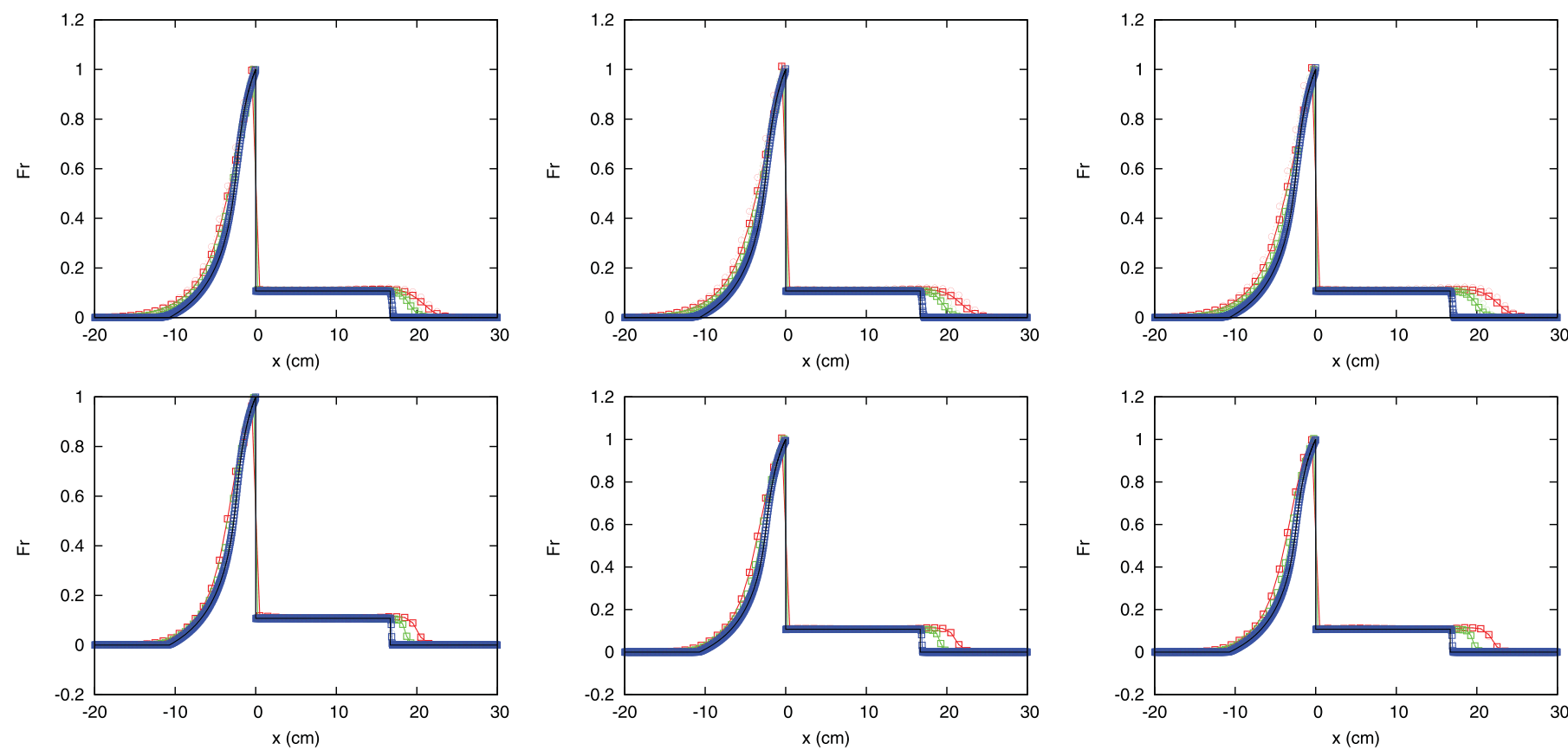

Fig. 23. Section 8.5. Test cases (left) 16 , (middle) 17 and (right) 18. Sonic blockage in a vein. Comparison between exact (--) and numerical solutions at $t=0.1 \mathrm{~s}$ using the first order (upper) and TVDRK3 method (lower) using $\Delta x=1$ (red), 0.5 (green), 0.05 (blue) cm, for the HLLS solver $-\circ-$ and ARoe solver $-\square-$. (For interpretation of the references to colour in this figure legend, the reader is referred to the web version of this article.)
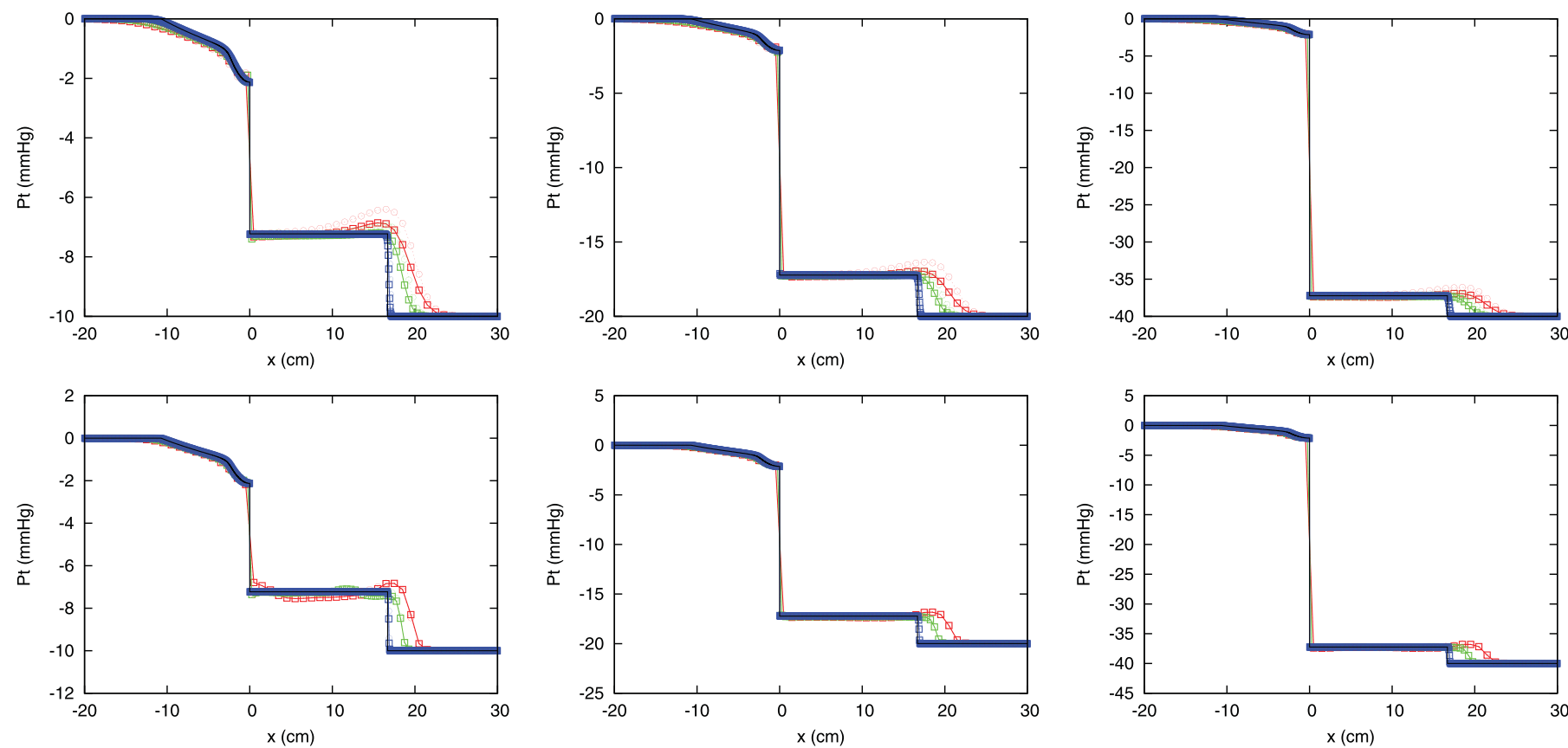

Fig. 24. Section 8.5. Test cases (left) 16, (middle) 17 and (right) 18. Sonic RP in a vein. Comparison between exact (--) and numerical solutions at $t=0.1 \mathrm{~s}$ using the first order (upper) and TVDRK3 method (lower) using $\Delta x=1$ (red), 0.5 (green), 0.05 (blue) cm, for the HLLS solver $-\circ-$ and ARoe solver $-\square-$. (For interpretation of the references to colour in this figure legend, the reader is referred to the web version of this article.)

\section{Acknowledgements.}

This project has been funded with support from the Dextera A.S. company. We would like to thank to Dr. José Manuel Ramírez Rodríguez for interesting discussions.

\section{Appendix A. Pulse wave velocity}

The values of $K(x)$ and $P_{o}(x)$ in (4) and the values of $A_{0}(x), m$ and $n$ in $\sigma$ are parameters that account for mechanical and ge- ometrical properties of the vessel. The parameter $P_{0}$ and $A_{0}$ are used to provide a reference point in the tube law, and the stiffness parameter $K$ is related to the deformability of the vessel. Typical values of coefficients $m$ and $n$ in arteries are $m=1 / 2$ and $n=0$ [5] while in the much more elastic veins these coefficients are $m=10$ and $n=-3 / 2[4,52]$, derived from the buckling behaviour of thin-walled vessels [53].

The definition of the approximate pulse wave velocity, $\widetilde{c}$ in (29) requires the computation of average values for $\widetilde{A^{m-1}}$ and $\widetilde{A^{n-1}}$ 
Jacobian are defined as smooth functions of the conserved variables in the left and right side of the Riemann Problem

lowing:

$\delta A^{q}=\widetilde{A^{q-1}} \delta A$,

with $q=m$ or $q=n$. The approximate average value $\widetilde{A^{q-1}}$ can be directly estimated as a simple quotient,

$\widetilde{A^{q-1}}=\left\{\begin{array}{cc}\frac{\delta A^{q}}{\delta A} & \text { if } \delta A>\epsilon \\ A A^{q-1} & \text { otherwise }\end{array}\right.$

where $\epsilon$ avoids division by zero.

One of the most advantageous characteristics of the Roe averages is that they decompose differences without requiring any limiting parameter as in (A.2). In this work, Roe averages for $\widetilde{A^{q-1}}$ in cases where $q$ is a rational number are presented:

- In case $q=0$, the average value in (A.1) is $\widetilde{A^{q-1}}=0$.

- If $q$ is a positive integer, average value is given by

$$
\widetilde{A^{q-1}}=\sum_{s=0}^{q-1} A_{i+1}^{q-1-s} A_{i}^{s} .
$$

- If $q$ is of the form $q=\frac{l}{2}$, with $l$ a positive integer, the average value is given by

$$
\widetilde{A^{q-1}}=\frac{\sum_{s=0}^{2 q-1} A_{i+1}^{\frac{1}{2}(2 q-1-s)} A_{i}^{\frac{s}{2}}}{\sqrt{A_{i+1}}+\sqrt{A_{i}}} .
$$

- If $q$ is of the form $q=-\frac{l}{2}$, with $l$ a positive integer, the average value is given by

$$
\widetilde{A^{q-1}}=-\frac{\sum_{s=0}^{-(2 q+1)} A_{i+1}^{\frac{(2 q+s)}{2}} A_{i}^{-\frac{s+1}{2}}}{\left(\sqrt{A_{i+1}}+\sqrt{A_{i}}\right)} .
$$

The average values $\widetilde{A_{o}^{m+1}}$ and $\widetilde{A_{0}^{n+1}}$ in (25) express pressure variations provided by variations in the reference cross-sectional area in $\widetilde{\sigma_{A_{0}}}$. Even if these parameters do not participate in the formulation of the pulse wave velocity, it may be of interest to consider their estimation. These average values must satisfy

$\delta A_{0}^{-q}=\widetilde{A_{0}^{-(q+1)}} \delta A_{0}$,

with $q=m$ or $q=n$, to recover exactly pressure variations in (21) Depending on the value of $q$, the average value $\widetilde{A_{0}^{-(q+1)}}$ is defined for the following cases of interest:

- If $q=0$, the average value is $\widetilde{A_{o}^{-(q+1)}}=0$.

- If $q$ is a positive integer, with $q>0$, the average value is

$$
\widetilde{A_{o}^{-(q+1)}}=-\sum_{s=0}^{q-1} A_{o, i+1}^{(-q+s)} A_{o, i}^{-(s+1)} \text {. }
$$

- If $q$ is of the form $q=\frac{l}{2}$, with $l$ a positive integer, the average value is given by

$$
\widetilde{A_{o}^{-(q+1)}}=-\frac{\sum_{s=0}^{2 q-1} A_{o, i+1}^{\frac{(-2 q+s)}{2}} A_{o, i}^{-\frac{(s+1)}{2}}}{\left(\sqrt{A_{o, i+1}}+\sqrt{A_{o, i}}\right)} .
$$

- If $q$ is of the form $q=-\frac{l}{2}$, with $l$ a positive integer, the average value is given by

$$
\widetilde{A_{o}^{-(q+1)}}=\frac{\sum_{s=0}^{-(2 q+1)} A_{o, i+1}^{\frac{-(2 q+1+s)}{2}} A_{o, i}^{\frac{s}{2}}}{\sqrt{A_{o, i+1}}+\sqrt{A_{o, i}}} .
$$

The definitions in (A.3), (A.4), and (A.5) inserted in (27) allow to restore pressure difference exactly, and the average values in the
${\widetilde{A^{m-1}}}_{i+\frac{1}{2}}=\widetilde{A^{m-1}}\left(A_{i+1}, A_{i}\right), \quad{\widetilde{A^{n-1}}}_{i+\frac{1}{2}}=\widetilde{A^{n-1}}\left(A_{i+1}, A_{i}\right)$. come

When setting $A_{i+1}=A_{i}$ the average values $\widetilde{A^{m-1}}$ and $\widetilde{A^{n-1}}$ be-

${\widetilde{A^{m-1}}}_{i+\frac{1}{2}}=m A_{i}^{m-1}, \quad{\widetilde{A^{n-1}}}_{i+\frac{1}{2}}=n A_{i}^{n-1}$,

that is,

$\left(\widetilde{\sigma_{A}}\right)_{i+\frac{1}{2}}=m A_{i}^{m-1}-n A_{i}^{n-1}$,

and in case that $K_{i+1}=K_{i}$, we find

$\widetilde{c}_{i+\frac{1}{2}}^{2}=\left(\frac{\mathcal{A}}{\rho} \bar{K} \widetilde{\sigma}_{A}\right)_{i+\frac{1}{2}}=K_{i} \frac{m A_{i}^{m}-n A_{i}^{n}}{\rho}=c_{i}^{2}=c_{i+1}^{2}$,

so the differential form of the Jacobian matrix is exactly recovered as demanded in Roe type Jacobians.

\section{References}

[1] Liang F, Liu H. Simulation of hemodynamic responses to the valsalva maneuver: an integrative computational model of the cardiovascular system and the autonomic nervous system. J Physiol Sci 2006;56:45-65.

[2] Anliker M, Rockwell RL, Odgen E. Nonlinear analysis of flow pulses and shock waves in arteries. Z ang Math Phys 1971;22:217-46.

[3] Pedley TJ. The fluid mechanics of large blood vessels. Cambridge: Cambridge University Press; 1980.

[4] Brook B, Falle S, Pedley T. Numerical solution for unsteady gravity-driven flows in collapsible tubes: evolution and roll-wave instability of a steady state. Fluid Mech 1999;396:223-56.

[5] Formaggia L, Lamponi D, Quarteroni A. One-dimensional models for blood flow in arteries. J Eng Math 2003;47:251-76.

[6] Sherwin S, Formaggia L, Peiró J, Franke V. Computational modelling of 1d blood flow with variable mechanical properties and its application to the simulation of wave propagation in the human arterial system. Int J Numer Meth Fluids 2003;43:673-700.

[7] Formaggia L, Lamponi D, Tuveri M, Veneziani A. Numerical modeling of $1 \mathrm{~d}$ arterial networks coupled with a lumped parameters description of the heart. Comput Method Biomec 2006;9:273-88.

[8] Kolachalama V, Bressloff N, Nair P, Shearman C. Predictive hemodynamics in a one dimensional human carotid artery bifurcation. IEEE Trans Biomed Eng 2007; $54: 802-12$

[9] Cavallini N, Caleffi V, Coscia V. Finite volumes and WENO scheme in one-dimensional vascular system modelling. Comput Math Appl 2008;56:2382-97.

[10] Formaggia L, Quarteroni A, Vergara C. On the physical consistency between three-dimensional and one-dimensional models in haemodynamics. J Comput Phys 2013;244:97-112.

[11] Delestre O, Lagrée P. A well-balanced finite volume scheme for blood flow simulation. Int J Numer Meth Fluids 2013;72. 177-05

[12] Köppl T, Wohlmuth1 B, Helmig R. Reduced one-dimensional modelling and numerical simulation for mass transport in fluids. Int J Numer Meth Fluids 2013;72:135-56.

[13] Xiu D, Sherwin S. Parametric uncertainty analysis of pulse wave propagation in a model of a human arterial network. J Comput Phys 2007;226:1385-407.

[14] Mynard J, Nithiarasu P. A 1d arterial blood flow model incorporating ventricular pressure, aortic valve and regional coronary flow using the locally conservative galerkin $(\mathrm{lcg})$ method. Commun Numer Meth Eng 2008:24:367-417.

[15] Marchandise E, Willemet M, Lacroix V. A numerical hemodynamic tool for predictive vascular surgery. Med Eng Phys 2009;31:131-44.

[16] Alastruey J, Khir A, Matthys K, Segers P, Sherwin SJ, Verdonck PR, Parker KH Peiró J. Pulse wave propagation in a model human arterial network: assessment of 1-d visco-elastic simulations against in vitro measurements. J Biomech $2011 ; 44: 2250-8$

[17] Toro EF, Siviglia A. Flow in collapsible tubes with discontinuous mechanica properties: mathematical model and exact solutions. Commun Comput Phys 2012;13:361-85.

[18] Toro EF, Siviglia A. Simplified blood flow model with discontinuous vessel properties: analysis and exact solutions. Modelling physiological flows series: modelling, simulation and applications. Ambrosi D, Quarteroni A, Rozza G, editors. Milan Dordrecht Heidelberg London New York: Springer-Verlag; 2012.

[19] Müller LO, Montecinos GI, Toro EF. Some issues in modelling venous haemodynamics. Numerical methods for hyperbolic equations: theory and applications An international conference to honour Professor. Toro EF, Vázquez-Cendón ME, Hidalgo A, García-Navarro P, Cea L, editors. Boca Raton London New York Leiden: CRC Press, Taylor \& Francis Group; 2013.

[20] Müller LO, Toro EF. Well-balanced high-order solver for blood flow in networks of vessels with variable properties. Int J Numer Method Biomed Eng 2013;29:1388-411

[21] Bermudez A, Vázquez-Cendón ME. Upwind methods for hyperbolic conservation laws with source terms. Comput Fluids 1994;23:1049-71. 
[22] Navas-Montilla A, Murillo J. Asymptotically and exactly energy balanced augmented flux-ADER schemes with application to hyperbolic conservation laws with geometric source terms. J Comput Phys 2016;317:108-47.

[23] Navas-Montilla A, Murillo J. Overcoming numerical shockwave anomalies using energy balanced numerical schemes. application to the shallow water equations with discontinuous topography. J Comput Phys 2017;340:575-616.

[24] Greenberg J, Leroux A. A well-balanced scheme for the numerical processing of source terms in hyperbolic equations. SIAM J Numer Anal 1996:33:1-16.

[25] LeFloch PG, Thanh MD. A godunov-type method for the shallow water equations with discontinuous topography in the resonant regime. J Comput Phys 2011;230:7631-60

[26] Siviglia A, Toffolon M. Multiple states for flow through a collapsible tube with discontinuities. J Fluid Mech 2014;761:105-22.

[27] Müller LO, Leugering G, Blanco PJ. Consistent treatment of viscoelastic effects at junctions in one-dimensional blood flow models. J Comput Phys 2016;314:167-93.

[28] Hou T, LeFloch P. Why nonconservative schemes converge to wrong solutions: error analysis. Math Comp 1994;62:497-530.

[29] LeFloch PG, Mishra S. Numerical methods with controlled dissipation for small-scale dependent shocks. Acta Numer 2014;23:1-72.

[30] Huang PG, Muller LO. Simulation of one-dimensional blood flow in networks of human vessels using a novel TVD scheme. Appl Math Comput 2015;31:e02701.

[31] Spiller C, Toro EF, Vázquez-Cendón ME, Contarino C. On the exact solution of the Riemann problem for blood flow in human veins, including collapse. Appl Math Comput 2017;303:178-89.

[32] Xing Y, Shu CW. A survey of high order schemes for the shallow water equations. J Math Study 2014;47:221-49.

[33] Murillo J, García-Navarro P. Weak solutions for partial differential equations with source terms: application to the shallow water equations. J Comput Phys 2010;229:4327-68.

[34] Murillo J, García-Navarro P. Energy balance numerical schemes for shallow water equations with discontinuous topography. J Comput Phys 2013;236:119-42.

[35] Murillo J, García-Navarro P. Accurate numerical modeling of 1d flow in channels with arbitrary shape. application of the energy balanced property. J Comput Phys 2014;260:222-48.

[36] Murillo J, Navas-Montilla A. A comprehensive explanation and exercise of the source terms in hyperbolic systems using roe type solutions. Appl. 1D-2D Shallow Water Equs.Adv. Water Resour. 2016;98:70-96.

[37] Murillo J, García-Navarro P. Augmented versions of the HLL and HLLC riemann solvers including source terms in one and two dimensions for shallow flow applications. J Comput Phys 2012;231:6861-906.

[38] Murillo J, García-Navarro P. A roe type energy balanced solver for $1 \mathrm{~d}$ arterial blood flow and transport. Comput. Fluids 2015;117:149-67.

[39] Navas-Montilla A, Murillo J. Energy balanced numerical schemes with very high order. the augmented roe flux ADER scheme. application to the shallow water equations. J Comput Phys 2015;290:188-218.

[40] Caleffi V, Valiani A. Well balancing of the SWE schemes for moving-water steady flows. J Comput Phys 2017;342:85-116.
[41] Müller LO, Parés C, Toro EF. Well-balanced high-order numerical schemes for one-dimensional blood flow in vessels with varying mechanical properties. J Comput Phys 2013;242. 53-5

[42] Burguete J, García-Navarro P, Murillo J. Friction term discretization and limitation to preserve stability and conservation in the 1d shallow-water model: application to unsteady irrigation and river flow. Int J Numer Meth Fluids 2008;58:403-25.

[43] Murillo J, García-Navarro P, Burguete J. Time step restrictions for well-balanced shallow water solutions in non-zero velocity steady states. Int J Numer Meth Fluids 2009;60:1351-77.

[44] Murillo J, García-Navarro P. Wave riemann description of friction terms in unsteady shallow flows: application to water and mud/debris floods. J Comput Phys 2012:231:1963-2001.

[45] Müller LO, Toro EF. A global multiscale mathematical model for the human circulation with emphasis on the venous system. Int J Numer Method Biomed Eng 2014;30:681-725.

[46] LeVeque RJ. Balancing source terms and flux gradients in high-resolution godunov methods: the quasi-steady wave-propagation algorithm. J Comput Phys 1998;146:346-65.

[47] Leveque R. Finite volume methods for hyperbolic problem. New York: Cambridge University Press; 2002.

[48] Toro EF. Riemann solvers and numerical methods for fluid dynamics: a practical introduction. third. Berlin, Heidelberg: Springer-Verlag; 2009.

[49] Harten A, Hyman JM. Self adjusting grid methods for one-dimensional hyperbolic conservation laws. J Comput Phys 1983;50:235-69.

[50] Davis SF. Simplified second-order godunov-type methods. SIAM J Sci Stat Comput 1988;9:445-73.

[51] Einfeldt B. On godunov-type methods for gas dynamics. SIAM J Numer Anal $1988 ; 25: 294-318$.

[52] Shapiro AH. Steady flow in collapsible tubes. J Biomech Eng 1977;99:126-47.

[53] Flaherty JE, Keller JB, Rubinow SI. Post buckling behavior of elastic tubes and rings with opposite sides in contact. SIAM J Appl Math 1972;23:446-55.

[54] Gottlieb S, Shu CW. Total variation diminishing runge-kutta schemes. Math Comput 1998;67(221):73-85.

[55] Jiang GS, Shu CW. Efficient implementation of weighted ENO schemes. J Comput Phys 1996;126. 202-28

[56] Dumbser M, Enaux C, Toro EF. Finite volume schemes of very high order of accuracy for stiff hyperbolic balance laws. J Comput Phys 2008;227:3971-4001.

[57] Xing Y. High order finite volume WENO schemes for the shallow water flows through channels with irregular geometry. J Comput Appl Math 2016;299. 3229-244

[58] de Vahl Davis G. Natural convection of air in a square cavity a bench mark numerical solution. Int J Num Meth Fluids 1983;3:249-64.

[59] Farrell PA, Hegarty AF, Miller JJH, O'Riordan E, Shishkin GI. Robust computational techniques for boundary layers. Boca Raton, FL: Chapman \& Hall/CRC: 2000.

[60] Navas-Montilla A, Murillo J. 2D well-balanced augmented ADER schemes for the shallow water equations with bed elevation and extension to the rotating frame. J Comput Phys 2018;372:316-48. 